\title{
Lignin phenol formaldehyde resins synthesised using South African spent pulping liquor.
}

by

Chelaine Maree

Thesis presented in partial fulfilment of the requirements for the Degree

of

MASTER OF ENGINEERING (CHEMICAL ENGINEERING)

in the Faculty of Engineering at Stellenbosch University

Supervisor

Prof JF Görgens

Co-Supervisor

Dr L Tyhoda

December 2021 


\section{DECLARATION}

By submitting this thesis electronically, I declare that the entirety of the work contained therein is my own, original work, that I am the sole author thereof (save to the extent explicitly otherwise stated), that reproduction and publication thereof by Stellenbosch University will not infringe any third-party rights and that I have not previously in its entirety or in part submitted it for obtaining any qualification.

Date: December 2021 


\section{Abstract}

The current study investigated to which extent phenol could be replaced by lignins to produce lignin phenol formaldehyde (LPF) resins. This was done utilising soda lignin and sodium lignosulphonate as byproducts from the South African pulping industry.

These industrial lignins were characterised for phenolic hydroxyl group content, proximate analyses, midinfrared (IR) spectroscopy, and thermogravimetric analyses (TGA). Soda black liquor was either spraydried or acid precipitated to isolate the soda lignin. The acid precipitated soda lignin was then further acid purified. The lignosulphonate was received in spray-dried form and purified using a dialysis membrane of $3500 \mathrm{Da}$. Purified soda lignin indicated the highest reactivity towards formaldehyde and was therefore investigated further to produce LPF resins at $60 \%, 80 \%$ and $100 \%$ substitution of phenol by lignin. The LPF resins were characterised for their free formaldehyde content, $\mathrm{pH}$ values, mid-IR spectroscopy, thermogravimetric analyses, and shear strength.

At $60 \%$ and $80 \%$ substitution a one-pot phenolation and LPF synthesis method was used where the soda lignin was first phenolated before formaldehyde was added, while at $100 \%$ substitution no phenolation was performed. Phenolation increased the phenolic hydroxyl content by up to $105 \%$ and $124 \%$ at $80 \%$ and $60 \%$ substitution, respectively. Three face-centred central composite designs (CCD) were used to attain the maximum adhesive strength at each substitution level. The parameters optimised in each CCD included the phenolation reaction temperature, sodium hydroxide to phenolated lignin ( $\mathrm{NaOH} / \mathrm{PL}$ ) ratio, and formaldehyde to phenolated lignin (F/PL) ratio. The best synthesis reaction conditions at $60 \%$ and $80 \%$ substitution were at the lower bound of the range tested, with a $\mathrm{NaOH} / \mathrm{PL}$ molar ratio of 0.124 and a F/PL molar ratio of 1 . At $100 \%$ substitution without any modification, the optimum $\mathrm{NaOH} / \mathrm{PL}$ molar ratio was 0.477 , while the best F/PL molar ratio was at 3 , which was the upper bound of the tested range.

Plywood made with LPF60, LPF80, and LPF100 resins attained their highest shear strengths of $0.786,1.09$, and $0.987 \mathrm{MPa}$, respectively, which adhered to the GB/T 14732-2013 standard ( $\geq 0.7 \mathrm{MPa}$ ). A substitution level of $68 \%$ produced the highest shear strength of $1.11 \mathrm{MPa}$. The good performance of this soda lignin as a LPF resin was further confirmed by producing high-density particleboard with the LPF68 resin giving a MOR and MOE of $40 \mathrm{MPa}$ and $3209 \mathrm{MPa}$, respectively, which adhered to the ANSI A208.1-1999 standard. Furthermore, good resistance of the resin to water absorption (37.2 wt\%) and thickness swelling (13.5 wt\%) was also proven. This unclean pulping lignin was the first lignin to produce LPF resins at $100 \%$ substitution, without requiring modification or additives. 


\section{Opsomming}

Hierdie studie het gemik om die maksimum vervanging van fenol met lignien te beriek, om lignien fenol formaldehied (LPF) harpuis te produseer. Dit was gedoen deur twee ligniene te ondersoek: ' $n$ soda lignien van ' $n$ soda pulpproses met suikerriet, en ' $n$ lignosulfaat van $n$ natriumsulfiet pulpproses met Eukaliptus grandis, om as by-produkte van die Suid-Afrikaanse pulpindustrie benut te word. Hierdie industriële ligniene was gekarakteriseer in terme van strukturele, komposisionele en termiese eienskappe. Die lignienryke afvalstroom van die soda pulpproses was of gedroogspuit, of gepresipiteer deur swaelsuur te doseer, om die lignien te isoleer. Nadat die lignien gepresipiteer was, was dit ook gesuiwer deur swaelsuur te gebruik. Die lignosulfonaat was gesuiwer deur 'n 3500 Da dialisemembraan. Vanuit die karakterisering het die gesuiwerde soda lignien die meeste potensiaal getoon as ' $n$ fenol plaasvervanger, dus was die lignien verder gebruik om LPF harpuis te vervaardig by vervanging van $60 \%, 80 \%$ en $100 \%$ van fenol met lignien. Die LPF harpuis was ook verder gekarakteriseer vir vrye formaldehied, $\mathrm{pH}$ waardes, strukturele eienskappe, termiese eienskaappe, en skuifsterkte.

By $60 \%$ en $80 \%$ vervanging, was ' $n$ kontinue proses van eers fenolasie met die soda lignien, en daarna harpuis-sintese uitgevoer, terwyl by $100 \%$ vervangin daar geen fenolasie toegepas was nie. Fenolasie het die aantal fenoliese hidroksiede vermeerder tot en met $105 \%$ en $124 \%$ by vervanging van $60 \%$ en $80 \%$ onderskeidelik. Drie sentraal-saamgestelde-ontwerpe was gebruik om die skuifsterkte van die harpuis te maksimeer by elke persentasie vervanging. Drie veranderlikes was geoptimeer: die temperatuur waarby fenol met lignien reageer; die verhouding van natriumhidroksied tot fenol en lignien ( $\mathrm{NaOH} / \mathrm{PL}$ ); en die verhouding van formaldehied tot fenol en lignien ( $F / P L)$. Die beste sintese reaksiekondisies in die getoetste omvang by $60 \%$ en $80 \%$ vervanging was by ' $n \mathrm{NaOH} / \mathrm{PL}$ molêre verhouding van 0.124 , en ' $n$ F/PL molêre verhouding van 1 . By $100 \%$ vervanging sonder fenolasie modifikasie, was die beste sintese reaksiekondisies by ' $\mathrm{n} \mathrm{NaOH} / \mathrm{PL}$ molêre verhouding van 0.477 , terwyl die beste F/PL molêre verhouding die boonste grens van die getoetsde verhouding by 3 was.

Laaghout gemaak deur die verskillende LPF harpuis by $60 \%, 80 \%$, en $100 \%$ te gebruik, het die hoogste skuifsterkte van $0.786,1.09$, en $0.987 \mathrm{MPa}$, respektiewelik by elke persentasie vervanging bereik, wat voldoen het aan die GB/T 14732-2013 laaghout standaard ( $\geq 0.7 \mathrm{MPa}$ ). Die hoogste skuifsterkte bereik was 1.11 MPa na fenolasie van gesuiwerde soda lignien en LPF sintese by ' $n 68 \%$ vervanging. Die goeie potensiaal van die soda lignien vir LPF harpuis sintese was verder bevestig deur hoe digtheid partikelbord te produseer by $68 \%$ vervanging. ' $n$ Modulus van skeuring en elastisiteit van $23.5 \mathrm{MPa}$ en $2750 \mathrm{MPa}$ was onderskeidelik geproduseer, wat voldoen het aan die ANSI A208.1-1999 standaard. Verder het die harpuis ook goeie weerstand teen water absorpsie en dikte swelling getoon, met 37.2 massa \% en 13.5 massa \%, onderskeidelik. Dus toon hierdie soda lignien baie potensiaal om as ' $n$ fenol vervanger in LPF harpuis gebruik te word in houttoepassings, sonder om enige modifikasie van die lignien, of bymiddels tydens die termiese verhardingsproses te benodig. 


\section{Acknowledgements}

This research was financially sponsored by the Paper Manufacturers Association of South Africa (PAMSA), with Sappi Ltd being the industry partner. The conclusions, findings, opinions, and recommendations made are that of the author and are not necessarily that of the sponsors.

The author would like to honour the following people for their continued support:

- Prof Görgens for the opportunity to do this study, and for his guidance and wisdom throughout

- Dr Tyhoda (Dept of Forestry and Wood Sciences) for his guidance and wisdom also

- The abovementioned sponsors for the opportunity to pursue a master's degree at Stellenbosch University

- Dr Nelson Sefara and Dr Sanet Minnaar (Sappi Technology Centre) for their support

- Mr Solomon and Mr Hendrikse (Dept of Forestry and Wood Sciences) for their assistance and friendliness

- Mr Alvin Petersen and the rest of the technical staff at the Dept of Process Engineering for their assistance

- For my family and friends for all their love and support

- My Lord and Saviour Jesus Christ, for His unending love and strength 


\section{Table of contents}

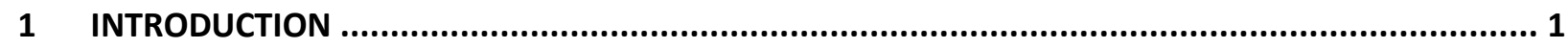

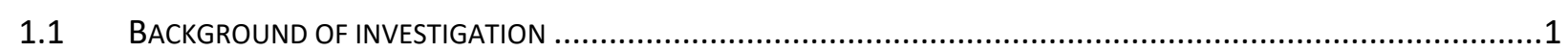

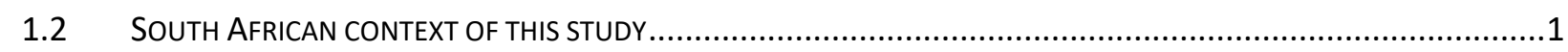

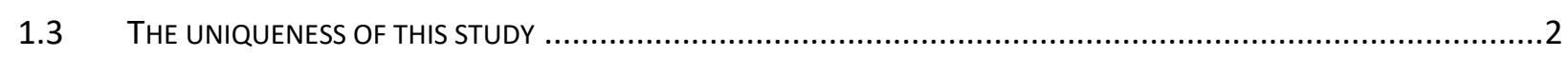

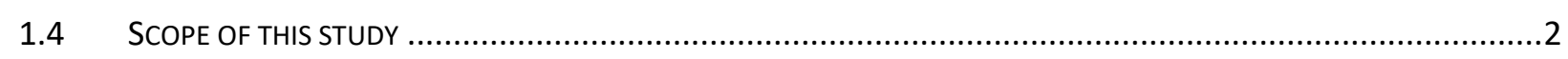

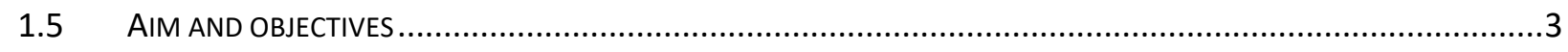

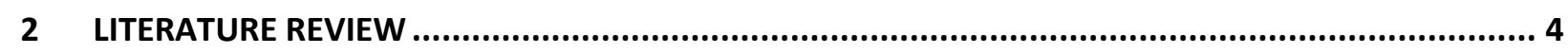

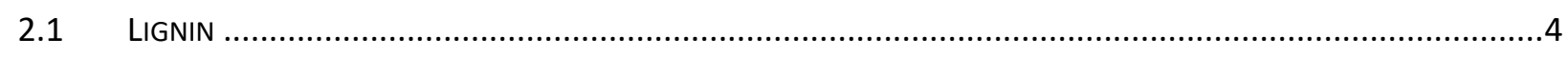

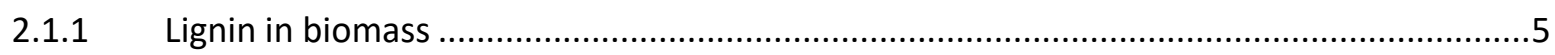

2.1.2 Effect of pulping processes on lignin structure .........................................................

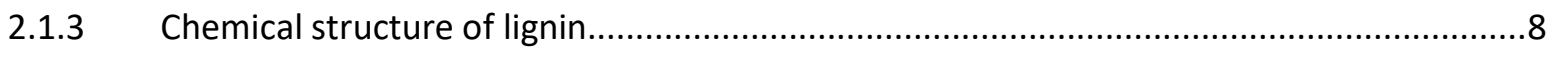

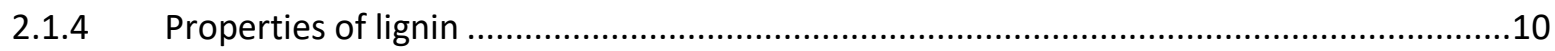

2.1.5 Hydrophilic nature of lignosulphonates ....................................................................12

2.1.6 Acid precipitation and purification of lignin ...................................................................12

2.1.7 Membrane purification of lignosulphonates.............................................................13

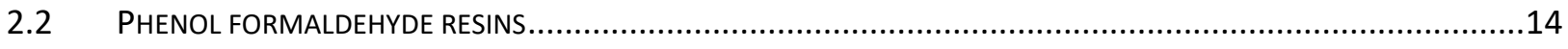

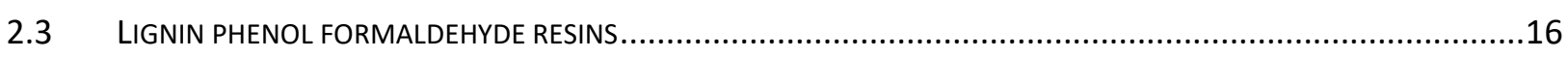

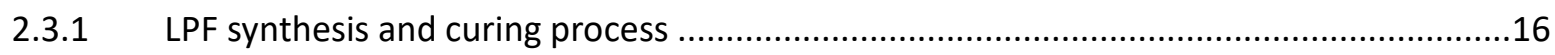

2.3.2 Effect of LPF synthesis and curing parameters on the resin performance .......................18

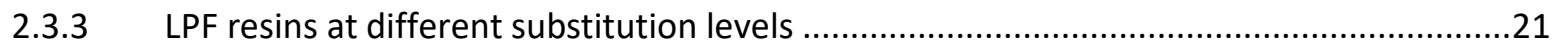

2.4 CHEMICAL MODIFICATION METHODS TO ENHANCE LIGNIN REACTIVITY .................................................2

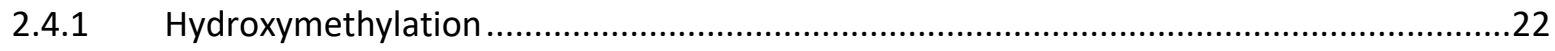

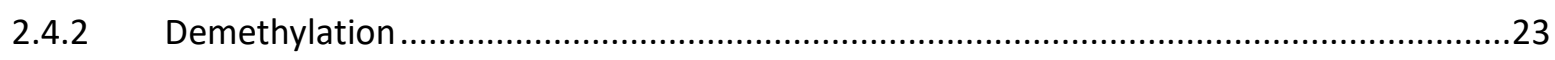

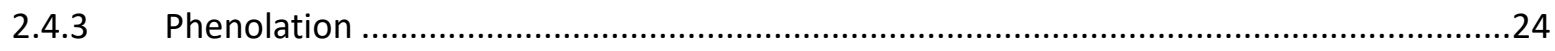

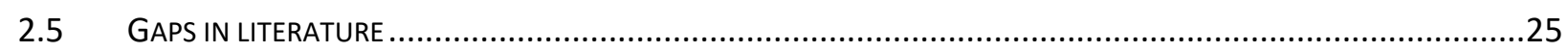

3 MATERIALS AND METHODOLOGY

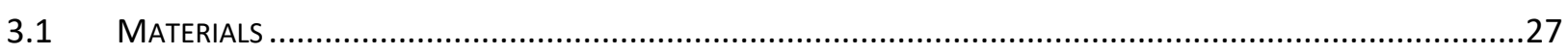

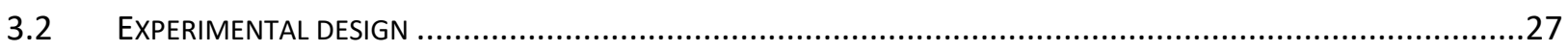

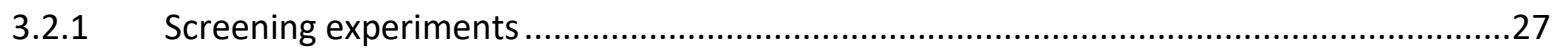

3.2.2 Face-centred central composite design .....................................................................28

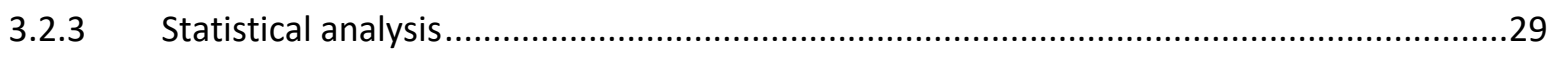

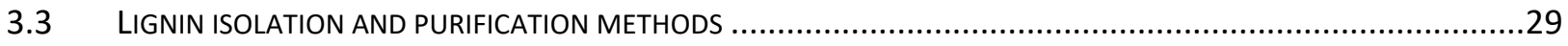




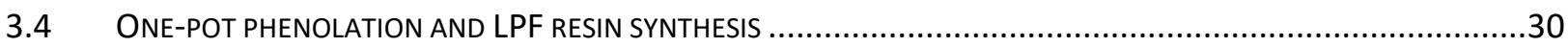

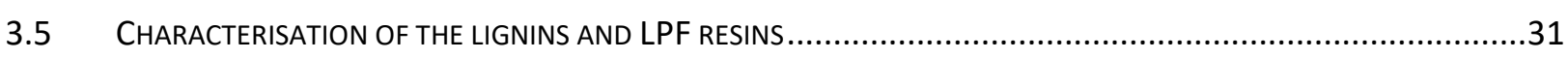

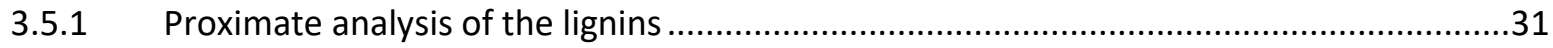

3.5.2 Phenolic hydroxyl content determination of the lignins and LPF resins .........................32

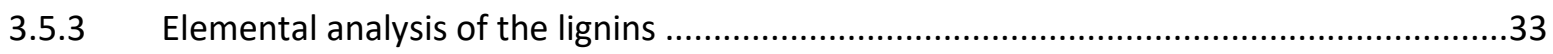

3.5.4 Thermogravimetric analysis of the lignins and LPF resins.........................................3

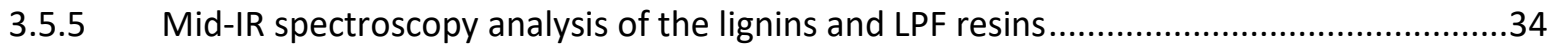

3.5.6 Free formaldehyde content determination of the LPF resins ....................................34

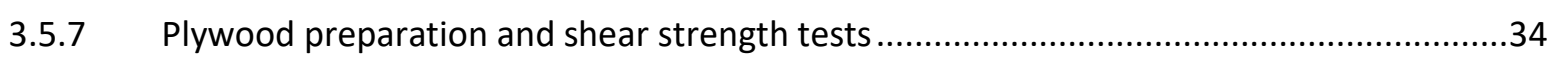

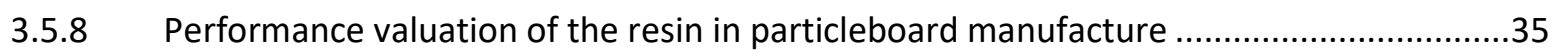

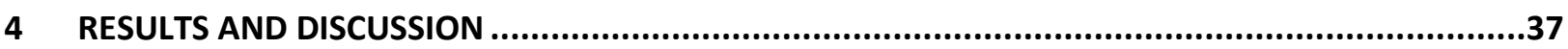

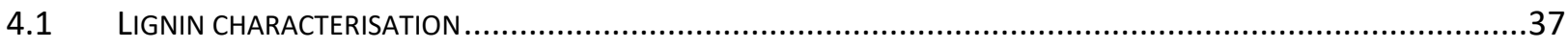

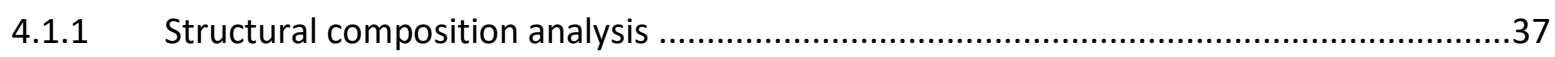

4.1.2 Mid-IR spectra of the lignin and lignosulphonate samples .......................................38

4.1.3 TGA results of the lignin and lignosulphonate samples .............................................42

4.1.4 Screening of shear strength results of LPF resins made from soda lignin and lignosulphonate ......................................................................................................44

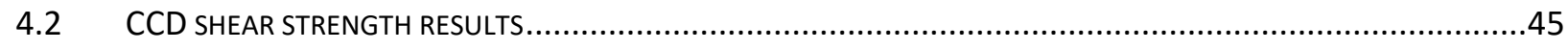

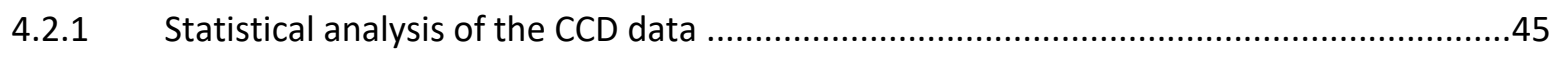

4.2.2 The effect of phenolation modification on the lignin characteristics ...........................49

4.2.3 The effect of the phenolation reaction temperature on the shear strength .....................51

4.2.4 The effect of the $\mathrm{NaOH} / \mathrm{PL}$ and $\mathrm{F} / \mathrm{PL}$ molar ratios on the shear strength ......................52

4.2.5 Optimum substitution level and reaction conditions............................................56

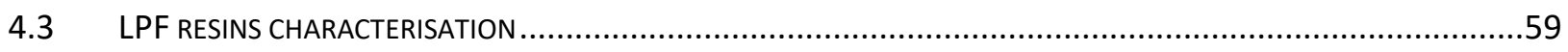

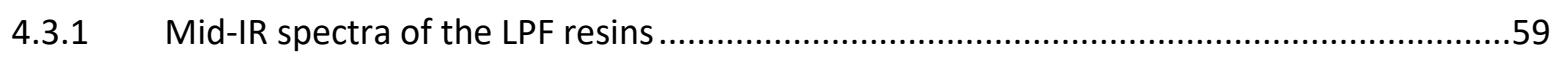

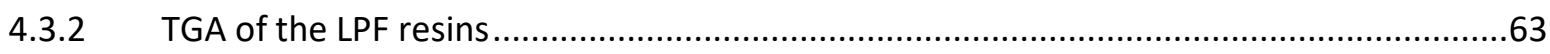

4.3.3 Free formaldehyde content and $\mathrm{pH}$ of the LPF resins...........................................67

4.3.4 Performance valuation of the resin in particleboard manufacture ..................................68

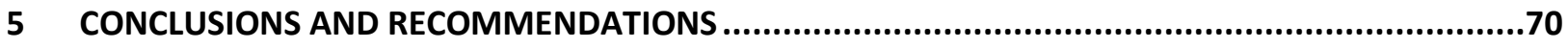

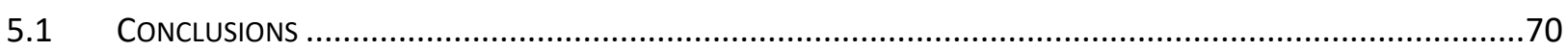

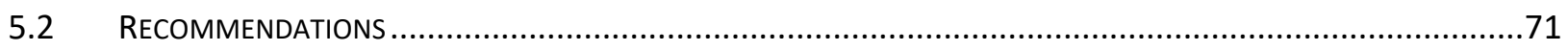

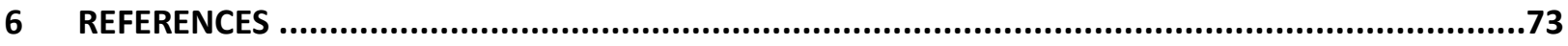


1 ADDENDUM A - COMPARISON OF DIFFERENT PHENOLATION AND SYNTHESIS REACTION

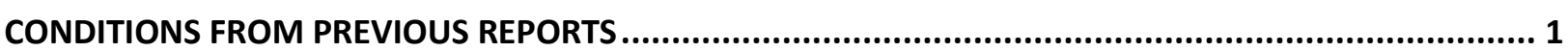

2 ADDENDUM B - SHEAR STRENGTH RESULTS OF THE LPF RESINS …...................................... 4

3 ADDENDUM C - MID-IR SPECTRA OF THE LPF RESINS ....................................................... 5 


\section{List of figures}

Figure 1. Structures of the three monolignols. Redrawn from Pfungen (2015).

Figure 2. Reaction paths for the addition of formaldehyde to phenol. Redrawn from Tonge (2007)...... 15 Figure 3. Methylolation reaction between formaldehyde and a G-unit of lignin. Reproduced from (Hemmilä et al., 2017).

Figure 4. Representation of the reactive sites on phenol and the lignin units. Reproduced from Hemmilä, et al. (2017).

Figure 5. Addition of phenol onto the G-unit of lignin. Reproduced from Jiang et al. (2018)................ 24

Figure 6. Experimental setup for one-pot phenolation and LPF synthesis. ............................................ 31

Figure 7. Calibration curve for converting concentration of phenolic hydroxyl groups to absorbance. .. 33 Figure 8. Mid-IR spectra of the different lignin samples in the single (4000 to $2500 \mathrm{~cm}^{-1}$ ), triple (2500 to

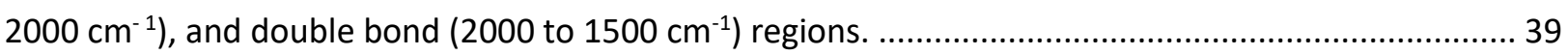

Figure 9: Mid-IR spectra of the different lignin samples in the fingerprint region $\left(1500\right.$ to $\left.600 \mathrm{~cm}^{-1}\right) \ldots . .39$ Figure 10. Scree plot of the eigenvalues of the principal components and their percentage contributions

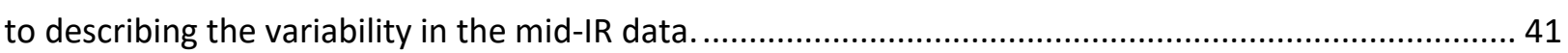

Figure 11. PCA plots obtained from the mid-IR spectra of the lignin and lignosulphonate samples. ...... 42

Figure 12. TGA and DTG graphs of the soda lignin and lignosulphonate samples.

Figure 13. Comparison between the shear strengths of the lignosulphonate and soda lignin LPF100 resins made at centre point conditions.

Figure 14. Profiles for predicted values and desirability of the CCD shear strength results of the LPF100 resins. The green line represents the predicted curve, the red lines represent optimums and the blue lines the upper and lower bounds.

Figure 15. Profiles for predicted values and desirability of the CCD shear strength results of the LPF80 resins. The green line represents the predicted curve, the red lines represent optimums and the blue lines the upper and lower bounds.

Figure 16. Profiles for predicted values and desirability of the CCD shear strength results of the LPF60 resins. The green line represents the predicted curve, the red lines represent optimums and the blue lines the upper and lower bounds.

Figure 17. The mid-IR spectra of the purified soda lignin before and after phenolation. 51

Figure 18. Contour plots of the effect of the $\mathrm{NaOH} / \mathrm{PL}$ ratio and phenolation reaction temperature on the shear strength (MPa). 52

Figure 19. Contour plots of the effects of $\mathrm{F} / \mathrm{PL}$ and $\mathrm{NaOH} / \mathrm{PL}$ molar ratios on the shear strength (MPa) at different substitution levels.

Figure 20. Shear strengths of the S-A-P LPF100, LPF80, and LPF60 resins made at different reaction conditions, as determined by the experimental CCDs.

Figure 21. Desirability profile of the combined effects of the substitution level (from $60 \%$ to $100 \%$ ), the $\mathrm{NaOH} / \mathrm{PL}$ ratio, and the F/PL ratios. The green line represents the predicted curve, the red lines represent optimums and the blue lines the upper and lower bounds. 57

Figure 22. Scores plot of PC1 and PC2 from the mid-IR data of the different LPF resins. 61 
Figure 23. Scatter plot correlating the methylene bridges formed at $1470 \mathrm{~cm}^{-1}$ from the mid-IR data to the shear strength of the LPF resins.

Figure 24. TGA and DTG graphs of the LPF100_(0,0) resins synthesised with S-A-P, L-S and L-S-D. 63

Figure 25. TGA and DTG graphs of the S-A-P LPF resins that produced the highest shear strength at each substitution level. 64

Figure 26. TGA and DTG graphs of the LPF100 resins made with S-A-P lignin phenolated and synthesised at different reaction conditions.

Figure 27. TGA and DTG graphs of the LPF80 resins made with S-A-P lignin phenolated and synthesised at different reaction conditions 66

Figure 28. TGA and DTG graphs of the LPF60 resins made with S-A-P lignin phenolated and synthesised at different reaction conditions. 67

Figure 29. Mid-IR spectra of the LPF100 lignosulphonate resins at centre point conditions. 5

Figure 30. Mid-IR spectra of the S-A-P LPF100 resins of the centre point and highest shear samples. ..... 6

Figure 31. Mid-IR spectra of the S-A-P LPF100 resins with low shear strengths.

Figure 32. Mid-IR spectra of the S-A-P LPF80 resins of the centre point, highest shear, and lowest shear samples.

Figure 33. Mid-IR spectra of the S-A-P LPF60 resins of the centre point and highest shear samples. 9

Figure 34. Mid-IR spectra of the S-A-P LPF60 resins with low shear strengths 10

Figure 35. Mid-IR spectra of the S-A and S-A-P LPF68 resins at optimum conditions. 11 


\section{List of tables}

Table 1. Composition of cellulose, hemicellulose, and lignin in different lignocellulosic biomass sources.

Table 2. Average contribution of the principal phenolic building blocks in lignin for different biomass sources. (Gellerstedt et al., 2009).

Table 3. Properties of technical lignins (Lora, 2008; Laurichesse \& Avérous, 2014; Ruwoldt, 2020)....... 10

Table 4. Sample IDs for different lignin samples that were used.

Table 5. The three-factor, three-level CCD used to maximise the shear strength of the LPF60 and LPF80 LPF resins

Table 6. The two-factor, three-level CCD used to maximise the shear strength of the LPF100 resins..... 29 Table 7: The phenolation temperatures, $\mathrm{NaOH} / \mathrm{PL}$ ratios, and F/PL ratios used in the CCD to maximise the shear strength at each substitution level.

Table 8. Compositions of lignin samples in terms of moisture, ash, organic, and phenolic $\mathrm{OH}$ contents (Standard deviations reported in brackets).

Table 9. Elemental analysis results of the lignin samples. 38

Table 10: Peak assignments for the mid-IR spectra as from previous reports. 40

Table 11. ANOVA results of the CCD shear strengths optimisation of the LPF100 resins. 46

Table 12. ANOVA results of the CCD shear strengths optimisation of the LPF80 resins 47

Table 13. ANOVA results of the CCD shear strength optimisation of the LPF60 resins. 48 Table 14. Phenolic hydroxyl content of the phenolated lignin samples at $80 \%$ and $60 \%$ substitution, at different $\mathrm{NaOH} / \mathrm{PL}$ molar ratios and phenolation temperatures.

Table 15. ANOVA of the combined effects of substitution level, $\mathrm{NaOH} / \mathrm{PL}$ ratio, and F/PL ratio of all three CCDs.

Table 16. ANOVA of the effects from the substitution level $(60 \%$ to $80 \%), \mathrm{NaOH} / \mathrm{PL}$ ratio, and $\mathrm{F} / \mathrm{PL}$ ratio on the shear strength. 58

Table 17. Mid-IR spectra band assignments of the LPF resins. 59 Table 18. Free formaldehyde content, shear strength and $\mathrm{pH}$ values of the centre point and optimum LPF resin samples. 68

Table 19. Moisture content (MC), thickness swelling (TS), water absorption (WA), modulus of rupture (MOR), and modulus of elasticity (MOE) of the particleboard produced with LPF68 and Bondtite (Standard deviations reported in brackets). 68

Table 20. Different reaction parameters of phenolation in alkaline conditions as used in previous reports.

Table 21. Different reaction parameters of phenolation in acidic conditions as used in previous reports.1 Table 22. Different LPF synthesis parameters as used in previous reports. .......................................... 2 Table 23. Shear strength results from the CCD runs with the S-A-P LPF100, LPF80 and LPF60 resins....... 4 


\section{NOMENCLATURE}

\begin{tabular}{|c|c|}
\hline \multicolumn{2}{|c|}{ Acronyms } \\
\hline Al & Aliphatic \\
\hline ANOVA & Analysis of variance \\
\hline $\operatorname{Ar}$ & Aromatic \\
\hline A.Q. & Anthraquinone \\
\hline $\mathrm{CCD}$ & Central composite design \\
\hline DTG & Differential thermogravimetry \\
\hline FC & Folin-Ciocalteu \\
\hline IR & Infrared \\
\hline LF & Lignin formaldehyde \\
\hline LPF & Lignin phenol formaldehyde \\
\hline MC & Moisture content \\
\hline MOE & Modulus of elasticity \\
\hline MOR & Modulus of rupture \\
\hline MWCO & Molecular weight cut-off \\
\hline NREL & National Renewable Energy Laboratory \\
\hline NSSC & Neutral sulphite semi-chemical \\
\hline PAMSA & Paper Manufacturers Association of South Africa \\
\hline PC & Principal component \\
\hline PCA & Principal component analysis \\
\hline PDI & Polydispersity Index \\
\hline $\mathrm{PF}$ & Phenol formaldehyde \\
\hline TGA & Thermogravimetric analysis \\
\hline TS & Thickness swelling \\
\hline WA & Water absorption \\
\hline \multicolumn{2}{|l|}{ Symbols } \\
\hline $\mathrm{F}$ & Formaldehyde \\
\hline $\mathrm{F} / \mathrm{PL}$ & Formaldehyde to phenolated lignin \\
\hline $\mathrm{M}_{\mathrm{w}}$ & Molecular weight \\
\hline$M_{n}$ & Number average of molecular weight \\
\hline $\mathrm{NaOH} / \mathrm{PL}$ & Sodium hydroxide to phenolated lignin \\
\hline$P$ & Phenol \\
\hline$T_{d}$ & Thermal decomposition temperature \\
\hline $\mathrm{T}_{\mathrm{g}}$ & Glass transition temperature \\
\hline
\end{tabular}




\section{INTRODUCTION}

\subsection{Background of investigation}

A global striving to use fewer fossil fuels has motivated the total or partial replacement of petroleum-derived phenol (P) by lignin to produce lignin phenol formaldehyde (LPF) resins. This study investigates the utilisation of lignin from the South African pulp industry to replace phenol in LPF resins for wood applications.

PF resins are thermosetting polymers commonly used as wood adhesives in plywood and other wood composite constructions. Phenolic compounds are derived from the petroleum, petrochemical, coal, or phenol-producing industries. Phenol can therefore be subject to price fluctuations and is limited by crude oil reserves. Furthermore, it is toxic, carcinogenic and could lead to mutagenic or teratogenic defects. Therefore, it is considered a pollutant and is environmentally unsustainable (Calace et al., 2002).

Lignin is the second most abundant biopolymer on earth and exists in plant biomass. Lignin is considered a renewable source as it is obtained from plants, which are renewable if managed sustainably (Belgacem \& Gandini, 2008). Lignin naturally has a very complex structure and could be described as heterogeneous polyphenols with large molecular masses (Belgacem \& Gandini, 2008). The pulping industry uses hardwoods, softwoods, or annual plant species to isolate cellulose fibres, which are used to produce packaging, paper, tissues, and other products. Lignin is separated from these fibres during the pulping process, where it is solubilised and then removed in a by-product stream termed black liquor or spent pulping liquor. Currently, lignin is mostly used as a fuel for combustion to produce heat and energy (Bajwa et al., 2019). The underutilisation of lignin as high-value by-products (for example in adhesives, pesticides, fertilisers, biomaterials) from pulping processes is largely due to the complex structure of lignin, which is influenced by the biomass source, pulping process, and isolation processes used to extract it (Sixta, 2006). Lignin could potentially also be more cost-effective than phenol, as it is available as a commercial by-product that could be utilised for high-value applications. Therefore, LPF resins can potentially produce a greener, bio-based adhesive than conventional PF resins as lignin is not toxic and renewable.

\subsection{South African context of this study}

The South African pulping industry strive to be economically, environmentally, and socially responsible. The Paper Manufacturers Association of South Africa (PAMSA) is involved with a lot of research to improve waste valorisation in the pulp and paper industries of South Africa, and they represent more than $90 \%$ of the paper, packaging, and tissue manufacturers of South Africa. In South Africa, lignin is available as a by-product suspended in a black liquor stream from the pulp and paper industry. Most of the black liquor is currently combusted in units for energy recovery or cooking chemicals recovery, which leads to reduced energy and chemical requirements. Excess of black liquor produced could however cause bottlenecks in the energy recovery plants, requiring black liquor to be stored which could cause a decrease in its heating value and overall quality (Namane, Sithole \& Ramjugernath, 2015). Therefore, it is desired to find a high-value application for utilising lignin from these black liquors, which could be more economically and environmentally favourable than combustion for energy and could reduce bottlenecks in energy recovery plants. In this study, two locally available lignins provided by Sappi Ltd were investigated. These two lignins were obtained from different 
pulping processes and biomass sources: from soda pulping with sugarcane bagasse from the Stanger mill, and sodium sulphite pulping with Eucalyptus Grandis from the Tugela mill.

\subsection{The uniqueness of this study}

The lignins used in this study are unique in their structure and characteristics, as they are dependent on the biomass sources, pulping methods and isolation methods used. Therefore, their characteristics and behaviour as an LPF resin cannot be predicted based on previous reports from other lignins, thus an investigation was required to determine the properties of these lignins and their potential to replace phenol. Several studies have investigated the substitution of phenol by lignin for wood applications (Lee, Chang \& Tseng, in press; Sarkar \& Adhikari, 2000; Abdelwahab \& Nassar, 2011; Zhang et al., 2013; Ghorbani et al., 2016; Ghorbani, Konnerth, et al., 2018). However, only two studies (Kalami et al., 2017, 2018; Govender, Majeke, et al., 2020) have succeeded in replacing phenol to produce lignin formaldehyde (LF) resins at a hundred percent substitution. However, additives were incorporated during the adhesive formulation to be able to achieve acceptable properties for plywood applications. In the study by Govender et al. (2020), six different technical lignins from the South Africa pulping industry were tested to produce LF resins. The results were promising. However, the bonding strength obtained was lower than required by industrial plywood standard GB/T 147322013 if no additives were used. With the addition of hexamine as a cross-linker and glyoxal or epichlorohydrin as hardeners, plywood could be produced adhering to the standard. Kalami et al. $(2017,2018)$ were able to produce LF resins (using Kraft, soda, sulphite, organosolv, and enzymatic hydrolysis lignins) which adhered to the standard. However, wheat flour was used as an additive and alder bark (modal) was used as a filler. $\mathrm{NaOH}$ was also required to dissolve the resins during the adhesive formulation. Yaakob, Bin Roslan, Salim \& Zakaria (2021) also produced LF resins recording a shear strength of $1.76 \mathrm{MPa}$ when tested according to the JIS K-6852 standard. The lignin used was produced from empty fruit bunch using sulfuric acid and glacial acetic acid. It was not reported how the adhesive was formulated.

\subsection{Scope of this study}

This study aimed to produce a LPF resin at $100 \%$ substitution without any additives required for use in wood adhesives. Furthermore, the study also investigated enhancing the reactivity of the lignin at $60 \%$ and $80 \%$ substitution of phenol by lignin on a weight percentage basis. A soda black liquor was received, there the soda lignin was isolated using acid precipitation and acid purification with sulphuric acid. Another soda lignin sample was received in a spray-dried form and used without treatment. The lignosulphonate was received as a spraydried powder and purified using a dialysis membrane. The lignins were characterised by proximate analysis, elemental analysis, mid-infrared (mid-IR) spectroscopy, thermogravimetric analysis (TGA), and determination of phenolic hydroxyl content. Screening experiments were then done with the purified soda lignin and with lignosulphonate before and after dialysis, at $100 \%$ substitution. It was concluded from the lignin characterisations and screening experiments that the soda lignin that was acid precipitated and acid purified, showed the most promise as a phenol replacement and was therefore further investigated.

A one-pot method (Zhao et al., 2016) was used for the first time with a pulping lignin, where the phenol and lignin were reacted before LPF synthesis which increased the number of reactive sites and also cleaved 
methoxyl groups, thereby reducing steric hindrance. Thus, this lignin-phenol solution would have a higher reactivity towards formaldehyde. The acid purified soda lignin and phenol were reacted in alkaline conditions at either $60 \mathrm{wt} \%$ or $80 \mathrm{wt} \%$ lignin with the residual $40 \mathrm{wt} \%$ and $20 \mathrm{wt} \%$ phenol, respectively, to form a ligninphenol solution. Thereafter, formaldehyde was added to start the synthesis reaction. Thus, the phenol was added to the lignin in a manner that will achieve the $60 \%$ or $80 \%$ substitution in the lignin-phenol solution, hence no more lignin or phenol was added later.

Three face-centred CCDs were used to find the maximum shear strengths obtainable at $60 \%, 80 \%$ and $100 \%$ substitution. The F/PL molar ratio and $\mathrm{NaOH} / \mathrm{PL}$ molar ratio was optimised at each substitution level, and the phenolation temperature was also optimised at $60 \%$ and $80 \%$ substitution. The resins were characterised for free formaldehyde content, pH values, mid-IR spectroscopy, and TGA. The LPF resins were then tested for use as adhesives in plywood and particleboard applications.

\subsection{Aim and objectives}

This study aimed to utilise technical lignins from the South African pulping industry to replace phenol in LPF resin production.

- Isolation and purification of lignins (using methods published in previous reports)

- Characterisation of isolated lignins

- Performance evaluation of unmodified isolated technical lignins

- Performance evaluation of modified isolated technical lignins to improve their reactivity

- Synthesis of LPF resins

- Optimising some synthesis parameters

- Characterisation of resins produced with special focus on bonding strength, curing characteristics, and free formaldehyde content 


\section{LITERATURE REVIEW}

\subsection{Lignin}

Lignin is the most abundant native aromatic biopolymer on earth and makes up $15 \%$ to $35 \%$ of the cell wall in plants (Gan \& Pan, 2019). There are three main components of lignocellulosic biomass and they are lignin, cellulose and hemicellulose (Hu et al., 2011). Lignin occurs mainly in the plant cell middle lamella and the secondary wall (Sixta, 2006; Karunarathna \& Smith, 2020). It is structured as a very complex three-dimensional phenolic polymer with an undefined bulk structure and no repeating multiunit. The biomass species, environmental conditions, and the location of specific cells in the plant could alter the chemical composition, bonds formed, and structure of the lignin present in the plant (Chen, 2014). Technical lignin refers to lignin that has been derived from the delignification or processing of lignocellulosic biomass, such as in the pulping industry (Ekielski \& Mishra, 2021). The annual global production of technical lignins is approximately 100 million tons, mostly produced by the paper and pulp industry. Only approximately 1.65 million tons are valorised for commercial production, other than bioenergy generation. Of these globally produced commercially utilised lignins, lignosulphonates make up around $79 \%$, with Kraft lignin at around $16 \%$, and hydrolysis and soda lignin combined at around 5\% thereof (Dessbesell et al., 2020). Furthermore, lignin obtained as a by-product from the pulping industry also has its structure changed by the pulping method as well as the isolation method used to extract it from the by-product stream.

The utilisation of lignin is therefore complex on an industrial scale, due to the variability in the lignin properties and its complex polymeric structure, which causes steric hindrance and low reactivity (Henriksson et al., 2010). Most technical lignins are currently combusted for energy or heat recovery, as lignin is carbonaceous and can be used as a low-grade fuel (Bajwa et al., 2019). The pulping industry has shown great interest in the valorisation of lignin, which could be motivated by portfolio diversification, revenue generation, improving process efficiency and reducing production costs (Dessbesell et al., 2020). A current commercialised high-value application of lignosulphonates is the addition thereof to concrete for water reduction, improved strength development and workability (Wool \& Sun, 2005). Other commercialised examples include valorisation as dispersants, release controllers, resins, and binders (Dessbesell et al., 2020). Other potential high-value applications could include utilisation as activated carbons, carbon fibres, fertilisers, heavy metal adsorbents, hydrocarbons, pesticide carriers, phenolic compounds, and syngas (Ludmila et al., 2015; Bajwa et al., 2019). Lignin may also be used as a sole carbon source to culture bacteria and metabolise triglyceride lipids (Chio, Sain \& Qin, 2019). Biocompatible and non-toxic lignin has been used in nanomaterials and biomedical applications recently (Ekielski \& Mishra, 2021). Utilising lignin as a by-product is attractive, as it is polymeric, renewable, and sustainable and it may also contribute to waste valorisation. Lignin's phenolic structure makes it especially promising as a phenol replacement, for example in producing LPF resins. Although lignin aromatic structure could be valuable for utilisation in producing fuels and chemicals, current industrial processes from which lignin could be derived (for example the paper and pulp industry) are not optimised based on the utilisation of lignin as a by-product. Thus, there is a demand for more economically and environmentally sustainable methods to extract and utilise lignin on a commercial scale. 


\subsubsection{Lignin in biomass}

Native lignin is a polymer with a high molecular weight (from 6700 to 23500 for milled wood lignin from different biomasses (Tolbert et al., 2014)), and it is insoluble in water due to network formation with other biomass, such as cellulose and hemicellulose. Native lignin refers to the original structure, as present in the lignocellulose which has not been modified. It exists in all terrestrial plants and may also be present in some aquatic organisms (Lu \& Ralph, 2010; Vanholme et al., 2010). The main functions of lignin in plants include providing rigidity to cell walls, binding different woody tissues together, rendering cell walls hydrophobic, and protecting the wood against microbial degradation (Wool \& Sun, 2005; Henriksson et al., 2010; Fatehi \& Chen, 2016; Tian et al., 2016). Cellulose, hemicellulose and lignin are the main constituents in plants, with their compositions varying in different biomass materials (Laurichesse \& Avérous, 2014). Other minor constituents could include pectin, fats, wax, moisture and water-soluble compounds (Wool \& Sun, 2005), which are usually more abundant in grasses than in wood materials (Figoli, Alfredo \& Basile, 2016). Table 1 shows the difference in compositions of the lignocellulosic materials in different biomass sources. Softwoods generally contain more lignin ( $25 \mathrm{wt} \%$ to $35 \mathrm{wt} \%$ ) than hardwoods (18 wt\% to $25 \mathrm{wt} \%$ ), while grasses may contain a broader range (around 4 wt\% to $30 w t \%$ ) of lignin (Howard et al., 2003). However, the lignin content in plants may be influenced by climate and other factors as well, and any chemical, enzymatic, or mechanical action applied may influence the physical properties of lignin (Chen, 2014).

Table 1. Composition of cellulose, hemicellulose, and lignin in different lignocellulosic biomass sources.

\begin{tabular}{|c|c|c|c|c|c|c|}
\hline \multicolumn{2}{|c|}{ Lignocellulosic material } & \multirow{2}{*}{$\begin{array}{c}\begin{array}{c}\text { Cellulose } \\
\text { (wt\%) }\end{array} \\
46\end{array}$} & \multirow{2}{*}{$\begin{array}{c}\begin{array}{c}\text { Hemicellulose } \\
\text { (wt\%) }\end{array} \\
27\end{array}$} & \multirow{2}{*}{$\begin{array}{c}\begin{array}{c}\text { Lignin } \\
\text { (wt\%) }\end{array} \\
23\end{array}$} & \multirow{2}{*}{$\begin{array}{c}\begin{array}{c}\text { Other } \\
\text { (wt\%) }\end{array} \\
4\end{array}$} & \multirow{2}{*}{\begin{tabular}{l}
\multicolumn{1}{c}{ Reference } \\
(Alonso Pippo et al., \\
2011 )
\end{tabular}} \\
\hline & $\begin{array}{l}\text { Sugarcane } \\
\text { bagasse }\end{array}$ & & & & & \\
\hline \multirow[t]{3}{*}{ Grasses } & Wheat straw & 38.2 & 24.0 & 23.4 & 14.4 & \multirow{3}{*}{$\begin{array}{l}\text { (Maruthamuthu, } \\
\text { 2017) }\end{array}$} \\
\hline & Rice straw & 34.2 & 24.5 & 11.9 & 29.4 & \\
\hline & Corn stover & 35.6 & 22.1 & 12.3 & 30 & \\
\hline \multirow{3}{*}{ Hardwood } & Birch & 38.2 & 19.7 & 22.8 & 19.3 & \multirow{3}{*}{$\begin{array}{l}\text { (Maruthamuthu, } \\
\text { 2017) } \\
\text { (Howard et al., } \\
\text { 2003) }\end{array}$} \\
\hline & Willow & 43.0 & 29.3 & 24.2 & 3.5 & \\
\hline & Stems & 40 to 55 & 24 to 40 & 18 to 25 & & \\
\hline \multirow{3}{*}{ Softwood } & Spruce & 43.4 & 18.0 & 28.1 & 10.5 & \multirow{3}{*}{$\begin{array}{l}\text { (Maruthamuthu, } \\
\text { 2017) } \\
\text { (Howard et al., } \\
\text { 2003) }\end{array}$} \\
\hline & Pine & 46.4 & 22.9 & 29.5 & 1.2 & \\
\hline & Stems & 45 to 50 & 25 to 35 & 25 to 35 & & \\
\hline
\end{tabular}

\subsubsection{Effect of pulping processes on lignin structure}

Various pulping technologies are implemented on an industrial scale internationally. They aim to break the linkages between lignin, hemicellulose, and cellulose so that the hemicellulose and lignin will dissolve, and the remaining cellulose fibres could be recovered for paper and pulp production. Cellulose fibres could be 
chemically or mechanically separated from the biomass to obtain this key desired component in the paper and pulp industry. Pulping technologies can be categorised into four main categories: chemical, semi-chemical, chemi-mechanical, and mechanical pulping. The advantages of more chemical pulping are that fibres remain intact and are not broken during pulping producing higher purity and higher quality pulp. However, the chemicals could degrade hemicellulose and lignin in the pulp, which produces lower pulp yields than with mechanical pulping. Biomasses used for pulp production are hardwoods, softwoods and some grass species such as sugarcane bagasse (Gellerstedt, Ek \& Henriksson, 2009).

Black liquor is the spent cooking liquor obtained when the wood pulp is cooked in a digester. Cellulose fibres are released from the wood pulp in the digester and undergo further processing to produce pulp. The black liquor that remains is then an aqueous solution of lignin, hemicellulose, and inorganic pulping chemicals. More than half of the energy content fed into the wood digester usually ends up in the black liquor, rendering it an energy-rich stream (Annual integrated report, 2019). Black liquor is obtained as a by-product from the pulping process and due to its high lignin content, technical lignin could be isolated from it for high-value applications. Kraft, soda and sulphite pulping are the main industrial pulping processes that currently have the potential of producing technical lignins as by-products (Lu et al., 2017).

\subsubsection{Kraft pulping}

Kraft pulping uses sodium hydroxide $(\mathrm{NaOH})$, sodium sulphide $\left(\mathrm{Na}_{2} \mathrm{~S}\right)$ and hot water between 155 and $175{ }^{\circ} \mathrm{C}$. During Kraft pulping, the hydroxide and hydrosulphide anions react with the lignin, which then forms smaller alkali- or water-soluble fragments. The major fragmentation reaction involves the cleavage of the phenolic $\beta$ O-4 bonds by a nucleophilic attack of the hydrogen sulphide ion on the lignin, forming a quinone methide. (Gierer, 1980; Fearon, Kuitunen \& Vuorinen, 2016). $\mathrm{HO}^{-}, \mathrm{HS}^{-}$, and $\mathrm{S}^{2-}$ are common nucleophiles that attack the electron-deficient centres of lignin molecules and lead to the dissolution of lignin in alkaline solutions (Sixta, 2006). $\beta$-aryl ether linkages also get cleaved when a hydroxyl group is present on $C_{\alpha}$ (Demuner, I. F., Colodette, J. L., Demuner, A. J., and Jardim, 2019). The black liquor generated could contain sugars and inorganics along with lignin (Ekielski \& Mishra, 2021).

\subsubsection{Soda pulping}

Soda pulping was the first established chemical pulping process, developed in 1851 in England by Burges and Watts, whereafter the first soda pulping mill was established in the United States in 1866 (Biermann, 1996). An aqueous solution of sodium hydroxide is used as a cooking agent and the process operates at a pH value between eleven and thirteen and a temperature between 150 and $170{ }^{\circ} \mathrm{C}$ (Macfarlane, Mai \& Kadla, 2014). The soda process has a low selectivity, as under these conditions both lignin and carbohydrates could be degraded. Therefore, the process is performed traditionally with easily pulped materials such as hardwoods, flax, bagasse and straw (Laurichesse \& Avérous, 2014). Non-wood fibre is known to have a more accessible structure and lower lignin content compared to woody fibre, which allows lower pulping temperatures to be used (Lora, 2008). The soda pulping method is advantageous as it is a sulphur-free process, and it produces soda lignin with a low degree of impurities and also a low ash and sugar content (Lora, 2008). The resulting soda lignin also has low molecular weights, because of the cleavage of some of the linkages during soda pulping, and it is insoluble in water. The absence of sulphur as in lignin from soda pulping could be 
advantageous over lignosulphonate or kraft lignin that has sulphur-containing groups, as these could be volatile and toxic and renders the lignin hydrophilic, which may not be desired depending on the application (Chen, 2014). Cellulose could be degraded during pulping at too harsh conditions, therefore anthraquinone (A.Q.) is sometimes added to limit cellulose degradation, where the process is then termed soda-AQ pulping. During the soda pulping process, lignin may be depolymerised, lignin-carbohydrate bonds may be cleaved, and lignin-lignin condensation could occur, resulting in more available reactive sites. This depolymerisation increases the solubility of the lignin in alkaline solutions (Lora, 2008), it involves the hydrolysis of most of the $\beta-0-4$ bonds and may also include the partial modification or elimination of the aliphatic side chains (Gellerstedt et al., 2009). When these non-phenolic $\beta-0-4$ bonds are cleaved, phenolic lignin moieties and an epoxide are formed that may react further in extreme alkaline conditions (Ekielski \& Mishra, 2021).

\subsubsection{Sulphite pulping}

During sulphite pulping an aqueous solution of sulphite or bisulphite salt containing a base of ammonia, calcium, sodium, or magnesium, is used to digest the biomass. The solubility of sulphite salt depends on the $\mathrm{pH}$, therefore $\mathrm{Ca}^{2+}, \mathrm{Mg}^{2+}, \mathrm{Na}^{+}$, or $\mathrm{NH}_{4}{ }^{+}$could be used as a cation. Calcium precipitates at $\mathrm{pH} \geq 3$ and could be used in acidic bisulphite pulping, while magnesium could be used at $\mathrm{pH} \leq 5$. At a higher $\mathrm{pH}$ value, ammonium and sodium could be used as cations (Fan \& Zhan, 2008). The cation used affects the properties of the lignosulphonate (sulphonated lignin) produced, for example, sodium cations form more linear lignins, while calcium cations result in a compact lignosulphonate (Doherty, Mousavioun \& Fellows, 2011). Pressures of 500 to $700 \mathrm{kPa}$ are used along with temperatures around 120 to $150{ }^{\circ} \mathrm{C}$, while a wide $\mathrm{pH}$ range could be used as it varies depending on the solubility and dissociation characteristics of the salt used (Biermann, 1996). The sulphite process could be acidic ( $\mathrm{pH}$ of 1 to 5), or neutral sulphite semi-chemical ( $\mathrm{pH}$ of 5 to 7 ). The annual production of technical lignosulphonate has been reported to be around 1.8 million tons, which is the most dominant technical lignin produced annually (Aro \& Fatehi, 2017; Ruwoldt, 2020). The primary components of spent sulphite liquor formed includes lignosulphonate, acetic acid, hemicellulose sugars, inorganics, and derivatives of sugar dehydration. Some of these impurities, especially carbohydrates (such as hemicellulose sugars and some pulping chemicals), could be solubilised together with lignin to some extent. Advantages of sulphite pulping include high pulp yields, easy bleaching and refining of the pulp. Disadvantages of sulphite pulping include the formation of weaker pulps, not all species of wood could be easily processed, long cooking cycles are required, and cooking chemicals recovery is difficult or impossible.

The main reactions in sulphite pulping involving lignin include the formation of an $\mathrm{C}_{\alpha}$ cation by cleaving $\alpha-0-4$ ether bonds on the lignin, forming resonance stabilised benzylic cations. Sulphonation then occurs where a sulphonate group attaches to the $C_{\alpha}$ which renders the lignosulphonate soluble. The $\alpha$-sulphonic group has an electron-withdrawing effect on the $\beta-0-4$ group, which aids as sulphite attaches to the $C_{\beta}$ and sulphitolytic cleavage of the $\beta$-aryl-ether bond occurs (Ekielski \& Mishra, 2021). The resulting lignosulphonate is very complex due to the several reactions that could occur with the highly reactive sulphite and bisulphite species present, which forms various ions. The lignosulphonates are soluble over a wide $\mathrm{pH}$ range, which is attributed to the sulphonate ionising groups with a pKa value of two. Sulphur is mostly incorporated into the lignin molecule as sulphonate, while sulphate and sulphite could also be present which could render the 
lignosulphonates water-soluble. Other groups that can form polar groups include phenolic hydroxyl or carboxylic acid groups. The colloidal, surfactant and adhesive properties of lignosulphonates could be attributed to the high density of functional groups present (Ekielski \& Mishra, 2021). Lignosulfonates contain a higher degree of impurities compared to lignin from other pulping processes, which could complicate further processing for high-value applications (Macfarlane et al., 2014). Sulphonate groups incorporated onto the lignin structure during ether bond cleavage renders the lignosulphonate hydrophilic, which could be undesirable for resin applications. (Chen, 2014). Lignosulphonates also have high sugar contents from hemicellulose attached to the lignin and are insoluble in organic solvents (Lora, 2008). Unfortunately, removal of impurities present is not easily obtained (as discussed further in section 2.1.7), because the lignosulphonates could be very hydrophilic (Belgacem \& Gandini, 2008). The quantity of phenolic hydroxyls, aliphatic hydroxyls and carbonyl groups present, which determines the reactivity of the lignosulphonate, is greatly affected by the production/isolation process, molecular weight, and the purity of the lignosulphonates. There are various applications in which lignosulphonates could be utilised, due to their versatile characters. Currently, they are mostly utilised as dispersants, such as concrete plasticisers, drilling mud thinners, coal slurry and dye dispersants (Shao et al., 2017). Furthermore, they have the potential for use in soil conditioning, flotation, chelating, dust binding, and emulsion stabilising (Ruwoldt, 2020). Lignosulphonate may also be considered surfactants due to their large surface activity, however, they lack the one-dimensional structure with a hydrophilic-head and hydrophobic-tail structure as found in simpler surfactants.

\subsubsection{Chemical structure of lignin}

Lignin is a natural phenolic biopolymer made up of three main cinnamyl alcohols termed monolignols. It has an amorphous and highly cross-linked structure, where biosynthesis occurs by free radical generation, followed by coupling polymerisation of monolignols (Belgacem \& Gandini, 2008; Lu \& Ralph, 2010). During the biosynthesis of lignin, different bonds form, either between two monolignols forming dimers or between two polymer structures. 5-5 or 4-0-5 bonds could form between the two polymers (Lu \& Ralph, 2010), while $\beta-0$ $4, \beta-5$ or $\beta-\beta$ bonds could form between monolignols. Monolignols are named after their aromatic moieties: Coumaryl alcohol is named after a para-hydroxyphenyl (H-units) moiety. Coniferyl alcohol is named after its guaiacyl (G-units) moiety and sinapyl alcohol is named after syringyl (S-units) moiety. The basic structure of a monolignol is a phenolic compound with a propane side chain attached, with a methoxyl group that could also be attached to the ortho-positions, either on only the $C_{3}$ in the $\mathrm{G}$-unit or on both the $\mathrm{C}_{3}$ and $\mathrm{C}_{5}$ as in the S-unit, relative to the phenolic group, The most reactive sites are the aliphatic $\beta$-carbon and the phenolic hydrogen. The ortho-positions on the phenolic ring also have high free electron densities, however, their reactivity will decrease when these sites are occupied by methoxyl groups in the G- and S-units (Lu \& Ralph, 2010). G- and $H$-units could either form $\beta-0-4, \beta-5$ or $\beta$ - $\beta$ bonded dimers, while only $\beta-0-4$ and $\beta$ - $\beta$ bonded dimers could be formed from S-unit dimerization (Chen, 2014; Lu \& Ralph, 2010). S-unit radicals cannot couple onto $C_{5}$, as they are restricted to form either $\beta-0-4$ or $\beta$ - $\beta$ dimers. Figure 1 shows the structure of the monolignols and Table 2 shows the composition of each in different biomass sources. 
<smiles>Oc1ccccc1</smiles>

phenol

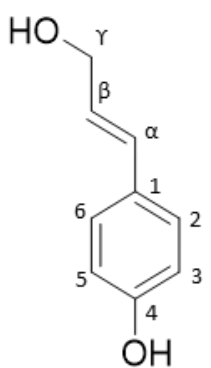

para-coumaryl alcohol

(H-unit)<smiles>COc1cc(/C=C/CO)ccc1O</smiles>

coniferyl alcohol

(G-unit)<smiles>COc1cc(/C=C/CO)cc(OC)c1O</smiles>

sinapyl alcohol

(S-unit)

Figure 1. Structures of the three monolignols. Redrawn from Pfungen (2015).

The methoxyl groups present on the units lowers the number of reactive sites that are available for the reaction with formaldehyde, which lowers the reactivity of the units. Therefore, the H-units will be the most reactive, followed by the G-units and then the S-units. Depending on the biomass source, the fractions of each unit present differ, as demonstrated in Table 2. Softwoods consist mostly of G-units, with a small number of H-units and only trace amounts of S-units. Hardwoods are mostly made up of S-units but can also be formed from a substantial quantity of G-units and a small quantity of H-units. Grasses show a more equal distribution of all three units, with a contribution decreasing in the order $\mathrm{G}>\mathrm{S}>\mathrm{H}$ units.

Table 2. The average contribution of the principal phenolic building blocks in lignin for different biomass sources. (Gellerstedt et al., 2009).

\begin{tabular}{lccc}
\hline Biomass source & H-units (\%) & G-units (\%) & S-units (\%) \\
\hline Softwoods & $<5$ & $>95$ & Trace amounts \\
Hardwoods & $<8$ & 25 to 50 & 46 to 78 \\
Grasses & 5 to 33 & 33 to 80 & 20 to 54 \\
\hline
\end{tabular}

Different bonds may be present in the lignin structure: The $\beta-0-4$ bond is the most dominant in softwood, hardwood and grasses and it is the bond most affected by treatment methods as it easily cleaved. In comparison, grasses have a higher content of $\beta-0-4$ bonds, followed by hardwoods, then softwoods. Although softwood contains a large amount of G-units, it is the most challenging to degrade in pulping as it is highly condensed and has a high degree of cross-linking and branching (Ekielski \& Mishra, 2021). The $\beta-\beta$ bonds in hardwood may degrade faster than that of the softwood lignins, leading to more reactive sites available (Gellerstedt et al., 2009; Henriksson et al., 2010). The amount of $\beta$-1 bonds for hardwood and softwood are approximately equal. The $\mathrm{H}$-units in grasses are mostly connected with $\beta-\mathrm{O}-4$ bonds (Chen, 2014), which are also then easily degradable. S-units in hardwood, which have two methoxyl groups, form weak ether linkages ( $\beta-0-4, \alpha-0-4,4-0-5)$, resulting in a lower molecular weight after pulping with not a lot of branching between the lignosulphonate units. In contrast, the $\mathrm{H}$ - and G-units which have either no or one methoxyl group, respectively, could form stable $\mathrm{C}$ - $\mathrm{C}$ bonds during either the biosynthesis of the plants or pulping in alkaline conditions, which are harder to break in the pulping process. Therefore, a more branched lignin network could 
be expected for lignins with more $\mathrm{H}$ - and G-units. It may be expected that lignin from biomass sources with a larger number of the more reactive units may be easier to utilise, however other factors (such as the linkages present or the degree of branching) also have a big effect on the utilisation. Even though hardwood lignins have less reactive units, they have proven to perform better than softwood lignins in LPF resins in some cases, due to their different bonds and more linear structure (Lourençon et al., 2020). Technical lignins valorised from the pulping industry could further undergo concentration of black liquor, precipitation of black liquor, purification, washing and drying stages. All the above mentioned influences the structure and properties of the lignin (Sixta, 2006; Lu \& Ralph, 2010). Technical lignin from the pulping industry thus has a relatively lower molecular weight, as native lignin structures are broken down during processing (Wang \& Venditti, 2015).

\subsubsection{Properties of lignin}

Lignin has many desirable properties - it is non-toxic, environmentally friendly, lightweight, low costs, and high in energy content (Ferdosian et al., 2017). It is more heat resistant than cellulose and hemicellulose (Amen-Chen, Pakdel \& Roy, 2001), it contains chromophore functional groups which can absorb UV radiation (Huang, Liu \& Qiu, 2019), and it may protect plants against biological attack by impeding enzyme penetration (Rowell, 2005; Wool \& Sun, 2005). Desirable properties of lignin for utilisation in LPF resins include high hydrophobicity, high purity, low glass transition temperature, low molecular weight, and low polydispersity. Table 3 shows the different properties of lignins obtained from different pulping processes.

Table 3. Properties of technical lignins (Lora, 2008; Laurichesse \& Avérous, 2014; Ruwoldt, 2020).

\begin{tabular}{lccc}
\hline & \multicolumn{2}{c}{ Sulphur-containing lignins } & Sulphur-free lignins \\
\hline Lignin type & Kraft & Lignosulphonate & Soda \\
Raw materials & Softwood/hardwood & Softwood/hardwood & Annual plants \\
Solubility & Alkali/organic solvents & Water & Alkali \\
$\mathrm{M}_{\mathrm{w}}(\mathrm{g} / \mathrm{mol})$ & 1500 to 25000 & 1500 to 50000 & 1000 to 15000 \\
$\mathrm{M}_{\mathrm{n}}(\mathrm{g} / \mathrm{mol})$ & 1000 to 3000 & 15000 to 50000 & 800 to 3000 \\
Polydispersity & 2.5 to 3.5 & 6 to 8 & 2.5 to 3.5 \\
$\mathrm{~T}_{\mathrm{g}}\left({ }^{\circ} \mathrm{C}\right)$ & 140 to 150 & 130 & 140 to 185 \\
$\mathrm{~T}_{\mathrm{d}}\left({ }^{\circ} \mathrm{C}\right)$ & 452 & 450 & $\mathrm{NR}^{1}$ \\
Ash content $(\%)$ & $<3.0$ & $\mathrm{NR}$ & $<1.0$ \\
Sugar content $(\%)$ & $<2.3$ & $\leq 35$ & 2.0 to 3.0 \\
Sulphur content $(\%)$ & 1.5 to 3.0 & 4 to 8 & Sulphur free \\
\hline
\end{tabular}

${ }^{1} \mathrm{NR}$ : not reported.

Native lignins are attached to cellulose and hemicellulose by strong inter-molecular bonds, making them insoluble in water or any solvent in their native state (Chen, 2014). Nonetheless, after the cooking process, lignin is separated from its fibre fraction containing polysaccharides and becomes soluble based on the method of isolation. The solubility of lignin in alkaline solutions is dependent on the ionising functional groups content, such as carboxyl, and sulphonate groups (Chen, 2014). Lignin obtained from the Kraft and soda processes is not water-soluble, whereas lignosulphonates from the sulphite pulping processes are water-soluble, as the incorporation of hydrophilic sulphonate groups on its amorphous structure occurs (Abdelaziz et al., 2016). 
The purity of lignin is affected by the amount of cellulose, hemicellulose, and other chemicals either from the pulping process, isolation methods or from the biomass source still present in the lignin. Ash only degrades at very high temperatures and different purification methods have proven to help remove the impurities present in the lignin. The ash content in native wood is usually less than one percent and is mainly constituted of calcium, potassium, and magnesium, with small amounts of sodium and silicon that could be present. Herbaceous plants could contain much higher silicon contents than wood species which could contribute to higher ash content. The temperature, time and $\mathrm{pH}$ used during the isolation process to separate lignin from black liquor could also affect the ash content (Kalami et al., 2018). The ash content in lignins is however mainly determined by the pulping method, with the pulping chemicals contributing much more to the ash content than the biomass source does (Sameni et al., 2014). Lignosulphonates which use sulphur dioxide in their pulping process could have a low purity, because of the sulphur that is used. Chemicals used for neutralisation or purification processes of lignins could also contribute to a lower purity (Kalami et al., 2018). A high ash content could hinder the reactivity of lignin. It could cause undesired side reactions, and it can restrict the penetration of the LPF resin on the plywood which reduces the bond line strength (Yang et al., 2015). Therefore, low carbohydrate and ash contents tend to produce better LPF resin performance (Wang \& Chen, 2014). However, a higher ash content could contribute to a higher thermal stability of the products formed, as the ash only degrades at very high temperatures (Alonso et al., 2011), but it could also cause a catalytic effect leading to lignin degradation at a lower temperature (Naron et al., 2017) and could also decrease the glass transition temperature $\left(T_{g}\right)$ of lignin (Sameni et al., 2014).

Native lignins are amorphous and thermoplastic. The behaviour of lignin is influenced by temperature, as lignin is known to be a thermoplastic. The $T_{g}$ is defined as the temperature at or above which a molecular structure transitions to a more flexible state (Crawford \& Throne, 2002). Above the $T_{g}$, lignin becomes soft, formable, and tenacious and exhibits adhesive forces. It then hardens again as it cools down. The $T_{g}$ of lignin could range from 114 to $150{ }^{\circ} \mathrm{C}$ (Huang et al., 2019) and it is directly proportional to the molecular mass thereof (Laurichesse \& Avérous, 2014). It varies depending on the isolation method, molecular weight, sorbed water and the thermal history of the lignin. At temperatures below $T_{g}$, the polymer is in a solid glass state and only internal and localised movements occur. At temperatures above $T_{g}$, the polymer undergoes radical-induced self-condensation (Wang \& Venditti, 2015). It is then less viscous and flows more easily, especially in the presence of water, as segmental motions of three to five units occur rendering the chain more mobile. Increasing hydration of lignin will also increase the dynamics thereof and water thus acts as a plasticiser to lignin (Laurichesse \& Avérous, 2014; Vural, Smith \& Petridis, 2018). The $T_{\mathrm{g}}$ could be determined by differential scanning calorimetry or other techniques such as dilatometry, and viscoelastic measurements.

The thermal decomposition temperature $\left(T_{d}\right)$ is the temperature at which lignin starts decomposing. The decomposition of lignin involves a multitude of reaction pathways, due to its complexity and the steric hindrance present in its structure. The decomposition temperature range is large due to various bonds, aromatic and aliphatic moieties present, which all decompose at different temperatures. Lignin decomposition is slower and could occur at a broader temperature range $\left(200\right.$ to $\left.500{ }^{\circ} \mathrm{C}\right)$ than cellulose and hemicellulose (Brebu \& Vasile, 2010). The highest rate of decomposition of lignin usually occurs between 360 to $400{ }^{\circ} \mathrm{C}$. Decomposition could start at temperatures between 150 and $275{ }^{\circ} \mathrm{C}$, where it is usually the dehydration of 
hydroxyl groups on the benzyl group that degrade first resulting from propyl chains that could have cleaved above $180{ }^{\circ} \mathrm{C}$ (Collard \& Blin, 2014). The $\alpha$ - and $\beta$-aryl-alkyl-ether linkages are cleaved next between 150 and $300{ }^{\circ} \mathrm{C}$. At $300{ }^{\circ} \mathrm{C}$ the aliphatic side chains cleave from the aromatic ring, and at 370 to $400{ }^{\circ} \mathrm{C}$ cleavage of the inter-unit $\mathrm{C}-\mathrm{C}$ bonds occur. The complete backbone is rearranged between 500 and $700^{\circ} \mathrm{C}$ which leads to $30 \%$ to $50 \%$ ash formation and the release of volatiles, such as $\mathrm{CO}, \mathrm{CO}_{2}, \mathrm{CH}_{4}$ and $\mathrm{H}_{2}$ (Laurichesse \& Avérous, 2014). A polydispersity index (PDI) is a measure of the width of the molecular weight distribution, which indicates the linearity of polymers. A low PDI will correlate with a more linear distribution (Rogošić, Mencer \& Gomzi, 1996). A PDI of one indicates that a molecule is monodispersed, with all its chains having the same length, which is for example the case in proteins (Shrivastava, 2018). The PDI could be calculated as the ratio of the mean weighted average molecular weight $\left(M_{w}\right)$ to the mean number average molecular weight $\left(M_{n}\right)$. The average molecular weight for lignin is affected by the biomass source and the isolation technique, resulting in a wide possible range (Glasser, Davé \& Frazier, 1993). During the pulping processes, lignins react and are depolymerised in different ways, leading to different molecular weights depending on the severity of the pulping process. The higher PDI of lignosulphonates indicate that they are less linear than Kraft and soda lignin, which could be attributed to sulphite pulping where multiple reactions could occur.

\subsubsection{Hydrophilic nature of lignosulphonates}

All lignins have one thing in common, they contain hydrophobic aromatic and aliphatic groups. The hydrophilicity of lignins is influenced by the presence of carboxyl and phenolic hydroxyl groups which form negatively charged anions in an alkaline medium (Ruwoldt, 2020). In lignosulphonate, the hydrophilicity is significantly enhanced depending on the count of sulphonate groups introduced. (Yan \& Yang, 2015). However, not all functional groups increase the hydrophilicity (ketones, aldehydes, and methoxyl groups), while ether and aliphatic hydroxyl groups could function as hydrophilic groups, depending on the molecular structure surrounding them (Ruwoldt, 2020). Hemicellulose is the wood constituent that is the most hydrophilic and partly water-soluble (Pelaez-Samaniego et al., 2013), and could therefore attribute to the hydrophilic nature of the lignosulphonate if carbohydrates are attached to the lignin structure. It has been suggested that hydrophobic moieties aggregate to the core, while hydrophilic moieties aggregate to the surface of lignosulphonates (Rezanowich, Yean \& Goring, 1964), as it has a hydrophobic high molecular weight aromatic structure, with hydrophilic moieties attached to the unit (Matsushita, 2015). Protonation of phenolic groups on lignin leads to less electrostatic repulsion between lignin molecules, which renders the molecules less hydrophilic (Hidayati, Satyajaya \& Fudholi, 2020), which is the case when alkaline lignin is precipitated by mineral acids out of black liquor. High hydrophilicity and anionic groups are undesirable in LPF resins as they could cause high moisture absorption and weak adhesion, while aromatic and aliphatic moieties will interact with hydrophobic surfaces and interfaces (Ruwoldt, 2020).

\subsubsection{Acid precipitation and purification of lignin}

When lignins are isolated from black liquor by acid precipitation, protonation of the phenolates occur, which reduces the electrostatic repulsion forces, reduces the hydrophilicity, and causes the lignin to flocculate. Lignin precipitation can start at a high $\mathrm{pH}$ and continues as the $\mathrm{pH}$ decreases. At a pH of two, most of the lignin should be precipitated (García et al., 2009). Acid precipitation and acid purification could alter the properties of the 
lignin, as it could lead to less branching in the lignin structure, which could result in cleavage of ether bonds at harsh reaction conditions (Hidayati et al., 2020; Zhang, Fu \& Chen, 2020). The solubility is affected by the molecular weight of the lignin and the ratio of the anionic groups to aromatic units(Ekielski \& Mishra, 2021).

\subsubsection{Membrane purification of lignosulphonates}

Most high-value applications desire minimal impurities to improve the reactivity of the lignin and to prevent side reactions from occurring. An ash content below $5 \%$ is generally desired (Fatehi \& Chen, 2016). For LPF resin applications, Kalami et al. (2018) proved that the adhesive shear strength was not sensitive to ash content. However, it should be noted that the highest ash content included was $11.4 \%$. Lignosulphonates have a higher ash content than other lignins, as discussed in section 2.1.4. During sulphite pulping, ether bonds are cleaved between the phenylpropanoid moieties and sulphonic acid groups are introduced onto the aliphatic chains of the lignin, which results in water-soluble and surface-active lignosulphonates formed (Angelini et al., 2019). Impurities in technical lignins could include sugars, silicates, sulphur, proteins (nitrogen in organic form) and other compounds which could either be from the biomass source, pulping method or extraction process (Vishtal \& Kraslawski, 2011). Impurities in the form of carbohydrates are common in technical lignins and are especially abundant in lignosulphonates from acidic sulphite pulping. Carbohydrates could be cross-linked to the lignin polymer and could occupy possible reactive sites, thereby reducing the reactivity of the lignin and processing possibilities. A high impurity content could cause a decrease in quality, deterioration of lignin properties, formation of undesirable by-products, and lower product yields (Khokarale, Le-that \& Mikkola, 2016). For example, sulphur present from sulphite pulping could poison catalysts used in chemical processing (Vishtal \& Kraslawski, 2011). It is therefore desirable to remove these impurities (for example by desulphurisation) before valorisation of lignosulphonate to improve the reactivity thereof (Renedo \& Fernández-Ferreras, 2016; Parto et al., 2019).

Most inorganic salts and other water-soluble impurities could be removed from the lignin by water washing. However, removing impurities in lignosulphonates are challenging as both the lignosulphonate and carbohydrates are soluble in water and are cross-linked to each other (Lora, 2008). Lignosulphonates are nontoxic; however, their dark brown colour causes undesirable high turbidity in water, and their high organic content is considered an environmental pollutant with dissolved organic carbon (DOC) acceptable in industrial waste waters. It is therefore desired to remove lignosulphonates from waste streams to produce water that could be reused. These lignosulphonates could then be utilised for high-value applications. Filtration and precipitation are two well-established methods of purifying lignosulphonates, while more are currently being investigated. Other possible water purification approaches include amination, carboxylation, cross-linking, depolymerisation, etc. Although membrane technologies have proven to be efficient in producing pure lignosulphonates (Ringena, Saake \& Lehnen, 2005), there are several drawbacks to them. They are uneconomical processes as the membranes are expensive, they have high operating costs, they require daily cleaning, and membranes need to be replaced every twelve to fifteen months. They are also susceptible to fouling and increasing accumulation of surface charge, which causes a decreased flux across the membranes (Xian et al., 2014; Aro \& Fatehi, 2017). 
Dialysis could be used to remove impurities such as hemicellulose, simple sugars, and inorganics (e.g., sulphur, sulphonic acids, ash, and salts) from lignosulphonate. In dialysis, the molecular weight cut-off (MWCO) refers to the smallest average molecular weight of a standard molecule that does not effectively diffuse across the membrane. Lignosulphonates have reported molecular weights of 1500 to $50000 \mathrm{~g} / \mathrm{mol}$ (Ruwoldt, 2020). Sulphonic groups in sodium lignosulphonate have been reported to exist in the molecular weight range of 2471 to $4297 \mathrm{~g} / \mathrm{mol}$ (Madad et al., 2011). Marques et al. (2009) purified black liquor from magnesium sulphite pulping with Eucalyptus Globulus hardwood by dialysis. Dialysis membranes with MWCOs of 2000 and 5000 Da were used to treat thick and thin black liquor against distilled water for eight hours. The sugar content has been reported to be mostly monosaccharides such as xylose $\left(M_{w}\right.$ of $\left.150 \mathrm{~g} / \mathrm{mol}\right)$ and xylooligosaccharides. Sulphonated oligomers $\left(\mathrm{SO}_{3} \mathrm{H}\right)$ with low molecular weights (1000 to $1300 \mathrm{Da}$ ) were mostly present in the lignosulphonates. Angelini et al. (2019) filtered calcium lignosulphonate from a paper mill using a $3500 \mathrm{Da}$ dialysis membrane to remove hemicelluloses, simple sugars, and inorganic contaminants. A $10 \mathrm{wt} \%$ aqueous calcium lignosulphonate solution was made and filtered, whereafter the filtrate was dialysed with water for twelve hours. The dialysis removed polysaccharides and contaminants from the lignin. Membrane treatment could however have certain challenges and it could not be a very efficient process: Some low molecular weight fractions of lignosulphonate could also be removed along with impurities (Marques et al., 2009); however, this is undesirable as these are the most reactive units for LPF resin synthesis. Furthermore, impurities that are attached to lignin moieties are not cleaved and removed during dialysis treatment, which hinders the removal of all of the impurities.

\subsection{Phenol formaldehyde resins}

PF resins are produced by the polycondensation of phenol with formaldehyde in alkaline media and they are currently the second most used resins (after urea formaldehyde) in wood applications (Eckelman \& Eckelman, 1997; Pizzi, 2017). PF has been abundantly used in wood applications, such as plywood and particleboard, due to its durability, water resistance and mechanical strength (Yaakob et al., 2021). Other applications could include metal casting, electrical insulation, automotive parts, etc (Xu et al., 2019). PF resins have many desirable properties: they are more moisture-resistant than wood, they could withstand elevated temperatures and chemical ageing, and they have high wet and dry bonding strengths (Eckelman \& Eckelman, 1997). Initially, high reaction temperatures and slow curing processes were required, but over the years the synthesis process improved resulting in higher mechanical performance and lower formaldehyde emissions. The main factors determining the type of resin produced include the raw material chemical structure, catalyst type, $\mathrm{pH}$ value, and the phenol to aldehyde ratio used.

Two types of phenolic resins can be produced: Either novolac resins in acidic conditions or resole resins in alkaline conditions. Novolac resins are thermoplastic, synthesised in an acidic medium with a F/P molar ratio of less than one (Fink, 2013; Xu et al., 2019), and they require an additional catalyst to cure (Sarika et al., 2020). Resoles are thermosetting phenolic resins synthesised in a basic medium and a F/P molar ratio of more than 1. Advantages of resoles include that they could form large three-dimensional networks with a high elastic modulus and tensile strength. They are cost-effective, moisture resistant, and have high thermal and mechanical stability. Resoles synthesised under basic conditions could also cross-link better without additives 
(Pizzi, 2006). Unfortunately, resoles produced could still contain a large amount of free formaldehyde, if it is in high excess in the synthesis reaction. Other disadvantages could include slow hardening compared to amino plastic resins, and a dark colour which could be undesirable in many applications (Pfungen, 2015).

The resole resin synthesis occurs following three polymerisation reaction sequences: addition, condensation, and curing. First is the addition of formaldehyde onto the phenol units, also known as a hydroxymethylation or methylolation reaction. Formaldehyde reacts with the active ortho- and para-sites on the phenolic ring (Tachon, Benjelloun-Mlayah \& Delmas, 2016). The reaction is carried out with excess formaldehyde around 60 ${ }^{\circ} \mathrm{C}$ in the presence of an alkali metal hydroxide (usually sodium hydroxide), giving a pH value between 8 and 13 (Tonge, 2007). Mono-methylolphenol, two di-methylolphenols and one tri-methylolphenol are formed, as shown in Figure 2. It is desired that as much as possible methylol groups attach to form large, cross-linked structures during condensation and curing. It is also desired that most of the formaldehyde reacts for economic benefit and environmental safety so that resoles with a low free formaldehyde content are formed. Keeping the reaction temperature around $60^{\circ} \mathrm{C}$ prevents condensation of methylol functional groups on the units, giving the formaldehyde a chance to react completely before cross-linking occurs which causes steric hindrance and hinders the further addition of formaldehyde.

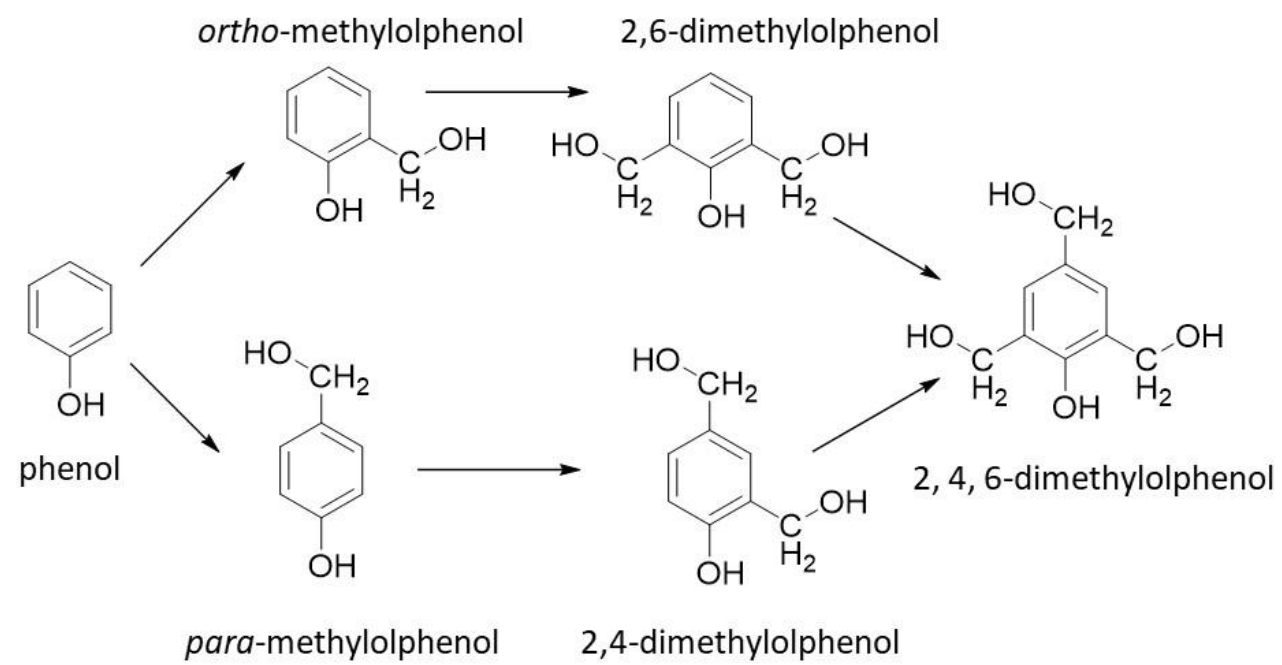

Figure 2. Reaction paths for the addition of formaldehyde to phenol. Redrawn from Tonge (2007).

Secondly, condensation of the methylol phenols occurs upon heating from 60 to $100^{\circ} \mathrm{C}$ and forms methylene or methylene ether bridges. Two methylol groups could react to form a dimethylene ether bridge with the release of a water molecule. Methylene bridges are formed either when two methylol groups react and simultaneously releases one molecule of water and one molecule of formaldehyde, or else when a methylo reacts with a proton on the ortho- or para-sites of the aromatic ring, releasing only a molecule of water (Tonge, 2007). As the condensation and hence cross-linking occurs, the molecular weight increases, which leads to an increase in viscosity. When the desired viscosity is reached, the reaction mixture is cooled to around 25 to 40 ${ }^{\circ} \mathrm{C}$ to suppress further reactions from occurring (Pizzi, 2017).

Finally, the curing reactions of the resole take place during hot-pressing, upon application of the resin, performed at temperatures between 125 and $150{ }^{\circ} \mathrm{C}$ without requiring a curing agent (Siddiqui et al., 2017). 
It is a thermally activated process, by which the low molecular weight reactants are transformed to highly cross-linked networks. Heat is applied and the methylol groups cross-link further until a highly branched and cross-linked polymer is formed (Tonge, 2007). Resoles could remain stable for three to nine months and typical properties include a viscosity between 0.1 and $0.2 \mathrm{~Pa}$.s at $20^{\circ} \mathrm{C}$, a $55 \%$ to $60 \%$ solids content, and a pH value between 7 and 13 (Pizzi, 2017).

In PF batch manufacturing a F/P ratio of 1.1/1 and catalyst ammonia fraction of $1 / 40$ of the total mass of the batch is used (Malutan, Nicu \& Popa, 2008). PF resole synthesis reactions are exothermic and runaway reactions could occur if the heat produced during the chemical reaction exceeds the cooling capacity of the vessel. Once the reaction is initiated, the heat generated favours the forward reaction, which increases the reaction rate and produces more heat (Bhattacharjee, Neogi \& Das, 2014). This could lead to an increase in temperature, and in some cases increased pressure as well if not controlled. PF resin synthesis is typically performed in an insulated stainless steel batch reactor containing an agitator and a reflux condenser. A formalin solution ( $35 \%$ to $42 \%$ aqueous formaldehyde) is added to molten phenol in an alkaline catalyst, typically sodium hydroxide, which keeps the reagents in solution. Other possible catalysts could include alkaline oxides, hydroxides, ammonia, or sodium carbonate, which all form alkaline solutions and provide HOgroups.

\subsection{Lignin phenol formaldehyde resins}

Phenol is derived from the petroleum industry; it is toxic, non-renewable, and there is a lot of fluctuation in the price and availability thereof. Therefore, it would be beneficial to find a more sustainable alternative to partially substitute or replace phenol used in industry. Biopolymers have proven to be more environmentally sustainable than synthetic polymers (Dessbesell et al., 2020), therefore lignin utilised as a bio-based adhesive has drawn lots of interest due to its phenolic structure and the abundance of technical lignins available (Hu et al., 2011). It has proven to be an alternative to petroleum for energy, chemicals and materials (Dessbesell et al., 2020). Lignin could be a more environmentally sustainable replacement for phenol in producing LPF resins because it is a green renewable feedstock, it is non-toxic, it has lower energy requirements (Tonge, 2007), and it also has a longer storage stability than phenol (Solt, van Herwijnen \& Konnerth, 2019). However, it has a reduced reactivity compared to phenol which leads to longer pressing times and higher pressing temperatures (Matsushita, 2015). Some of the main barriers and incentives of substituting phenol with lignin in LPF resins have been identified as economic viability, process operating conditions, process reliability, stable supply availability, environmental benefit and collaboration along the value chain (Lettner et al., 2020).

\subsubsection{LPF synthesis and curing process}

The synthesis of LPF resins is like that of PF synthesis. The synthesis of LPF resins is like that of PF synthesis. A certain weight percentage of the phenol can be substituted by lignin, which is then expressed as the percentage substitution. The phenol, lignin and formaldehyde are then reacted in alkaline conditions for the LPF synthesis to occur. During the synthesis process, three reactions could occur; namely, the LedererManasse, Cannizzaro and Tollens reactions (Alonso et al., 2001). The Cannizzaro reaction is undesirable, as formaldehyde condenses with itself leading to a decreased viscosity (Alonso et al., 2004; Malutan et al., 2008). During the Tollens reaction, formaldehyde attaches onto side-chain carbonyl groups, introducing reactive 
methylol groups (Matsushita, 2015), which aids in forming cross-linked networks. However, the LedererManasse reaction is the most desirable reaction for LPF synthesis: During the addition reaction, the formaldehyde could be linked via electrophilic substitution under alkaline conditions onto the free orthopositions of the G- or H-units (Matsushita, 2015), thereby incorporating methylol groups onto the lignin ring as shown in Figure 3. Marton, Marton, Falkehag \& Adler (1966) found that the number of methylol groups introduced by the Lederer-Manasse and Tollens reaction is in a ratio of three to one for the Kraft pine lignin they investigated. Zhao et al. (1994) reported that during methylolation of a pine Kraft lignin 0.36 mol hydroxymethyl groups per $\mathrm{C} 9$ unit were introduced, of which 0.33 mol attached to the $C_{5}$ of the G-unit via the Lederer-Manasse reaction.

The S-units of lignin have both ortho-positions occupied by methoxyl groups, leading to low reactivity, which hinders the addition of formaldehyde onto the aromatic ring. G-units have one reactive site available on the ortho-position, while the other is occupied by a methoxyl group. The H-units have both ortho-positions open as available reactive sites (Ferdosian et al., 2017). Therefore, -units with the highest reactivity would be most favourable for LPF synthesis is thus the highest, followed by G- and then S-units, as shown in Figure 4. Furthermore, some free phenolic hydroxyl groups could be involved in ether linkages formed between lignin units and are thus not available to form phenolate ions which act as electron donors in the aromatic substitution (Ghorbani et al., 2016).<smiles></smiles>

Formaldehyde $+\mathrm{G}$ monomer $\rightarrow$ Methylolated lignin derivative

Figure 3. Methylolation reaction between formaldehyde and a G-unit of lignin. Reproduced from (Hemmilä et al., 2017).<smiles>Oc1ccccc1</smiles>

phenol

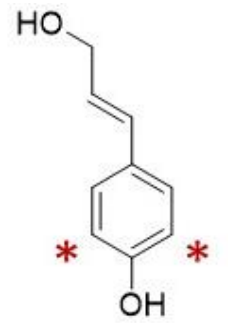

para-coumaryl alcohol

( $\mathrm{H}$ monomer)<smiles>COc1cc(/C=C/CO)ccc1O</smiles>

coniferyl alcohol

(G monomer)<smiles>COc1cc(/C=C/CO)cc(OC)c1O</smiles>

sinapyl alcohol

(S monomer)

Figure 4. Representation of the reactive sites on phenol and the lignin units. Reproduced from Hemmilä, et al. (2017). 
The LPF synthesis could be performed on a laboratory scale in a three-neck round-bottom flask along with a condenser, thermometer, agitator, heating mantle, and cooling system. The phenol and lignin are reacted in an alkaline media (usually $\mathrm{NaOH}$ ) at a temperature between 45 to $65^{\circ} \mathrm{C}$. The formaldehyde (37\%) is slowly added, whereafter the temperature is increased to $65^{\circ} \mathrm{C}$. A sudden addition of large amounts of formaldehyde in the condensation reaction could cause a spike in the temperature, therefore a target temperature below $70^{\circ} \mathrm{C}$ is desired during the addition reaction as it is an exothermic reaction. Controlled constant formaldehyde dosing is ensured using a titration device or automatic burette to ensure minimum temperature fluctuations, constant viscosity development, and to avoid runaway reactions (Ghorbani et al., 2016). The condensation reaction is then initiated by adjusting the temperature to 80 to $85^{\circ} \mathrm{C}$ for one to two hours until the desired viscosity is reached. The condensation reaction temperature is usually targeted at this temperature, as too high temperatures could cause runaway reactions (Khan, Ashraf \& Malhotra, 2004; Ghorbani et al., 2016; Kalami et al., 2017).

During the condensation reaction, the methylol groups react to form either dimethyl ether or methylene bonds in the same manner as with PF synthesis (Siddiqui et al., 2017). A linear polymer is initially formed, which later cross-links to form a highly branched structure during the curing process (Hemmilä et al., 2017). The formed resin is then cooled to room temperature to stop further reactions, and then stored below $0{ }^{\circ} \mathrm{C}$ (Ghorbani et al., 2016). The sodium hydroxide catalyst could alternatively also be added incrementally throughout the condensation reaction, which could hinder the runaway reaction occurring (Yang et al., 2015). The final curing of the resin occurs upon application during the hot-pressing process, where the heat applied initiates the final condensation and cross-linking reactions to form a highly branched, three-dimensional polymeric structure.

Undesirable side reactions could occur during LPF synthesis at certain reaction conditions, such as at a too high temperature, $\mathrm{NaOH}$ concentration, $\mathrm{pH}$ value, or $\mathrm{P} / \mathrm{F}$ ratio. Undesirable reactions include the Cannizzaro reaction where formaldehyde condenses with itself leading to a decreased viscosity (Alonso et al., 2004; Malutan et al., 2008), or the Tollens reaction, where formaldehyde attaches onto side-chain carbonyl groups, introducing methylol groups (Matsushita, 2015). These side reactions decrease the amount of formaldehyde available that could attach as methylol groups on the aromatic ring, resulting in less cross-linking. This could lead to reduced bonding strengths and increased gelation times (Sarkar \& Adhikari, 2000).

\subsubsection{Effect of LPF synthesis and curing parameters on the resin performance}

There are several variables in the LPF resin synthesis that are dependent on each other. Different optimisation parameters that were used in previous reports are listed in Table 22 in Addendum A. A wide range of parameters is applied due to the varying properties of different lignins. In the reaction of phenolic compounds with formaldehyde to produce resole resins, the formaldehyde is in excess. A larger formaldehyde content will thus favour the forward reaction, which will lead to the addition of more formaldehyde and ultimately more cross-linking between methylol groups. An increase in F/P also leads to faster curing reaction rates yielding lower gel times, higher molecular weights, and higher viscosities (Sarkar \& Adhikari, 2000; Tonge, 2007; Siddiqui et al., 2017). The amount of formaldehyde used is very important, as the free formaldehyde produced is significantly influenced by the amount and reactivity of lignin used. At low reactivity of lignin, less 
formaldehyde will attach leading to a high free formaldehyde content in the product (Tachon et al., 2016). Therefore, if there is too much formaldehyde reactant, the product will contain free formaldehyde, which could decrease the strength of the resins and lead to high toxicity causing environmental concern (Tachon et al., 2016).

The reactivity of the different lignin building units vary (as discussed in section 2.1.3) and is lower than that of phenol, as it has side chains attached. Native lignin building units are linked to each other in a branched network. These e linkages between the units are reduced after pulping. At higher substitution levels of phenol by lignin, the higher branching of lignin would attribute to a higher molecular weight. Larger molecular weights aids in faster viscosity development of the LPF resins (Alonso et al., 2004). However, too large molecular weights could hinder the penetration of the LPF resin into plywood, hindering effective bonding (Yang et al., 2015).

The $\mathrm{pH}$ of the synthesis reaction also affects the LPF resin performance. The $\mathrm{pH}$ determines the degree of condensation and cross-linking formation of ether and methylene bonds. At a lower $\mathrm{pH}$ the condensation between phenol and formaldehyde could be incomplete, leading to a higher concentration of low molecular weight species, causing longer gelation times, and an increased free formaldehyde content (Sarkar \& Adhikari, 2000). A pH value between 9 and 11 is generally maintained for resole resins to avoid lignin precipitation and to maintain high water resistance. An increase in $\mathrm{pH}$ (between 9 and 11) leads to an increase in viscosity and shorter gelation times (Kalami et al., 2018). However, a too high increase in pH could cause unwanted side reactions to occur, such as Tollens or Cannizzaro reactions (Hemmilä et al., 2017). A too high increase in pH could therefore also cause a decrease in cross-linking, gel time and adhesive strength due to the formaldehyde consumed by the side reactions (Siddiqui et al., 2017).

The water-resistance of resins is an important factor when resins are used in applications that might be exposed to water. If sufficient cross-linking of the resin has not taken place during the curing process, the resin could react with the water and some of the resin could be lost, resulting in reduced bonding strength Kalami et al. (2018). More reactive lignin could form a LPF resin with better water resistance as it will lead to a higher degree of cross-linking that resists penetration and breakdown by water (Kalami et al., 2018). Solt et al. (2019) proved the distinctive thermoplastic behaviour of LPF resins and that they are not affected by moisture, as they will restore when returned to standard conditions, while PF resins have less moisture resistance when they are not fully cured.

The thermal stability of LPF resins has been reported to be higher than that of PF resins and they also exhibit less weight loss when stored or exposed to water. This was attributed to the higher number of strong bonds formed, which give high thermal stability (Sarkar \& Adhikari, 2000). During the synthesis process, the time and temperature need to be low enough if it will be implemented in industrial applications, but also sufficient for cross-linking reactions to complete. The synthesis of LPF resins is an exothermic reaction, so an increase in temperature increases the reaction rate and hence the curing rate (Bhattacharjee et al., 2014). The curing temperature of LPF resins is influenced by the degree of cross-linking of the LPF resin after condensation. High $\mathrm{F} / \mathrm{P}$ ratios lead to a higher degree of cross-linking and increased network formation; therefore, it could lead to decreased curing temperatures. At high substitution rates, the lignin dominates, leading to higher molecular 
weights and greater contact between bulk molecules which enhances the rate of curing and allows lower curing temperatures (Siddiqui et al., 2017). High lignin contents require a larger activation energy, due to lignin having low reactivity levels compared to phenol. It was found that lower curing temperatures and low activation energies led to faster completion of the synthesis reactions (Yang et al., 2015).

The curing process also has a lot of parameters that could be optimised during hot-pressing. A too high temperature could also cause degradation of the wood and the adhesive, and cause a decrease in the bonding strength ( $\mathrm{Gu}$, Huang \& $\mathrm{Li}, 2013)$. The hot-pressing time required is governed by the time it takes to reach a sufficient temperature in the inner glue to cure the resin. Too short hot-pressing times lead to incomplete reactions, while a too long time could cause decomposition of the adhesive and cause decreased bonding performance (Jing et al., 2015). Also, long-pressing times could render the process unproductive, and it could also reduce moisture content and produce panels that easily deform, which is undesired for industrial implementations.

Suitable hot-pressing conditions for plywood facilitates the penetration of the adhesive further into the wood panels, which could then form a nail structure and improve the strength of the plywood. However, excessive hot-pressing pressures could lead to over-compression of the veneers and could also cause too much adhesive to penetrate the wood, leaving insufficient adhesive to fill the bonding line. In contrast, too low pressures lead to insufficient contact and the formation of a poor adhesive layer resulting in low bonding strength (Bekhta, Sedliacik \& Bekhta, 2020). Generally, applying more adhesive increases the bonding area between the adhesive and the wood and this could increase the bonding strength as sufficient penetration increases the contact surface for bonding to occur (Ferdosian et al., 2017). However, an excessive application could again cause a too thick layer, due to the high viscosity, which then reduces the strength (Bekhta et al., 2020) and could lead to increased cost and defects on the bond line (Ferdosian et al., 2017). A more uniform adhesive distribution will lead to a stronger adhesive bond (Wang et al., 2012). The bonding strength and mechanical properties of wood adhesives could be evaluated by their shear strength, pull-off strength, or bending strength. In the adhesive shear strength test, the one end of each wood chip is glued by an adhesive, while the other end is inserted in a clamp on a tensile machine. The clamped is moved up at a certain speed until the bonded wood chips detach registering a force from which the shear strength could then be calculated (Ferdosian et al., 2017).

For particleboards, the density of the wood particles impacts the panel compaction ratio (which is the ratio of the panel density to the wood density), which must be high enough to ensure sufficient contact between the particles for enough densification of the panel. For boards produced with the same density, particles of lower density will lead to higher mechanical properties but could lead to reduced water absorption, thickness swelling and volume swelling (Iwakiri et al., 2019). It is beneficial if adhesives are used with natural fibres that could reduce the hydrophilicity of the fibre material containing hydroxyl groups (Dhakal, Zhang \& Richardson, 2007). A higher thickness swelling and water absorption could be caused by an increase in particle density, resin content and pressing time. The mechanical properties have been reported to increase with an increase in particleboard density, resin content, and pressing time (Iwakiri et al., 2019). 


\subsubsection{LPF resins at different substitution levels}

Several studies have been able to produce LPF resins with acceptable bonding strengths using different types of unmodified lignin. However, most studies have only been able to substitute phenol at low substitution levels to achieve acceptable bonding performance (Sarkar \& Adhikari, 2000; Donmez Cavdar, Kalaycioglu \& Hiziroglu, 2008; Park, Doherty \& Halley, 2008; Kouisni et al., 2011; Zhang, 2013; Martínez \& Velásquez, 2013; Moubarik et al., 2013; Yang et al., 2015; Siddiqui et al., 2017; Ghorbani, Mahendran, et al., 2018; Chen, Gong, et al., 2019; Solt, Jääskeläinen, et al., 2019). Kalami et al. $(2017,2018)$ proved that phenol could be replaced by a variety of unmodified lignin from the pulping industry to produce LF resins with acceptable properties for plywood applications. The LF resin proved to have a similar shear strength to a commercial phenol resorcinol formaldehyde resin when cured under the same conditions. However, wheat flour was used as an additive and alder bark (modal) was used as a filler during the adhesive formulation which aided in the increased shear strength compared to previous reports that did not use additives. Govender et al. (2020) used lignins from different sources from the paper and pulp industry of South Africa to synthesise LPF resins at $100 \%$ substitution level, aiming to achieve acceptable bonding strengths. None of the LPF resins produced was able to reach the shear strength of $0.70 \mathrm{MPa}$ (as specified by the Chinese Grade A plywood standard GB/T 14732-2013) without modification of the resin. The highest shear strength achieved was around $0.5 \mathrm{MPa}$. With resin modification by the addition of cross-linkers and hardeners (hexamine with either epichlorohydrin or glyoxal), some of the LPF resins were able to achieve the $0.70 \mathrm{MPa}$ benchmark. Yaakob et al., (2021) used lignin produced from empty fruit bunch using sulfuric acid and glacial acid, to synthesise a LF resin and achieved a shear strength of 1.76 $\mathrm{MPa}$. The addition reaction continued for two hours at $65^{\circ} \mathrm{C}$ and the condensation for four hours at 85 ${ }^{\circ} \mathrm{C}$. It was not reported if and how the resin was prepared as an adhesive for plywood and if additives were added during the adhesive formulation. Thus, no LPF resin at $100 \%$ substitution has been made utilising lignin from the pulping industry without modification or the addition of cross-linker or hardeners that succeeded in adhering to standard specifications for wood applications.

\subsection{Chemical modification methods to enhance lignin reactivity}

Due to the low reactivity and the complexity of lignin, a lot of modification methods have been investigated, aiming to increase the processability, chemical reactivity, and solubility of lignin in organic solvents. For a functionalisation method to be industrially viable, it should firstly be economically sustainable (having low capital and operating costs), to be able to compete with current commercial chemicals, such as phenol. Secondly, it should be environmentally sustainable and not contain more hazardous chemicals than commercially used. Ideally, it would also be advantageous if the functionalisation method could be applied to lignin from any biomass source, pulping or isolation process.

The use of unmodified lignin in LPF resin synthesis requires long pressing times and high pressing temperatures, because of the complex structure of lignin. The ortho-sites of the lignin building units could be occupied by methoxyl groups which cause steric hindrance and hence a lower reactivity compared to phenol (Hu et al., 2011). If phenol is substituted by lignin in LPF resin synthesis, this comparatively lower reactivity of lignin decreases the possibility of lignin reacting with formaldehyde. Different methods (chemical, mechanical, or biological) have been reported to improve the reactivity of lignin through either modification or 
depolymerisation (Matsushita, 2015). Chemical modification methods have been reported to have relatively low energy consumption compared to other methods and are the most promising currently to modify lignin for LPF resin applications (Matsushita, 2015).

The main chemical methods reported previously to increase the reactivity of lignin to produce LPF resins include demethylation, hydroxymethylation (also called methylolation) and phenolation (Hu et al., 2011). The reactivity of lignin is affected by the type of substitutions it has on its aromatic ring, where the most abundant functional groups on the lignin molecule are phenolic hydroxyl, aliphatic hydroxyl, methoxyl and aldehyde groups. However, most of these functional groups could be part of bonds formed between units in the lignin. Phenolic hydroxyls particularly are occupied in interunit linkages such as carbon-oxygen bonds ( $\beta-0-4, \alpha-0-4$, 4-0-5). The $\beta-0-4$ ether bond could represent $35 \%$ to $70 \%$ of the bonds present in the biopolymer structure, therefore cleavage thereof will release occupied functional groups. Therefore, the cleavage of this bond could be an effective way to improve the reactivity of the lignin by creating more sites for reactive hydroxyl groups (Klapiszewski et al., 2017). Phenolic hydroxyls are the most reactive functional groups present to take part in the polymerisation reaction during LPF synthesis with formaldehyde and it could significantly increase the reactivity of the lignin; therefore, a high concentration of phenolic hydroxyls could be favourable (Zhang et al., 2013; Laurichesse \& Avérous, 2014). However, it has also been proven that a too high phenolic hydroxyl content could promote non-covalent interactions between lignin moieties which then leads to the formation of a sterically hindered network formed, and it is proposed by some that the performance is rather governed by total hydroxyl content (Zhang et al., 2013).

\subsubsection{Hydroxymethylation}

During hydroxymethylation/methylolation, hydroxymethyl/methylol groups $\left(-\mathrm{CH}_{2}-\mathrm{OH}\right)$ are added to lignin molecules, which introduces more reactive sites onto the lignin molecule. The hydroxymethylation reaction occurs in similar alkaline conditions to the LPF synthesis where the lignin is first reacted with formaldehyde. Phenol is added later for the LPF synthesis to occur. Modifying lignin with a hydroxymethylation reaction for LPF resins containing phenol could be favourable as the formaldehyde addition on lignin is completed before phenol is added, while if phenol is present, the formaldehyde would first attach to the phenol as it has less steric hindrance. After formaldehyde addition to lignin is completed, the phenol is added and the formaldehyde could then further attach to the reactive sites on the phenol, whereafter condensation between methylol groups and reactive sites occurs further.

Advantages of hydroxymethylation include that it provides primary hydroxyl groups and electrophilic carbon atoms. The reaction is selective, and it could produce lignins with high reactive levels as the addition of methylol groups is favourable for condensation and cross-linking to occur during the resin synthesis reactions. Disadvantages include that only a low content of methylol groups could be generated and the bonding performance of materials obtained is moderate (Taverna et al., 2019). Moreover, it is a slow process and it fails in saturating all the reactive sites available (Stephanou \& Pizzi, 1993). It has however been implemented on an industrial scale at up to $30 \%$ substitution in North American plywood mills (Pizzi, 2006). Several studies have proved that hydroxymethylation of lignin is an effective means to improve the reactivity of lignin for 
application in LPF resoles at low substitution levels (Kaur, Uppal \& Sharma, 2017; Ghorbani, Konnerth, et al., 2018; Taverna et al., 2019).

\subsubsection{Demethylation}

Demethylation is also a promising method to improve the reactivity of lignin, as it removes either one or both methoxyl groups that could be present on the ortho-positions of the aromatic rings of the G- and S- units, reducing steric hindrance. They are replaced with highly reactive phenolic hydroxyl groups, with free electrons on the oxygen that stabilize the intermediate cation causing a high reactivity toward electrophilic aromatic substitutions on the ortho- or para-position (Hu et al., 2011). During the demethylation reaction, ether bonds of the methoxyl groups can be cleaved. Other ether bonds between lignin units could also be cleaved, which then forms more phenolic and aliphatic hydroxyls, and reduces the steric hindrance of the lignin units (Wang et al., 2019).

Several different methods with different catalysts have been tested to perform demethylation to improve the reactivity of lignin for use in LPF resins (Li et al., 2016; Song et al., 2016; Sawamura et al., 2017; Wang et al., 2019). Depending on the catalyst used, several disadvantages could occur, such as hazardous chemicals which could have a negative environmental effect, harsh operating conditions which are not desired in industrial implementation, costly raw materials, and high-pressure reactors are required that demands a large capital investment (Podschun et al., 2016). Some demethylation catalysts (such as sodium hydrosulphide) could also produce a pungent odour, which is undesirable (Li et al., 2017).

Biological demethylation of lignin by fungi and bacteria has been reported, but a high degree of depolymerisation of lignin could occur, which produces small molecular weight fragments (Wang, Zhao \& Li, 2018; Venkatesagowda \& Dekker, 2019). This could be unfavourable in LPF resin applications, as it could lead to a higher curing temperature and longer curing times. Demethylation could be carried out using solutions such as dimethylformamide along with surfactants that could be used, which could require post-treatment purification or separation before it is utilised leading to increased operational costs (Gao et al., 2021). Under severe demethylation conditions and aromatic ether cleavage, the demethylated material could be too reactive to survive the reaction conditions, thus either substantial decomposition occurs, or the polymer is converted to a different material. Sulphur-mediated demethylation has been implemented on an industrial scale in other processes and has therefore been reported to be a successful means for demethylation (Hu et al., 2011). Possible reagents could include S, NaSH, Na2SO3, and n-dodecyl mercaptan [3]. Sulphur dioxidemediated (Wu \& Zhan, 2001), and iodocyclohexane (Song et al., 2016) demethylation has proven to decrease the methoxyl group content and increase the phenolic hydroxyl content. However, these processes require high pressures, high temperatures, and long pressing times, which are not ideal for industrial implementation. $\mathrm{HBr}$ and $\mathrm{HI}$ have also been used to demethylate alkali lignin in another study and found that $\mathrm{HI}$ performed better, as it was a stronger nucleophilic reagent (Wang et al., 2019). Li et al. (2016) used four different demethylation catalysts ( $\mathrm{S}, \mathrm{NaSH}, \mathrm{Na}_{2} \mathrm{SO}_{4}, n$-dodecyl mercaptan) to demethylate agricultural crop-based soda lignin under atmospheric pressure and low temperature $\left(90^{\circ} \mathrm{C}\right.$ for 1 hour with a catalyst to lignin weight ratio of $10 \%$ ). All catalysts were able to demethylate lignin and were applied in producing $30 \%$ substituted LPF resins; however, a higher demethylation extent is still desired. Sodium hydrosulphide released a pungent 
odour, which is unsuitable for industrial implementation. Li et al. (2017) tried to improve the demethylation efficiency of alkali lignin by a nucleophilic substitution reaction using sodium sulphite as a catalyst under mild conditions. At optimal demethylation conditions a satisfactory demethylation efficiency was produced. The methoxyl content was found to decrease by $43.5 \%$, phenolic hydroxyls increased by $46.4 \%$ and aliphatic hydroxyls increased by $178 \%$. LPF resins were synthesised at $50 \%$ substitution with a bond strength increased from 0.92 to $1.07 \mathrm{MPa}$ and the formaldehyde emissions decreased from $0.58 \mathrm{mg} / \mathrm{L}$ to $0.22 \mathrm{mg} / \mathrm{L}$. Therefore, demethylation has proven to increase the reactivity of lignin at low substitution levels.

\subsubsection{Phenolation}

Phenolation is known to improve the reactivity of lignin, as phenol with new ortho- and para-reactive sites are introduced onto the lignin molecule, and cleavage of some weak bonds occur (Zhao et al., 2016). Condensation between phenol and lignin occurs, as phenol attaches to the available aromatic or aliphatic sites of the lignin units, thereby increasing the phenolic hydroxyl groups and the number of reactive sites on the lignin molecules (Jiang et al., 2018), as shown in Figure 5 . The phenol mostly attaches to the aliphatic $\mathrm{C}_{\alpha}$ position but has also been reported to attach to the $C_{\gamma}$ position (Podschun, Saake \& Lehnen, 2015). The $\beta-O-4$ ether bonds are the easiest to cleave; however, the side chain $\mathrm{C}-\mathrm{C}$ bonds in the $\beta-\beta$ substructure have also been reported to cleave (Lin, Nakagame, et al., 2001; Lin, Yao \& Shiraishi, 2001; Gan \& Pan, 2019). Cleavage of bonds between lignin units decreases the degree of cross-linking, which then forms more mono-dispersed fractions leading to a structure closer to that of phenol (Podschun et al., 2016; Kalami et al., 2017; Eraghi Kazzaz, Hosseinpour Feizi \& Fatehi, 2019). This reduction in higher molecular weight fractions could also improve the solubility and reactivity of the phenolated lignin with formaldehyde, as compared to unmodified lignin. Sulphonate groups could also be effectively cleaved during the phenolation of lignosulphonates (Hu et al., 2012). Phenolation could thus enhance covalent bond formation with formaldehyde and lead to less residual methoxyl groups, thereby reducing the steric hindrance and enhancing the reactivity (Thébault et al., 2020).

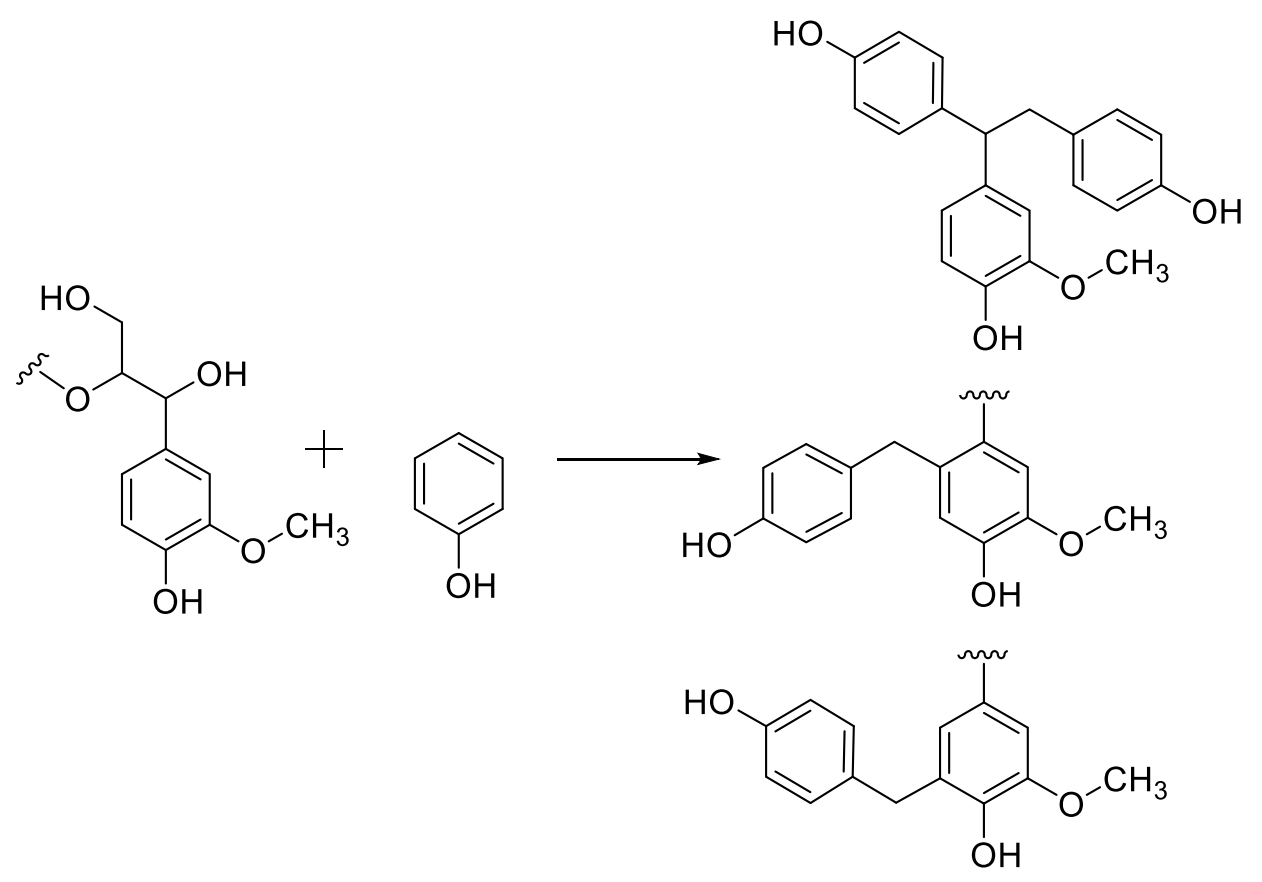

Figure 5. Addition of phenol onto the G-unit of lignin. Reproduced from Jiang et al. (2018). 
The depolymerisation of lignin that occurs during phenolation could also affect the $\mathrm{pH}$ of the medium, depending on if alkaline or acidic groups are released (Jiang et al., 2018). Phenolation mostly has an alkalising effect as the majority of the lignin fragments released are alkaline. However, some acidic fragments could also be released (Ragnar, Lindgren \& Nilvebrant, 2000). During this depolymerisation, a complex oligomeric mixture could be released which could have different effects when used in LPF synthesis. Depolymerisation of the lignin during phenolation could also lead to a lower viscosity. This will allow higher levels of substitution of phenol by lignin without causing a too high viscosity increase due to the high molecular weight of the lignin fraction incorporated (Thébault et al., 2020). Disadvantages of phenolation include that a large amount of formaldehyde is required as the extent of reaction is low due to the more branched network of the phenolated lignin. The reaction is also difficult to control and the number of phenols grafted to the lignin could be difficult to quantify (Podschun et al., 2016). A large amount of solvent is also required, which could reduce economic and environmental sustainability (Eraghi Kazzaz et al., 2019).

Phenolation has been performed with acid catalysts (Lee et al., in press; Ma et al., 2011; Podschun et al., 2016; Jiang et al., 2018; Thébault et al., 2020; Zhou et al., 2020) as well as alkaline catalysts (Yang et al., 2014; Zhao et al., 2016; Ghorbani, Konnerth, et al., 2018; Gan \& Pan, 2019). Phenolation reaction conditions using large amounts of acid could lead to a lot of acids produced that need to be neutralised, which is undesired environmentally and economically (Jiang et al., 2018). It has also been proposed that alkaline ash from the pulping process still present in alkaline lignin could inhibit an acid catalyst during phenolation (Podschun, Stücker, et al., 2015). Alkaline catalysts are more beneficial for phenolation before LPF synthesis as the synthesis is catalysed by alkalis, therefore it could then be incorporated into a one-pot process. A one-pot phenolation and LPF resole synthesis has been used where steam explosion lignin was phenolated in alkaline conditions, then transitioned directly into the resole synthesis reaction (Zhao et al., 2016). Gradual polymerisation occurred where phenol was added onto the lignin structure, whereafter the formaldehyde attached to available ortho- and para-positions on the phenolated lignin and finally, methylol groups condensed to form methylene or ether bridges. Alkaline catalysts thus promote less chemical waste, faster reactions, and easier transition into the synthesis reaction.

\subsection{Gaps in literature}

A lot of research has been conducted investigating the viability of lignin as a phenol replacement in LPF synthesis. Because of the diversity in different lignin properties, varying degrees of success have been reported. Several studies proved to substitute phenol by lignin at low substitution levels (Hu et al., 2014; Ghorbani, Konnerth, et al., 2018; Gan \& Pan, 2019; Yang et al., 2019). Only three studies have been able to replace phenol with lignin at 100\% substitution (Kalami et al., 2017, 2018; Govender, Majeke, et al., 2020; Yaakob et al., 2021). Govender et al. $(2020)$ and Kalami et al. $(2017,2018)$ added additives to their LPF resins to enable them to adhere to standard requirements, while Yaakob et al. (2021) did not specify how the adhesive was made. Therefore, there is potential to optimise the synthesis of lignin formaldehyde resins aiming to replace phenol without requiring any post-modification or additives.

If lignin modification would be required, phenolation is a promising means of increasing the reactivity of lignin as it increases the number of reactive sites and phenolic hydroxyl groups and reduces steric hindrance by 
cleaving weak bonds between units and removing methoxyl groups. Furthermore, a one-pot method has been reported using steam explosion lignin, which allows a direct transition from the phenolation to LPF synthesis reactions in alkaline conditions (Zhao et al., 2016). This one-pot method and phenolation modification could be investigated for the first time, using lignin from the South African pulping industry to determine if this phenolation method would be a viable modification. 


\section{MATERIALS AND METHODOLOGY}

\subsection{Materials}

The two different lignins investigated were a sugarcane bagasse lignin from Sappi Ltd Stanger mill produced using a soda pulping process, and a sodium lignosulphonate from Eucalyptus Grandis (hardwood) obtained from Sappi Ltd Tugela mill, which uses a Neutral Sulphite Semi-Chemical (NSSC) pulping process, using sodium sulphite. The lignins were characterised to determine the properties of the lignin. The sample ID format adopted for the samples was as follows: : (Lignin type) - (Isolation process) - (Purification process) as shown in Table 4 below. The lignin type was denoted 'S' for soda lignin and ' $L$ ' for lignosulphonate. The isolation process was denoted ' $A$ ' for acid precipitation and ' $S$ ' for spray-drying. The purification process was denoted ' $P$ ' for acid precipitation and ' $D$ ' for dialysis. The soda lignin was received in two forms: as a spray-dried black liquor (S-S), and as a black liquor where the lignin was first acid precipitated (S-A) and then acid purified (S-AP). The lignosulphonate sample was received as a spray-dried powder (L-S). The lignosulphonate was purified using a dialysis membrane treatment (L-S-D).

Table 4. Sample IDs for different lignin samples that were used.

\begin{tabular}{llll}
\hline Lignin type & Isolation process & Purification process & ID \\
\hline Soda lignin & Acid precipitated & & S-A \\
Soda lignin & Acid precipitation & Acid purification & S-A-P \\
Phenolated Soda lignin & Phenolation of S-A-P & & P-S-A-P \\
Soda lignin & Spray-dried & & S-S \\
Lignosulphonate & Spray-dried & & L-S \\
Lignosulphonate & Spray-dried & Dialysis & L-S-D \\
\hline
\end{tabular}

Rotary cut pine (Pinus Elliottii) veneer sheets and pine particles were supplied by York Timber based in Mpumalanga, South Africa. The thickness of the veneers varied between one to three millimetres. Bondtite 345 , a commercial tannin-based wood resin, was supplied by Bondtite Pty Ltd., South Africa. Snakeskin dialysis tubing (3500 Da, $35 \mathrm{~mm}$ diameter, $10.5 \mathrm{~m}$ ) were supplied by Thermo Fischer Scientific. The chemicals used in this study were all reagent grade and included the following: Phenol crystals (98\%), Folin-Ciocalteu (FC) phenol reagent, Sodium hydroxide pellets, xylose ( $\geq 99 \%$ ), and vanillin (99\%) was supplied by Sigma Aldrich. Reagent grade sulphuric acid $(\geq 98 \%)$ and formaldehyde (35\%) was supplied by Science World Company SA. Sodium carbonate was supplied by Associated Chemical Enterprises Pty Ltd. Whatman 113 wet strengthened filter paper was used for the filtrations.

\subsection{Experimental design}

\subsubsection{Screening experiments}

Screening experiments were conducted with the S-A-P, S-A, L-S, and L-S-D lignins to see which lignins would be viable to produce LPF resins, aiming for total substitution of phenol by lignin while adhering to the plywood manufacturing standards. The LPF100 resins were synthesised with a NaOH/PL molar ratio of 0.36 , and a F/PL 
molar ratio of 2, according to the method described in section 3.4. From the lignin characterisations (section 4.1) and shear strength screening results (section 4.1.4) it was determined that the S-A-P lignin would be the best suited for use as a phenol substitute/replacement in LPF resins and further optimisation was done only with this resin.

\subsubsection{Face-centred central composite design}

LPF resins were prepared at three different substitution levels: by using either $60 \%, 80 \%$ or $100 \%$ of S-A-P lignin on a weight basis, and the residual phenol. At $60 \%$ and $80 \%$ substitution, a one-pot method (described in section 3.4) was used, where the phenol was used to first improve the lignin reactivity while simultaneously achieving the substitution percentage. A face-centred CCD was used to optimise the shear strength at each substitution level. At $60 \%$ and $80 \%$ substitution the phenolation temperature, $\mathrm{NaOH} / \mathrm{PL}$ ratio, and F/PL ratio was varied as shown in Table 5. At $100 \%$ substitution, only the $\mathrm{NaOH} / \mathrm{PL}$ ratio and $\mathrm{F} / \mathrm{PL}$ ratio was varied, as no phenol was incorporated, as shown in Table 6. These three parameters were chosen for optimisation as they have proven to have a significant effect on the shear strength, as described in section 2.3.2. The ranges of the parameters reported in Table 7 were estimated and chosen as guided by previous reports, which was summarised in Addendum A. Although several parameters affect the shear strength (as discussed in section 2.3.2), the optimisation was limited to these three parameters in this study due to time constraints. The amount of each run was scaled so that it would yield approximately $200 \mathrm{ml}$ of each resin, so that it would fit in the experimental setup and so that enough resin would be available for further characterisation. Four centre point repeats were done for each CCD, which were representative of the standard deviation of the shear strength results of that CCD.

Table 5. The three-factor, three-level CCD used to maximise the shear strength of the LPF60 and LPF80 LPF resins.

\begin{tabular}{cccc}
\hline Run number & $\begin{array}{c}\text { NaOH/PL molar } \\
\text { ratio }\end{array}$ & $\begin{array}{c}\text { F/PL molar } \\
\text { ratio }\end{array}$ & $\begin{array}{c}\text { Temperature } \\
\left({ }^{\circ} \mathbf{C}\right)\end{array}$ \\
\hline 1 & -1 & -1 & -1 \\
2 & -1 & 1 & -1 \\
3 & -1 & 1 & 1 \\
4 & -1 & -1 & 1 \\
5 & 1 & -1 & -1 \\
6 & 1 & 1 & -1 \\
7 & 1 & 1 & 1 \\
8 & 1 & -1 & 1 \\
9 & -1 & 0 & 0 \\
10 & 0 & 1 & 0 \\
11 & 1 & 0 & 0 \\
12 & 0 & -1 & 0 \\
13 & 0 & 0 & -1 \\
14 & 0 & 0 & 1 \\
15 & 0 & 0 & 0 \\
16 & 0 & 0 & 0 \\
17 & 0 & 0 & 0 \\
18 & 0 & 0 & 0 \\
\hline
\end{tabular}

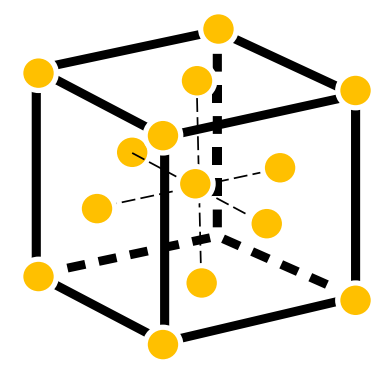


Table 6. The two-factor, three-level CCD used to maximise the shear strength of the LPF100 resins.

\begin{tabular}{ccc}
\hline Run number & NaOH/PL molar ratio & F/PL molar ratio \\
\hline 1 & -1 & -1 \\
2 & -1 & 1 \\
3 & 1 & -1 \\
4 & 1 & 1 \\
5 & -1 & 0 \\
6 & 1 & 0 \\
7 & 0 & 0 \\
8 & 0 & -1 \\
9 & 0 & 1 \\
10 & 0 & 0 \\
11 & 0 & 0 \\
12 & 0 & 0 \\
13 & 0 & 0 \\
\hline
\end{tabular}

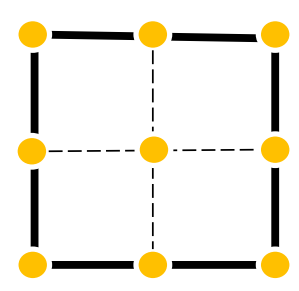

Table 7: The phenolation temperatures, $\mathrm{NaOH} / \mathrm{PL}$ ratios, and F/PL ratios used in the $\mathrm{CCD}$ to maximise the shear strength at each substitution level.

\begin{tabular}{lccc}
\hline & -1 & 0 & 1 \\
\hline $\mathrm{NaOH} / \mathrm{PL}$ molar ratio & 0.12 & 0.36 & 0.63 \\
$\mathrm{~F} / \mathrm{PL}$ molar ratio & 1 & 2 & 3 \\
Temperature $\left({ }^{\circ} \mathrm{C}\right)$ & 80 & 95 & 110 \\
\hline
\end{tabular}

\subsubsection{Statistical analysis}

The analysis of variance (ANOVA) and regression equations were determined from the CCDs using Statistica software version 13.5.0.17. The null hypothesis predicted that all the regression coefficients are equal to zero. If $p<\alpha$, and $F>F_{\text {critical, }}$ the null hypothesis could be rejected, and the parameters were proven to be statistically significant.

\subsection{Lignin isolation and purification methods}

The soda pulping black liquor received was acid precipitated and acid purified to obtain S-A and S-A-P lignins, respectively. It was precipitated through sulphuric acid precipitation, following a method adapted from previous reports (García et al., 2009). The black liquor with an initial pH value of around 12 to 13 was dosed with $98 \%$ sulphuric acid until a pH of 2 was obtained. Approximately $10 \mathrm{~mL} 98 \%$ sulphuric acid was added to $200 \mathrm{~mL}$ of black liquor to obtain a $\mathrm{pH}$ of 2 . The solution was then left for 24 hours for the lignin to precipitate. The lignins were then isolated by centrifugation at $7000 \mathrm{rpm}$ for ten minutes. The recovered lignins were washed twice with distilled water and again placed in a centrifuge at $7000 \mathrm{rpm}$ for ten minutes. The lignins were then air-dried in a fume hood. Thereafter, the lignins were milled into a powder using an Ultra Centrifugal Mill ZM 200 (Retsch) and sieved to a particle size below $425 \mu \mathrm{m}$. The powders were then placed in airtight plastic bags and labelled as S-A lignin until further use.

The S-A lignin was then purified by suspending it in a $1 \mathrm{~N}$ sulphuric acid solution, with a ratio of $200 \mathrm{ml}$ acid solution per gram of dried lignin (García et al., 2009). The solution was left to stand for 24 hours and then 
filtered to separate the lignin solids. The lignin was then washed three times with distilled water to decrease the ash content below five percent. The purified lignins were then air-dried for 24 hours in a fume hood, then placed in airtight plastic bags and labelled as S-A-P until further use. The yield of the acid purification process was around $70 \%$. Ten litres of black liquor were acid precipitated and acid purified to give a total of around 2.5 $\mathrm{kg} \mathrm{S-A-P} \mathrm{lignin} \mathrm{that} \mathrm{was} \mathrm{required} \mathrm{for} \mathrm{the} \mathrm{LPF} \mathrm{synthesis} \mathrm{and} \mathrm{characterisations} \mathrm{performed.}$

Another soda lignin sample was supplied by the sponsor where the black liquor was spray-dried and received in powder form. This lignin sample was not further processed but used as received and labelled as S-S.

Sodium lignosulphonate was received as a spray-dried powder (L-S) and had a high degree of impurities and was therefore purified by a dialysis membrane. Lignosulphonate has a high molecular weight, where the aim was to retain the lignosulphonate in the dialysis membrane, while therefore the hemicelluloses, simple sugars, and inorganic contaminants present were to be removed through the membrane to the buffer. The molecular weight of the lignosulphonate samples could not be analysed (for example by GPC), due to their high impurity content that could damage equipment. Therefore, the MWCO of the dialysis membrane was chosen after talking to suppliers and referring to previous reports using MWCOs of 2000 and $5000 \mathrm{Da}$ (Marques et al., 2009) and an MWCO of 3500 Da (Angelini et al., 2019) for lignosulphonate purifications. Dialysis was performed following the instructions from the supplier. The sodium lignosulphonate powder was dissolved in distilled water to make a $25 \mathrm{wt} \%$ solution. The solution was then poured into the dialysis tubing $(3500 \mathrm{Da}, 35 \mathrm{~mm}$ diameter), which was placed in a plastic bucket with a distilled water buffer to dialysis solution volume ratio of 20. Three buffer exchanges were performed: after four hours, after another four hours, and then it was left for another twelve hours. The solution was then poured into tinfoil trays and dried for 24 hours in a $40{ }^{\circ} \mathrm{C}$ oven and then stored and labelled as L-S-D.

\subsection{One-pot phenolation and LPF resin synthesis}

A one-pot method was used based on that reported by (Zhao et al., 2016). The setup consisted of a five-neck round bottom flask which was placed in a heating mantle and equipped with a laboratory-scale overhead digital stirrer, dropping funnel, thermometer, flange lid, and a reflux condenser. The phenolation reaction was performed by weighing the required amount of phenol and lignin (sum of fifty grams) into the flask. Two-thirds of the required amount of the $1 \mathrm{M} \mathrm{NaOH}$ solution (as done by Kalami et al., (2017)) was then added to the flask and it was mixed until all the phenol and lignin had dissolved. The temperature was slowly increased to the phenolation temperature in agreement with the CCD run. Once the temperature was reached, the phenolation reaction was left to occur for 1.5 hours, with continuous stirring. When the reaction was complete, the mixture was left to cool to a temperature below $40{ }^{\circ} \mathrm{C}$.

With the phenolation step complete, the addition reaction was started. The required amount of formaldehyde was added dropwise using a dropping funnel to prevent runaway reactions, whilst stirring continuously. The temperature was monitored as the reaction is exothermic. If the temperature increased a lot, the formaldehyde addition was to be stopped, and the mixture was placed in a water bath to prevent a runaway reaction. Once all the formaldehyde was added, the temperature was slowly increased to $65^{\circ} \mathrm{C}$ and allowed to react for ten minutes for the addition reaction to occur. 


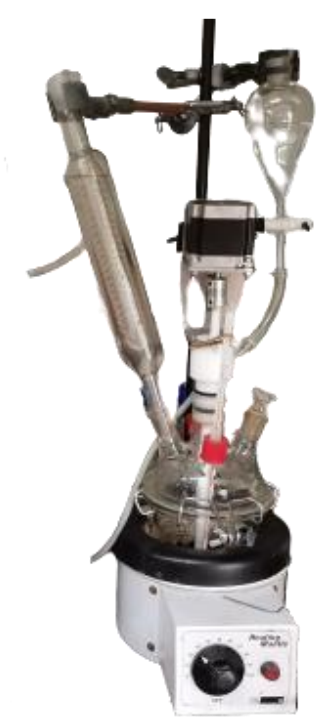

Figure 6. Experimental setup for one-pot phenolation and LPF synthesis.

Thereafter, the condensation reaction was then initiated. The last third of $1 \mathrm{M} \mathrm{NaOH}$ was added via the dropping funnel, while the temperature was slowly increased to $85^{\circ} \mathrm{C}$. Once the temperature was reached, the reaction was allowed to continue for one hour. The mixture was left to cool to room temperature. The obtained resin was stored in the freezer at $-8{ }^{\circ} \mathrm{C}$ to prevent further polymerisation reactions from occurring. The resins were then later poured into tinfoil trays and placed in a vacuum oven for drying. The vacuum oven was maintained at a temperature of $45{ }^{\circ} \mathrm{C}$ and continuously purged with nitrogen gas to prevent the accumulation of unreacted phenol and formaldehyde gasses in the system. The dried resins were then milled using an Ultra Centrifugal Mill ZM 200 (Retsch) into powders with a particle size below $0.5 \mathrm{~mm}$. They were stored in airtight plastic bags as these alkaline resoles are hygroscopic, to prevent the capture of sodium carbonate or carbon dioxide which could lead to the formation of ash (Lorenz \& Christiansen, 1995). This procedure was followed for every run in the CCD and replicated four times at centre point conditions.

\subsection{Characterisation of the lignins and LPF resins}

\subsubsection{Proximate analysis of the lignins}

Proximate analyses were performed on the lignin samples according to methods described in NREL/TP-51042624 and NREL/TP-510-42622 standards, to determine the moisture and ash content, respectively. When determining the moisture content, clean watch glasses were pre-dried in a $105^{\circ} \mathrm{C}$ oven for at least four hours. They were then placed in a desiccator to cool, whereafter the weight of each watch glass was recorded. Approximately two grams of a sample was loaded onto a watch glass and the weight was recorded. The watch glasses were then returned to the oven and left for 24 hours. The watch glasses were then removed from the oven and cooled in a desiccator to room temperature, whereafter the weight was recorded again. To ensure constant weight, the samples were then returned to the oven for an hour, whereafter they were again cooled in a desiccator and weighed. All samples were analysed in triplicate. The moisture content could then be calculated according to Equation 1. 
Equation 1:

$$
\text { Moisture content }=\left(\frac{\text { weight sample }- \text { weight dried sample }}{\text { weight sample }}\right)
$$

When determining the ash content, clean crucibles were placed in a $105^{\circ} \mathrm{C}$ oven and pre-dried for 24 hours. They were then cooled in a desiccator and the weight of each crucible was then recorded. Approximately 1.5 $\mathrm{g}$ of each sample was then loaded into a crucible, and the weight of each was recorded. The soda lignin samples were placed into a muffle furnace, which was gradually heated over 45 minutes to a temperature of $575{ }^{\circ} \mathrm{C}$, and held at that temperature for four hours. The soda lignin samples were then removed, cooled in a desiccator, and then the weight of each was recorded. The ash content of the lignosulphonate samples was determined similarly but using a muffle furnace with a ramping program that could attain a higher temperature. Firstly, the furnace was heated to $250{ }^{\circ} \mathrm{C}$ over 25 minutes and held at that temperature for 30 minutes. Secondly, the temperature was then increased to $800^{\circ} \mathrm{C}$ over 40 minutes and held there for 16 hours. Afterwards, the furnace was cooled to $200^{\circ} \mathrm{C}$, then the crucibles were removed and placed in a desiccator to cool down. The weight of each crucible was then recorded, and the ash content was determined according to Equation 2.

Equation 2:

$$
\text { Ash content }=1-\left(\frac{\text { weight ashed sample }}{\text { weight sample }}\right)
$$

\subsubsection{Phenolic hydroxyl content determination of the lignins and LPF resins}

Phenolic hydroxyl groups are the most reactive functional groups in the polymerisation reaction during LPF synthesis with formaldehyde, therefore they are an indication of the reactivity of the lignin. A high concentration of phenolic hydroxyl groups is thus favourable (Zhang et al., 2013; Laurichesse \& Avérous, 2014). The phenolic hydroxyl group content of the lignins and the LPF resins were determined following a method described by Areskogh ( 2010) to estimate the reactivity of the lignin and phenolated lignin. Soda lignin and phenolated soda lignin samples were prepared by dissolving $0.05 \mathrm{~g}$ per litre of $0.05 \mathrm{M} \mathrm{NaOH}$ solution, while lignosulphonate samples were prepared by dissolving $0.05 \mathrm{~g}$ per litre distilled water. A blank sample was also prepared as a reference for the $\mathrm{NaOH}$ and distilled water solutions. For each sample, approximately $30 \mathrm{~mm}$ of distilled water was added to a beaker, and $3 \mathrm{ml}$ of FC reagent and $1 \mathrm{ml}$ of the sample was added. The mixture was left for eight minutes, whereafter ten millilitres of a twenty-mass percentage sodium carbonate solution was added. The mixture was poured into a fifty-millilitre volumetric flask and topped up with distilled water to the fifty-millilitre mark. The solution was then placed in a beaker again and mixed in an incubator for two hours. The absorbance was measured at 760 nm using a UV spectrophotometer (CECIL 2021 AQUARIUS 2000 series, 190 to $1000 \mathrm{~nm}$ ).

A calibration curve (Figure 7) was set up by determining absorbances of standard solutions, to obtain a linear correlation. Vanillin was used as the standard solution at a concentration range from 0 to $5 \mathrm{mmol}$ per litre. The absorbance of the lignin samples was then converted to concentration using this linear correlation and then divided by the lignin content in the sample $(0.5 \mathrm{~g} / \mathrm{L})$ to obtain the amount of millimole phenolic hydroxyls 
present per gram of the sample. The same procedure was repeated with the phenolated lignin samples to verify if the phenolation was successful and to correlate the phenolic hydroxyl content with the shear strength obtained.

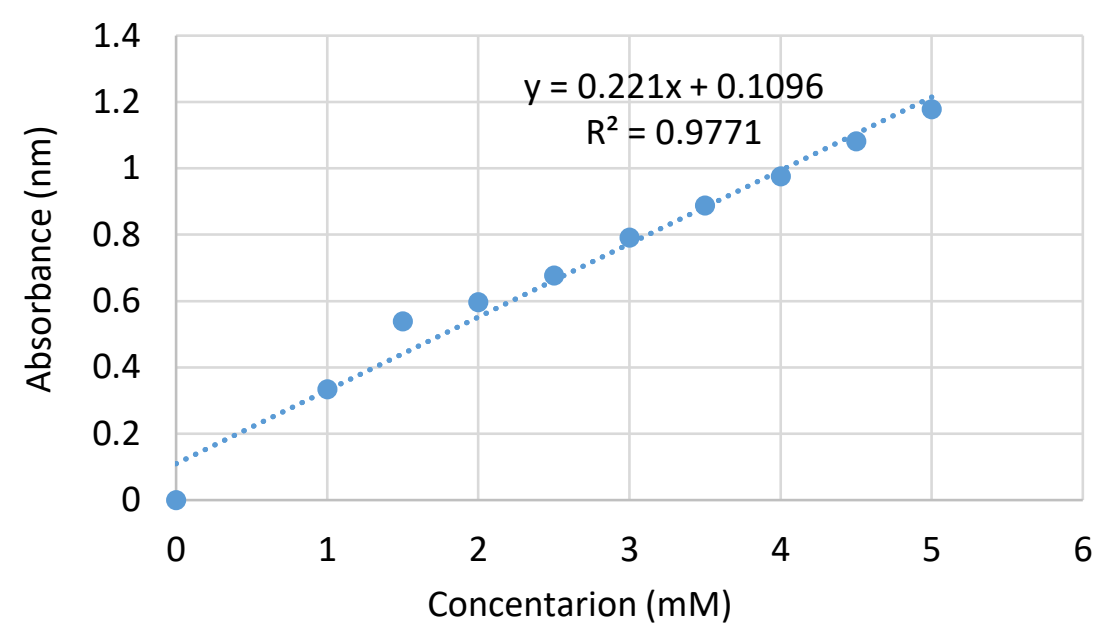

Figure 7. Calibration curve for converting concentration of phenolic hydroxyl groups to absorbance.

\subsubsection{Elemental analysis of the lignins}

The Central Analytical Facility at Stellenbosch University conducted the elemental analysis of the lignin samples using an Elementar Vario EL Cube Elemental Analyser, Frankfurt Germany. This characterisation aimed to determine if dialysis was successful in removing the residual sulphur from the lignosulphonates and to see if sulphur was present after acid purification and acid precipitation of the soda lignin. Dry combustion was used to determine the $\mathrm{C}: \mathrm{H}: \mathrm{N}: \mathrm{S}$ ratios of the S-A-P, L-S and L-S-D samples. The samples were weighed and placed on an aluminium foil boat along with $5 \mathrm{mg}$ of tungsten trioxide powder. It was then introduced using an autosampler and combusted at $1050{ }^{\circ} \mathrm{C}$ in an oxygen-enriched column at two bars. Argon carrier gas at around one bar was used to transfer the gaseous product through a reduction tube with a copper wire at a temperature of $850{ }^{\circ} \mathrm{C}$. The thermal conductivity detector was set at $60^{\circ} \mathrm{C}$ with the argon flow rate at 220 $\mathrm{ml} / \mathrm{min}$. A certified reference material was used to ensure accuracy of the results obtained. The elimination water, carbon dioxide, sulphur dioxide, sulphur trioxide, and $\mathrm{NO}_{\mathrm{x}}$, was used to determine the amounts of $\mathrm{C}, \mathrm{H}$, $\mathrm{N}$, and $\mathrm{S}$ present. The oxygen content was determined as the remaining portion of the sample, after correcting for the ash and moisture contents.

\subsubsection{Thermogravimetric analysis of the lignins and LPF resins}

A TGA Q50 thermogravimetric apparatus was used to determine the thermal decomposition behaviour of the different lignin samples and some of the LPF resin samples. Approximately $5 \pm 0.5 \mathrm{mg}$ of sample was loaded onto an aluminium crucible which was placed in a TGA pan. A heating rate of $10^{\circ} \mathrm{C} / \mathrm{min}$ was used to heat the sample from around $20^{\circ} \mathrm{C}$ up to $595^{\circ} \mathrm{C}$ under nitrogen atmosphere (Govender, Majeke, et al., 2020). The data obtained were then used to construct plots of weight loss against temperature, and degradation curves from the first derivative thereof, using TRIOS software. This analysis was done to indicate the degree of cross-linking 
that occurred in the LPF resins, as a high thermal stability indicates the presence of strong bonds and large cross-linked networks formed, which could then lead to high shear strengths.

\subsubsection{Mid-IR spectroscopy analysis of the lignins and LPF resins}

The lignin samples and some of the LPF resin samples were characterised using mid-IR spectroscopy analysis. Specific LPF resins were analysed at each substitution level, which included the centre point, as well as two good and two bad performing samples identified. The aim was to identify chemical and structural differences in the resins and the effect they would have on the shear strength. The analysis was performed with samples in powdered form, which was pressed against the diamond crystal surface, using a Bruker Alpha P ATR-IR instrument. The Fourier transform infrared spectrometer operated in Attenuated Total Reflectance mode at a resolution of $4 \mathrm{~cm}^{-1}$, with 32 scans per sample within the mid-IR absorption band region of 4000 to $400 \mathrm{~cm}^{-1}$ (Govender, Majeke, et al., 2020). A background measurement was performed before each experimental spectra was recorded. Wiley Knowitall Spectroscopy Edition 2020 software was used to plot and analyse the spectra. The spectral data were baseline-corrected and normalised to one for comparison with each other. The absorption bands were allocated using previous reports. The spectra were then compared in a principal component analysis (PCA) which was performed using Statistica software version 13.5.0.17.

\subsubsection{Free formaldehyde content determination of the LPF resins}

The free formaldehyde content of the LPF resins was determined according to the European Standard DIN EN ISO 9397 (Kalami et al., 2018). Industrial implementation of LPF resins would need to have a low free formaldehyde content as it could cause toxic emissions. Potentiometric titration was performed to quantify the amount of $\mathrm{HCl}$ released during the reaction as shown in Equation 3, where hydroxylamine hydrochloride $\left(\mathrm{NH}_{2} \mathrm{OH} \mathrm{HCl}\right)$ reacts with formaldehyde $\left(\mathrm{CH}_{2} \mathrm{O}\right)$ to form formaldoxime $\left(\mathrm{CH}_{2}=\mathrm{NOH}\right)$. One hundred millilitres of distilled water was added to approximately two grams of resin, and the $\mathrm{pH}$ of the solution was adjusted to a value of four, using a $0.1 \mathrm{~N}$ hydrochloric acid solution. Twenty millilitres of a ten percent hydroxylamine hydrochloride solution was then added to the resins, whereafter the solution was titrated using a $0.1 \mathrm{~N}$ sodium hydroxide solution to a $\mathrm{pH}$ value of four again. The percentage of free formaldehyde was calculated using Equation 4.

Equation 3:

$$
\mathrm{HCHO}+\mathrm{NH}_{2} \mathrm{OH} \mathrm{HCl} \rightarrow \mathrm{CH}_{2}=\mathrm{NOH}+\mathrm{H}_{2} \mathrm{O}+\mathrm{HCl}
$$

Equation 4:

$$
\% \mathrm{CH}_{2} \mathrm{O}=\frac{V_{\mathrm{NaOH}} \times N_{\mathrm{NaOH}} \times 3.003}{m_{\text {resin }}}
$$

\subsubsection{Plywood preparation and shear strength tests}

Adhesives were prepared by mixing the LPF resin powder with deionised water so that the powders are dissolved or in suspension and could be applied and spread onto the plywood. The lignosulphonates are soluble in water; therefore, they required less water to dissolve, than the soda lignin required to be in suspension. The mass ratio of the lignosulphonate resin to water used was 60:40, while the soda lignin resin 
to water mass ratio used was 45:55. Rotary cut pine veneers were used to make three-layered plywood boards with a thickness around $6 \mathrm{~mm}$ after pressing. The boards were made according to the ASTM D906-98 (2017) standard. The plywood was conditioned around $22{ }^{\circ} \mathrm{C}$ and $60 \%$ relative humidity for seven days (Alawode et al., 2019). The veneers were cut into $100 \mathrm{~mm} \times 60 \mathrm{~mm}$ boards, with a thickness varying between 1 to $3 \mathrm{~mm}$. The adhesive was then applied with a spread rate of $400 \mathrm{~g} / \mathrm{m}^{2}$ on the surfaces of the two outer veneers and left for ten minutes to evaporate the excessive moisture. The boards were then assembled with the grain direction of the face and back plies in the vertical (strong) direction, and the grain direction of the middle layer perpendicular to that of the outer plies. Each board was then hot-pressed individually for ten minutes at 160 ${ }^{\circ} \mathrm{C}$ and $1.6 \mathrm{MPa}$. The boards were left to cure in a conditioning room for seven days at $22{ }^{\circ} \mathrm{C}$ and $60 \%$ relative humidity before shear strength testing.

Each plywood board was cut after conditioning into an $82.6 \mathrm{~mm}$ by $25.4 \mathrm{~mm}$ specimen, to remove the outer regions where the adhesives were not well spread. The specimen was cut $2 / 3$ through the core of the front and middle plane at $25.4 \mathrm{~mm}$ from the top, as well as $2 / 3$ through the core of the back and middle plain 25.4 $\mathrm{mm}$ from the bottom of the specimen. Thus, when the specimen is placed in tension by pulling the top and bottom, the shear strength of the adhesive was tested. The boards were tested in an MTS Criterion Model 43 testing machine. The crosshead speed was set to $7 \mathrm{~mm} / \mathrm{min}$ to obtain a loading rate between 4535 and 7560 $\mathrm{g} / \mathrm{s}$ as specified by the ASTM D 906-98 (2017) standard. The shear strength could then be calculated by dividing the maximum load obtained, by the surface area.

\subsubsection{Performance valuation of the resin in particleboard manufacture}

Particleboard was produced with the LPF68 resin and Bondtite as a reference. The aim was to confirm the good adhesive strength properties seen in plywood applications for particleboard applications also. Furthermore, this also allowed the evaluation of the moisture resistance of the resin. Pine particles were milled and sieved to a size smaller than one millimetre and conditioned at $22{ }^{\circ} \mathrm{C}$ and $60 \%$ relative humidity for 96 hours. The equilibrium moisture content of the particles was determined to be $10 \%$. The resin and pine particles were mixed manually and poured into a steel mould $(300 \times 300 \mathrm{~mm})$, with a $27 \mathrm{~mm}$ thick steel plate placed on top to compress the composite in the mould. The boards were pressed at $165^{\circ} \mathrm{C}$ and $20 \mathrm{MPa}$ for 10 minutes (Alawode et al., 2019). The boards were then removed from the mould and conditioned in a conditioning room at $22{ }^{\circ} \mathrm{C}$ and $60 \%$ relative humidity for 96 hours before testing. The amount of resin used was $10 \%$, whereof the solid content of the resin was $48 \%$.

The properties of the particleboards were then tested to quantify the modulus of rupture (MOR), modulus of elasticity (MOE), thickness swelling (TS), and water absorption (WA). The particleboards were tested according to the ASTM D1037-12 standard, using an Instron testing machine equipped with a $5 \mathrm{kN}$ load cell. The boards were cut using a vertical bandsaw into $195 \times 51 \mathrm{~mm}$ boards, as specified by the standard for mechanical testing, and tests were performed with four replicates for each resin. A load was applied to the boards at a loading rate of $3 \mathrm{~mm} / \mathrm{min}$, while the load and deflection were recorded for each sample with an accuracy of $\pm 0.5 \%$ of the reading. After mechanical testing, the boards were cut again into dimensions of $85 \times 51 \mathrm{~mm}$ for dimensional stability, using six replicates for each resin. The dimensions and weight of the boards were then recorded to determine the WA and TS of the boards. They were then submerged horizontally in distilled water 
for 24 hours, whereafter the excess water was drained before they were weighed and measured again. The moisture content $(\mathrm{MC})$ of the boards were determined by drying the boards in an oven at $103{ }^{\circ} \mathrm{C}$ after submersion. The MOR, MOE, TS and WA were calculated with the equations as specified in the ASTM D1037- 12 standard. 


\section{RESULTS AND DISCUSSION}

\subsection{Lignin characterisation}

\subsubsection{Structural composition analysis}

Of the lignins investigated, S-A-P had the lowest ash content, which foreshadows a high reactivity with formaldehyde and is desirable for LPF resin applications. The spray-dried samples indicated a high ash content compared to the samples that were acid precipitated, as seen in Table 8. S-S had a very high ash content of $62.6 \%$. The ash content in L-S was halved after dialysis from $24.8 \%$ only to $12.8 \%$. The ash content of $14.9 \%$ present after acid precipitation (S-A) was significantly reduced to $2.16 \%$ after acid purification (S-A-P). The ash contents agreed with previous reports where native lignin from grasses generally have ash contents from two to five percent, while wood-derived lignin has ash contents below one percent (Chen, 2014). The high ash content in S-S was expected, as it was soda black liquor that was spray-dried and still contained pulping chemicals, such as $\mathrm{Na}^{+}$. The residual $\mathrm{NaOH}$ and $\mathrm{Na}_{2} \mathrm{CO}_{3}$ were removed during the acid precipitation and acid purification stages. The higher ash in L-S was also expected as it was not purified, and during sulphite pulping sulphur dioxide was used which contributed to the high ash content of the L-S sample. $51.6 \%$ of the ash was removed during dialysis which indicated that inorganics were attached to the lignin structure, such as sulphurcontaining groups. High ash contents were undesirable as they could cause a catalytic effect which could make lignin degrade at lower temperatures (Naron et al., 2017), and they could cause undesirable side reactions. Agglomeration of ash components could have also occurred which promotes clustering and could prevent LPF resins from dispersing into the plywood for effective bonding.

Table 8. Compositions of lignin samples in terms of moisture, ash, organic, and phenolic $\mathrm{OH}$ contents (Standard deviations reported in brackets).

\begin{tabular}{lccccc}
\hline & $\begin{array}{c}\text { Moisture } \\
\text { content (wt\%) }\end{array}$ & $\begin{array}{c}\text { Ash content } \\
(\mathbf{w t} \%)\end{array}$ & $\begin{array}{c}\text { Organic } \\
\text { content (wt\%) }\end{array}$ & $\begin{array}{c}\text { Phenolic OH } \\
\text { (wt\% of organic \%) }\end{array}$ & $\begin{array}{c}\text { Phenolic OH } \\
\text { (mmol/g sample) }\end{array}$ \\
\hline S-A & $9.63(0.75)$ & $14.9(0.41)$ & 75.5 & 2.91 & $1.05(0.34)$ \\
S-A-P & $13.8(0.51)$ & $2.16(0.01)$ & 84.0 & 3.56 & $1.70(0.54)$ \\
S-S & $2.86(0.07)$ & $59.7(1.14)$ & 34.5 & $\mathrm{ND}^{1}$ & $\mathrm{ND}^{1}$ \\
L-S & $4.01(0.22)$ & $24.8(1.10)$ & 71.2 & 3.65 & $1.53(0.45)$ \\
L-S-D & $10.4(0.18)$ & $12.8(0.37)$ & 76.8 & 3.28 & $1.48(0.28)$ \\
Phenol & $\mathrm{ND}^{1}$ & $\mathrm{ND}^{1}$ & $\mathrm{ND}^{1}$ & 16.9 & $9.91(0.10)$ \\
\hline
\end{tabular}

${ }^{1}$ ND - Not determined.

The phenolic hydroxyl groups are very reactive functional groups with free electrons on the oxygen that stabilize the intermediate cation causing a high reactivity toward electrophilic aromatic substitutions on the ortho- or para-positions (Hu et al., 2011). to make the lignin reactive with formaldehyde in the synthesis reaction to produce LPF resins (Abdelwahab \& Nassar, 2011; Doherty et al., 2011). The phenolic hydroxyl contents reported in Table 8 were re-expressed as weight percentages of the organic content so that they could be compared while correcting for the effect of the moisture and ash contents. The phenolic hydroxyl 
content of the S-A was $2.91 \%$ and increased to $3.56 \%$ in S-A-P. The phenolic hydroxyl content of L-S was 3.65\% and reduced after dialysis to $3.28 \%$, which was attributed to the loss of phenolic hydroxyl groups on the $\mathrm{H}$ and G-units of the lignin through the dialysis membrane. The phenolic hydroxyl group contents of S-A-P and L-S were similar and corresponded to previous reports that used the same quantification method (Matsushita, 2015; Podschun, Stücker, et al., 2015; Kalami et al., 2018; Taverna et al., 2019). The phenolic hydroxyl content of phenol was determined to be $9.91 \mathrm{mmol} / \mathrm{g}$ sample, which was high in comparison to the lignin samples and corresponded to previous reports (Zhao et al., 2016). Severe pulping conditions cause pulping lignins to have a recalcitrant and inert structure that is less reactive than natural lignin, while other processes (such as organosolv) lead to a structure closer to natural lignin (Karthäuser et al., 2021).

The elemental analysis results were consistent with values reported previously for similar lignin samples (Zhou et al., 2016; Naron et al., 2017) and was performed to compare the soda lignin to the lignosulphonate, and to determine if dialysis removed sulphur present. From the results in Table 9, it was seen that the carbon and hydrogen contents in S-A-P were higher than that of the lignosulphonates, which was expected as the lignosulphonates had a significantly higher ash content. The amount of carbon and hydrogen in L-S proportionally increased in L-S-D, as some of the inorganics were removed. All the samples had trace amounts of nitrogen, which was attributed to the biomass sources. The S-A-P sample was sulphur-free, which indicated that no significant amount of the sulphur from the sulphuric acid precipitation or purification remained. The lignosulphonate samples contained sulphur which remained from the sulphite pulping process. The sulphur content of L-S decreased from $7.5 \%$ to $5.6 \%$ in L-S-D after dialysis, but not all was removed as during sulphite pulping the sulphur attached as sulphonate groups onto the lignin structure. This incorporation of anionic groups (such as sulphonate, carboxylate and phenolic hydroxyl groups) rendered the lignosulphonates hydrophilic (Amiandamhen, Meincken \& Tyhoda, 2018), which presented challenges in the drying stages for LPF resin production.

Table 9. Elemental analysis results of the lignin samples.

\begin{tabular}{lccccc}
\hline & $\mathbf{C ~ ( \% )}$ & $\mathbf{H ~ ( \% )}$ & $\mathbf{N ~ ( \% )}$ & $\mathbf{S ~ ( \% ) ~}$ & $\mathbf{O}(\%)$ \\
\hline S-A-P & 53.4 & 7.1 & 0.28 & $\mathrm{BDL}^{1}$ & 23.2 \\
L-S & 35.4 & 4.7 & 0.19 & 7.5 & 23.5 \\
L-S-D & 41.7 & 6.0 & 0.26 & 5.6 & 23.2 \\
\hline
\end{tabular}

${ }^{1} \mathrm{BDL}$ - below detection limit

\subsubsection{Mid-IR spectra of the lignin and lignosulphonate samples}

The mid-IR spectral analysis was used to determine and compare the chemical structures of the lignin samples. The spectral region of the lignin structure $\left(4000\right.$ to $\left.600 \mathrm{~cm}^{-1}\right)$ is shown in Figure 8, while only the "fingerprint" region (1500 to $600 \mathrm{~cm}^{-1}$ ) is shown in Figure 9. The samples were analysed in duplicate. The spectra and the main peaks observed of the lignin samples corresponded to previous reports. The peak assignments as previously reported are summarised in Table 10 (Alonso et al., 2001; Hu et al., 2012; Moubarik et al., 2013; Zhao et al., 2016; Hussin et al., 2017; El Mansouri, Qiaolong \& Huang, 2018). 


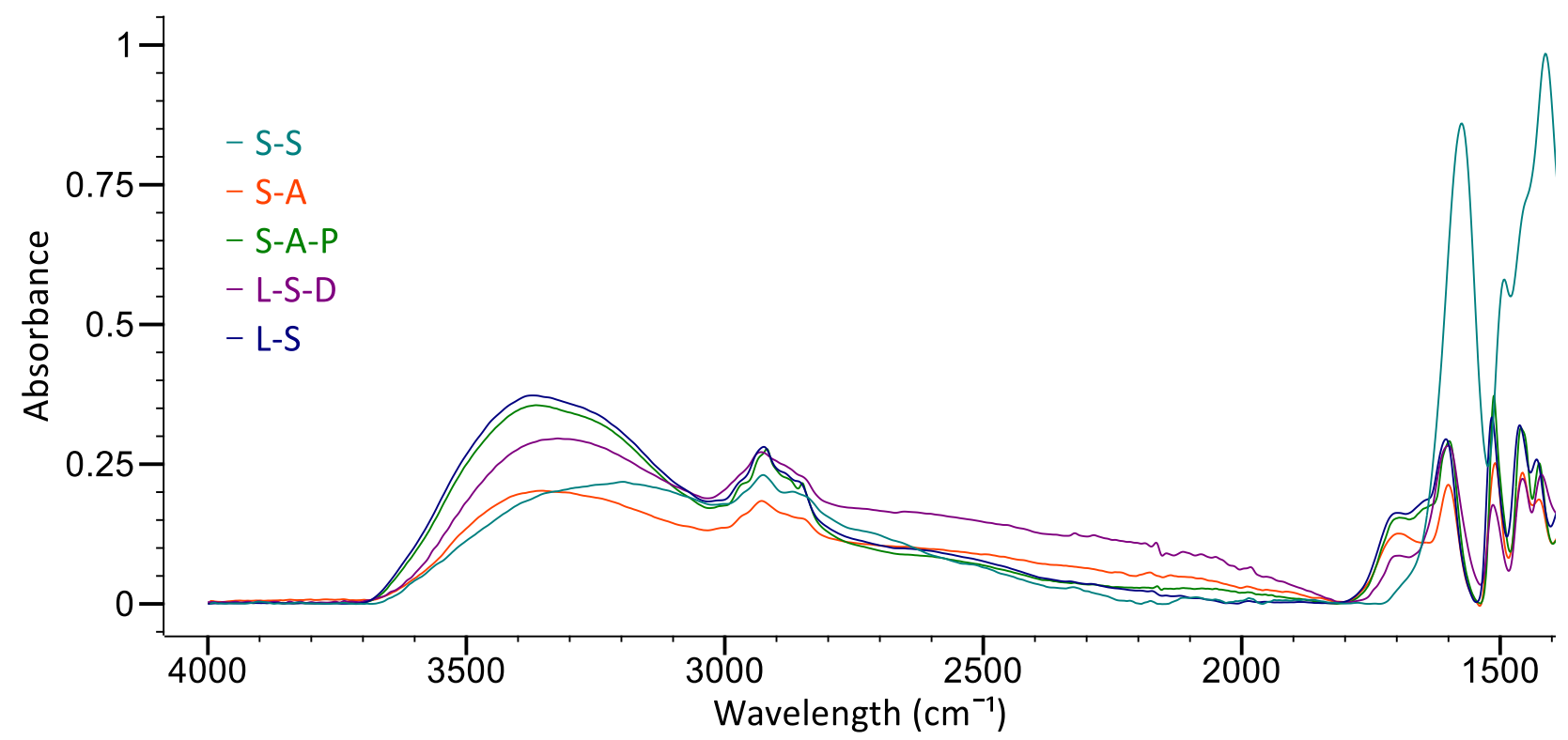

Figure 8. Mid-IR spectra of the different lignin samples in the single (4000 to $2500 \mathrm{~cm}^{-1}$ ), triple (2500 to $\left.2000 \mathrm{~cm}^{-1}\right)$, and double bond (2000 to $1500 \mathrm{~cm}^{-1}$ ) regions.

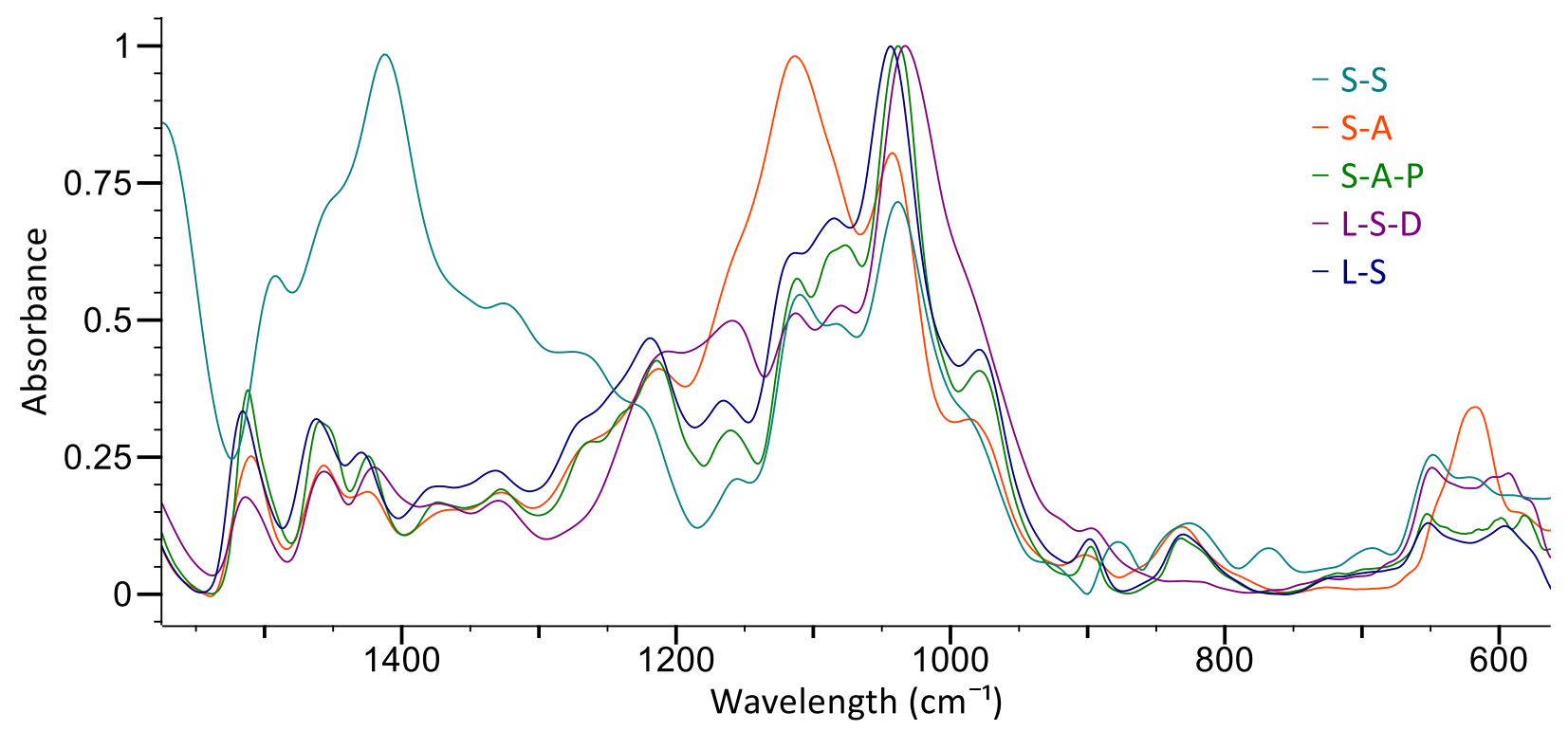

Figure 9: Mid-IR spectra of the different lignin samples in the fingerprint region (1500 to $600 \mathrm{~cm}^{-1}$ ).

All the samples showed similar wide peaks at 3400 to $3200 \mathrm{~cm}^{-1}$, except S-S which peaked at $3197 \mathrm{~cm}^{-1}$, which represented the total hydroxyl stretching vibrations in aromatic and aliphatic structures. The high peak for total hydroxyls here, corresponded with the higher phenolic hydroxyl contents of L-S and S-A-P observed in Table 8. All the lignins had peaks at 2925 and $2848 \mathrm{~cm}^{-1}$ which were attributed to either lignin or polysaccharides, representing the $\mathrm{C}-\mathrm{H}$ vibrations in the methyl $\left(\mathrm{CH}_{3}\right)$ and methylene $\left(\mathrm{CH}_{2}\right)$ groups, respectively. All showed shoulder bands for unconjugated ketones at $1695 \mathrm{~cm}^{-1}$, except the S-S sample which showed no shoulder here. The absence of this peak in S-S was attributed to the potential cleavage of $\beta$-glycosidic ethyl linkages between hemicellulose and lignin during the acid precipitation. This was confirmed by the absence of a peak at $693 \mathrm{~cm}^{-1}$ in S-A-P attributed to $\beta$-glycosidic ethyl linkages between lignin and hemicellulose (Mou, Li \& Fardim, 2014). 
Table 10: Peak assignments for the mid-IR spectra as from previous reports.

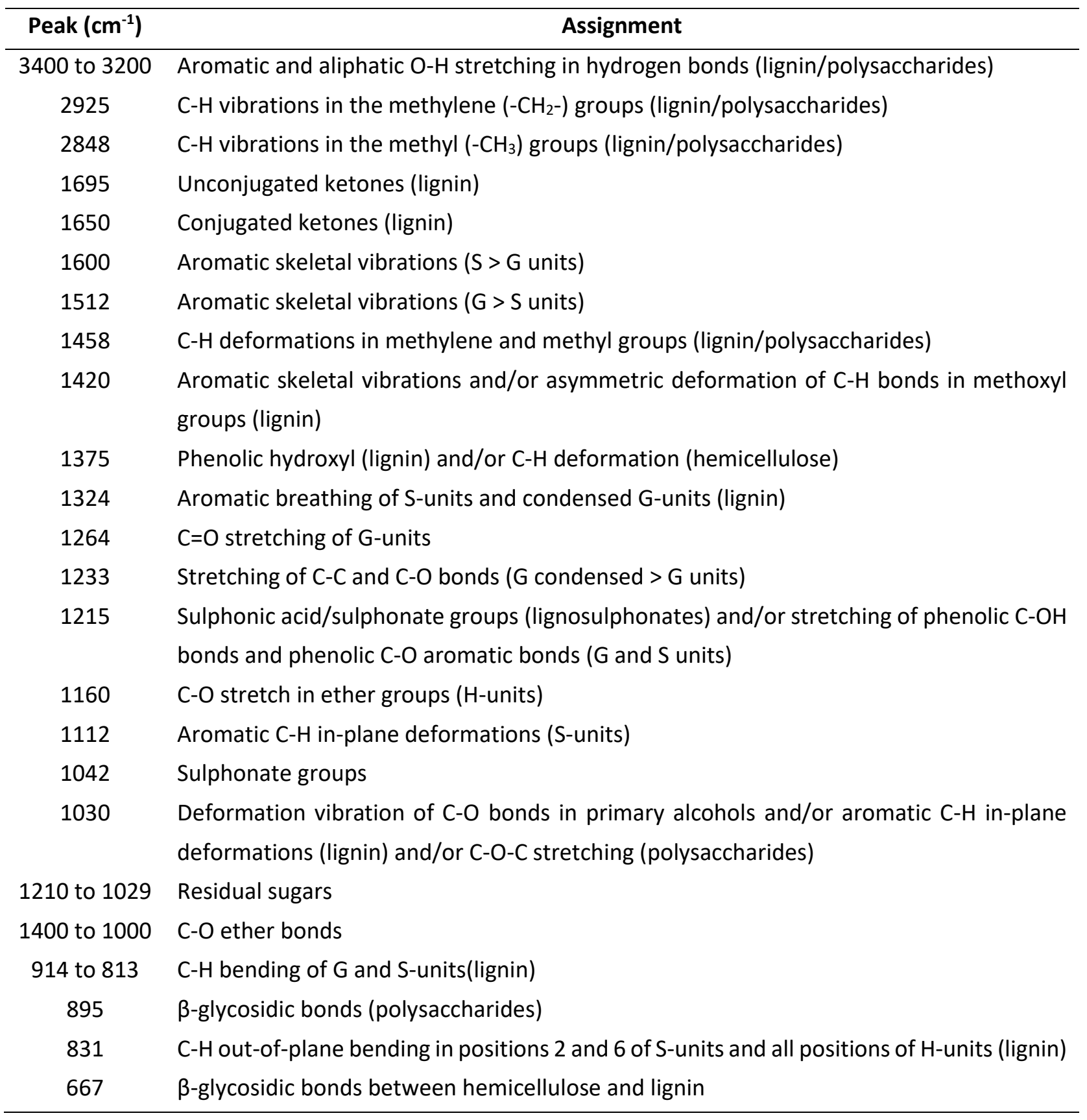

The band at $1375 \mathrm{~cm}^{-1}$ represented the phenolic hydroxyl bonds in lignin, and/or the $\mathrm{C}-\mathrm{H}$ deformation in hemicellulose. This peak was absent in S-S, which was attributed to its low purity, and its high pH value of the black liquor, where phenolates were not converted to phenolic hydroxyl groups as during acid precipitation. The peak at $1215 \mathrm{~cm}^{-1}$ was characteristic of sulphonic acid or sulphonate groups in the lignosulphonates, and/or attributed to stretching of phenolic $\mathrm{C}-\mathrm{OH}$ bonds and phenolic $\mathrm{C}-\mathrm{O}$ aromatic bonds in $\mathrm{G}$ - or S-units. There was no peak for S-S. The lignosulphonate samples had a broader peak here, indicating sulphonic acid/sulphonate groups. It could also be possible that metal impurities (such as sodium) in the lignosulphonates could have acted as counterions leading to peak shifts of the $\mathrm{C}-\mathrm{O}$ stretching band. 
Aromatic skeletal vibrations of S- and G-units occurred from 1600 to $1500 \mathrm{~cm}^{-1}$. At 1600 to $1570 \mathrm{~cm}^{-1}$ the Sunits was more abundant than the G-units, while at 1520 to $1495 \mathrm{~cm}^{-1}$ the G-units were more abundant than the S-units. L-S-D indicated more S-units than L-S, which could be attributed to the removal of G-and H-units during dialysis. The S-S sample also indicated more S-units, which was attributed to the acid precipitation process that could have cleaved the $\beta-0-4$ ether bonds between the G- and/or S-units in S-A and S-A-P. Aromatic skeletal vibrations and/or asymmetric deformations of $\mathrm{C}-\mathrm{H}$ bonds in methoxyl groups in lignin occurred at $1420 \mathrm{~cm}^{-1}$. These peaks were higher for the S-S and L-S-D lignins, which confirmed that they had more G- or S-units than the other lignins. At $1160 \mathrm{~cm}^{-1}$ only the S-A-P, L-S and L-S-D samples had peaks, which was attributed to $\mathrm{C}-\mathrm{O}$ stretching in ether groups in the $\mathrm{H}$-units present (Moubarik et al., 2013). Bagasse lignin is known to contain appreciable amounts of all three units, while hardwoods have the most S-units, a lot of Gunits, and only a small amount of H-units (Gellerstedt et al., 2009). The L-S sample showed the presence of Hunits, which was unexpected for hardwoods. However, it could be a result of the sulphite pulping conditions which could have reduced the methoxyl groups at the ortho-positions on the aromatic ring. The decrease in the H-units in L-S-D confirmed that some of the lower molecular weight units of the lignin were removed through the membrane during dialysis. The peak at $1460 \mathrm{~cm}^{-1}$ corresponded to the $\mathrm{C}-\mathrm{H}$ asymmetric vibrations and deformations in methyl and/or methylene groups of lignin or polysaccharides still present from the native lignin structure (Nadji et al., 2009). A decrease in these peaks was observed for L-S-D and could indicate that lignin or polysaccharides were removed during dialysis. The band at $895 \mathrm{~cm}^{-1}$ resulted from $\beta$-glycosidic bonds between monosaccharides, where the absence of a peak in the L-S-D sample indicated that some of the sugars were removed during dialysis.

\subsubsection{PCA of the lignin and lignosulphonate samples}

The principal components (PC) that described most of the variability in the data were: $P C 1, P C 2, P C 3$, and PC4 which described $61.3 \%, 16.1 \%, 9.2 \%$ and $4.4 \%$, respectively, as shown in Figure 10. The PCA plots of the mid-IR spectral data for the respective lignins are shown in Figure 11.

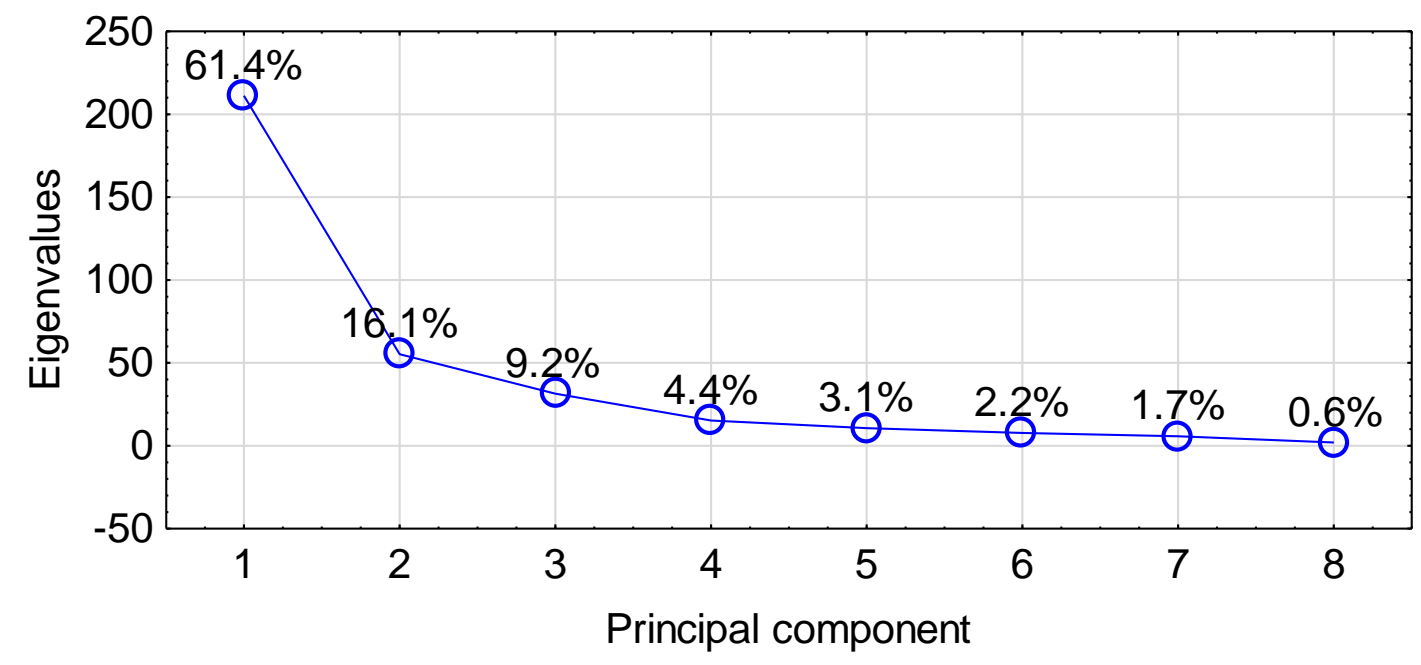

Figure 10. Scree plot of the eigenvalues of the principal components and their percentage contributions to describing the variability in the mid-IR data. 
The S-S lignin had a different structure to the rest of the lignin in the fingerprint region. The separation of the S-S sample could be attributed to the higher content of methoxyl groups and more S-units present as at 1420 $\mathrm{cm}^{-1}$, as opposed to S-A and S-A-P. L-S-D differed from L-S due to the reduction in lower molecular weight $\mathrm{H}$ and G-units as well as some ash and sulphur that have been removed during dialysis (as shown in Table 8 and Table 9). The S-A-P and L-S showed shoulders for conjugated ketones at $1650 \mathrm{~cm}^{-1}$, and they had fewer S-units compared to the other samples, which attributed to them forming a separate cluster in PC4. The separation of the P-S-A-P sample from the other samples could be attributed to its lower peak at $1211 \mathrm{~cm}^{-1}$ characteristic of stretching of phenolic $\mathrm{C}-\mathrm{OH}$ bonds and phenolic $\mathrm{C}-\mathrm{O}$ aromatic bonds. The P-S-A-P sample also had no peak at $1160 \mathrm{~cm}^{-1}$ attributed to ester bonds, which indicated that these bonds were cleaved during the phenolation reaction. Therefore, phenolation did lead to positive changes in the structure of the S-A-P sample as expected, leading to a higher reactivity for LPF synthesis (as discussed in section 4.2.3). The PCA groupings were thus governed more by the structure of the lignins, while no significant groupings were observed based on biomass origin, pulping method, isolation/purification method.

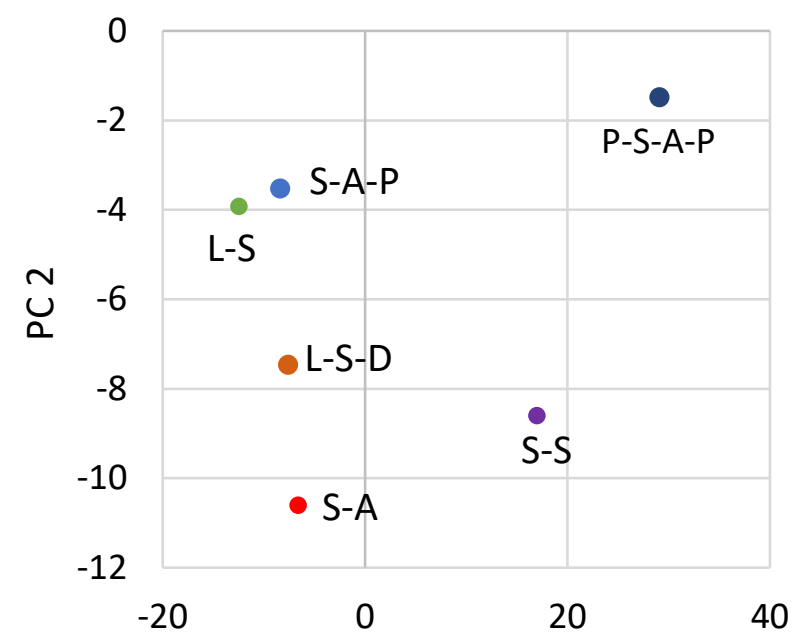

PC 1

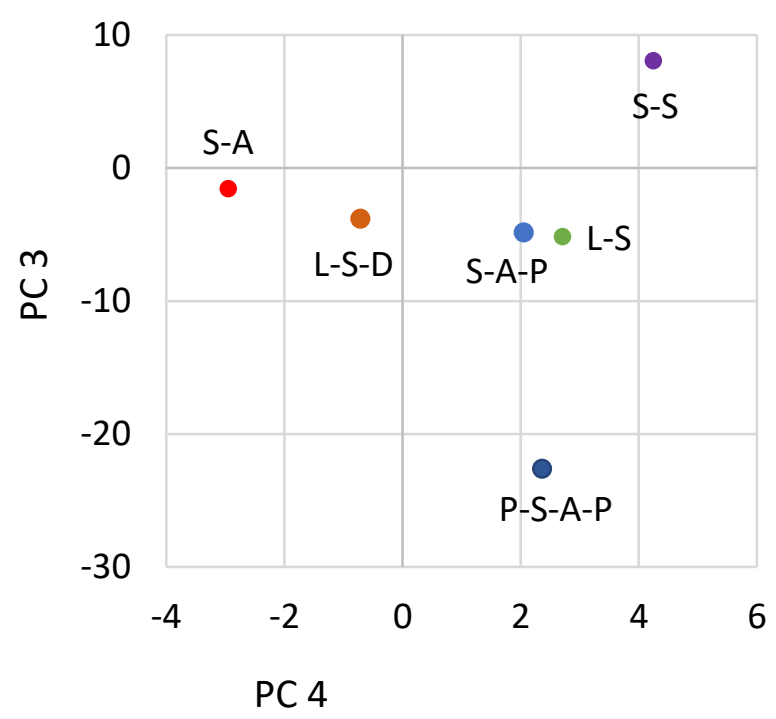

PC 4

Figure 11. PCA plots obtained from the mid-IR spectra of the lignin and lignosulphonate samples.

\subsubsection{TGA results of the lignin and lignosulphonate samples}

S-A-P was the most thermally stable followed by S-A, L-S, then S-S, with their maximum thermal degradation temperatures at $425^{\circ} \mathrm{C}, 375^{\circ} \mathrm{C}, 283{ }^{\circ} \mathrm{C}$, and $240{ }^{\circ} \mathrm{C}$, respectively. The curves for mass loss (TGA) and rate of mass loss (DTG) with temperature are shown in Figure 12. The differences observed in the thermal stability of the different lignins were attributed to the differences in their structures, which is known to correlate with the rigidity, molar mass distribution and degree of cross-linking of the polymeric chains (Khan \& Ashraf, 2007). The high thermal stability of S-A-P could be attributed to the higher content of G-units, which can contain condensed C-C bonds between the moieties at 5-5, that are more thermally stable. (Naron et al., 2017) Furthermore, the high ash content in some of the samples (L-S and S-S) could have caused a catalytic effect which could have led to degradation at lower temperatures (Naron et al., 2017). Lignin decomposition has been reported to occur from 200 to $500^{\circ} \mathrm{C}$ (Nsaful et al., 2015) and degradation start at temperatures between 150 and $275{ }^{\circ} \mathrm{C}$ (Collard \& Blin, 2014), so these corresponded to previous reports and was as expected. LPF 
resins are cured around $165{ }^{\circ} \mathrm{C}$, therefore the high thermal stability of these lignins would be favourable, as they would not degrade during the curing reaction.

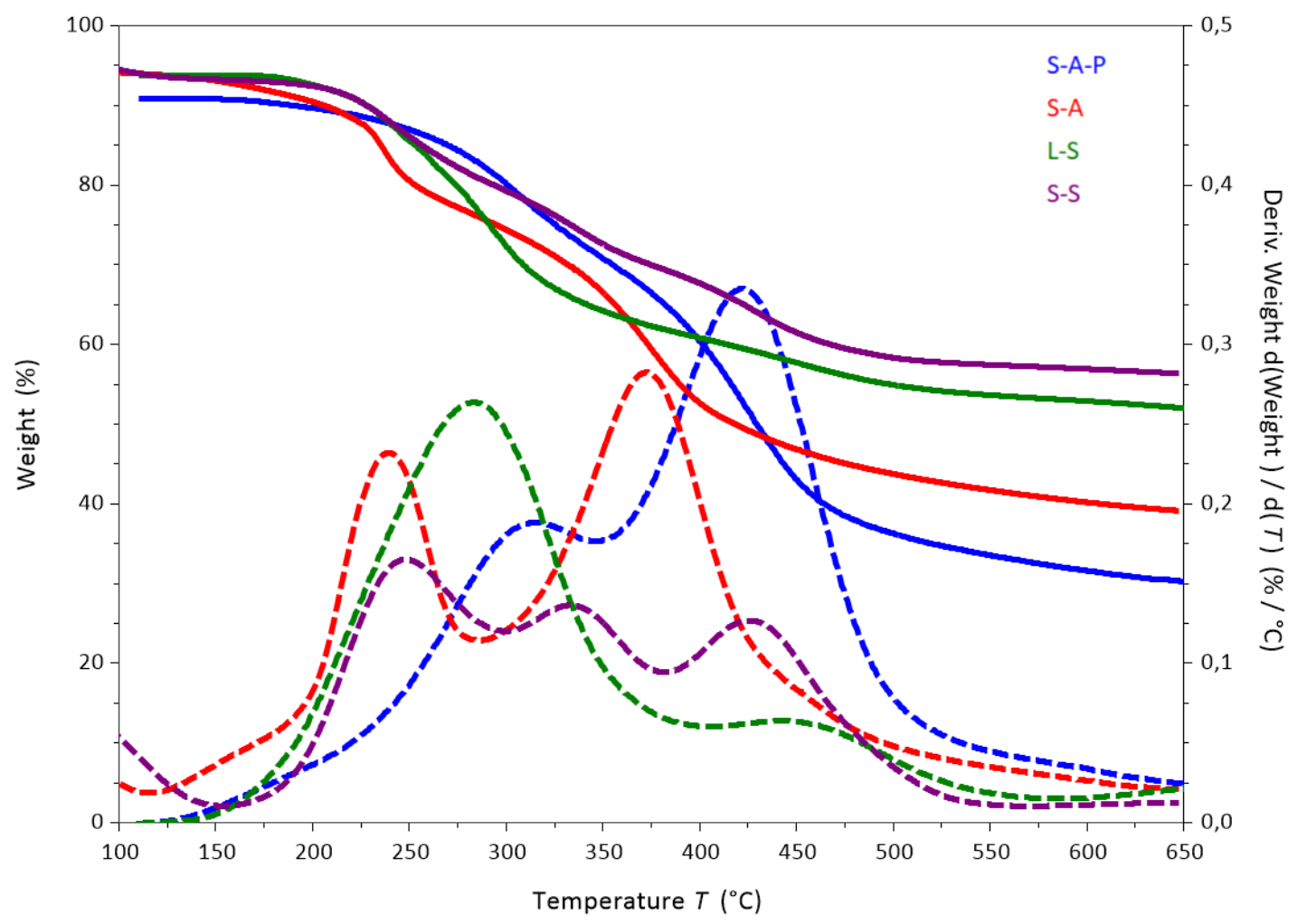

Figure 12. TGA and DTG graphs of the soda lignin and lignosulphonate samples.

The moisture in all of the samples was removed at $100{ }^{\circ} \mathrm{C}$ and all the samples retained more than $90 \%$ of their weight. From 150 to $275{ }^{\circ} \mathrm{C}$ dehydration of hydroxyl groups occurred. The lignins are thermally stable up to $200{ }^{\circ} \mathrm{C}$, after which they start to degrade. Weight loss in the region from 200 to $250{ }^{\circ} \mathrm{C}$ was from cleavage of the weak $\alpha$ - and $\beta$-aryl-alkyl-ether bonds between lignin units (Laurichesse \& Avérous, 2014). The H-units in grasses are mostly connected by $\beta-0-4$ bonds (Chen, 2014), which are easily degradable and could have been cleaved, thereby also removing carbohydrates during acid purification of S-A-P (Hidayati et al., 2020; Zhang et al., 2020), while S-A and S-S have a prominent peak in this region. Cleavage of aliphatic side chains from the aromatic ring occurred above $300{ }^{\circ} \mathrm{C}$, followed by cleavage of more stable inter-unit C-C bonds (5-5' phenyl bonds and 4-0-5' ether bonds) from 370 to $450^{\circ} \mathrm{C}$. Methoxyl groups are cleaved above $380{ }^{\circ} \mathrm{C}$, whereafter methylene bonds are cleaved. The peak in S-A-P above $400{ }^{\circ} \mathrm{C}$ could indicate that $5-5$ bonds are still present between the lignin units, which especially form between the G-units (Naron et al., 2017). At temperatures above $450{ }^{\circ} \mathrm{C}$ most bonds have been cleaved (Laurichesse \& Avérous, 2014). At high temperatures gaseous compounds (such as carbon, hydrogen, sulphur, nitrogen, oxygen, etc.) are formed and the residual ash consists mostly of mineral elements in oxide form (Chen, 2014). The higher weight residue of the S-S and L-S was attributed to the higher ash contents, which agreed with the ash content results. Maximum ash formation for soda lignin occurred from 550 to $580^{\circ} \mathrm{C}$, while for lignosulphonates the high ash formation required a temperature above $800{ }^{\circ} \mathrm{C}$. The high weight residue of the S-S lignin could also be attributed to the high content of ash present in the sample. The results were as expected and agreed with other reports (Marques 
et al., 2009; Naron et al., 2017). Both the low ash content and the indication of stable branched structures already formed in the S-A-P sample foreshadow good use of it for LPF applications.

\subsubsection{Screening of shear strength results of LPF resins made from soda lignin and lignosulphonate}

The lignin characterisations indicated that S-A-P was the most promising phenol replacement: From the structural compositional analysis, it had the lowest ash and sulphur content, and a high phenolic hydroxyl content. The mid-IR spectra confirmed a high hydroxyl content in S-A-P and showed the presence of H-, G-, and S-units. From the TGA results, S-A-P was also the most thermally stable which was attributed to its low ash content, and the higher content of G-units, which have condensed C-C bonds between the units that have a very high thermal stability (Naron et al., 2017).

Screening experiments were done where S-A-P, L-S, and L-S-D lignins were used to synthesise LPF100_(0,0) resins using the $C C D$ centre point experimental conditions. The results are shown in Figure 13. The $\mathrm{pH}$ value of the LPF resins should be in the range of $\mathrm{pH} 8$ to 13 to avoid lignin precipitation and maintain a high water resistance (Tonge, 2007). The $\mathrm{pH}$ values obtained for S-A-P, L-S, and L-S-D were in this range at $\mathrm{pH}$ values of $10.6,9.32$ and 8.9 , respectively.

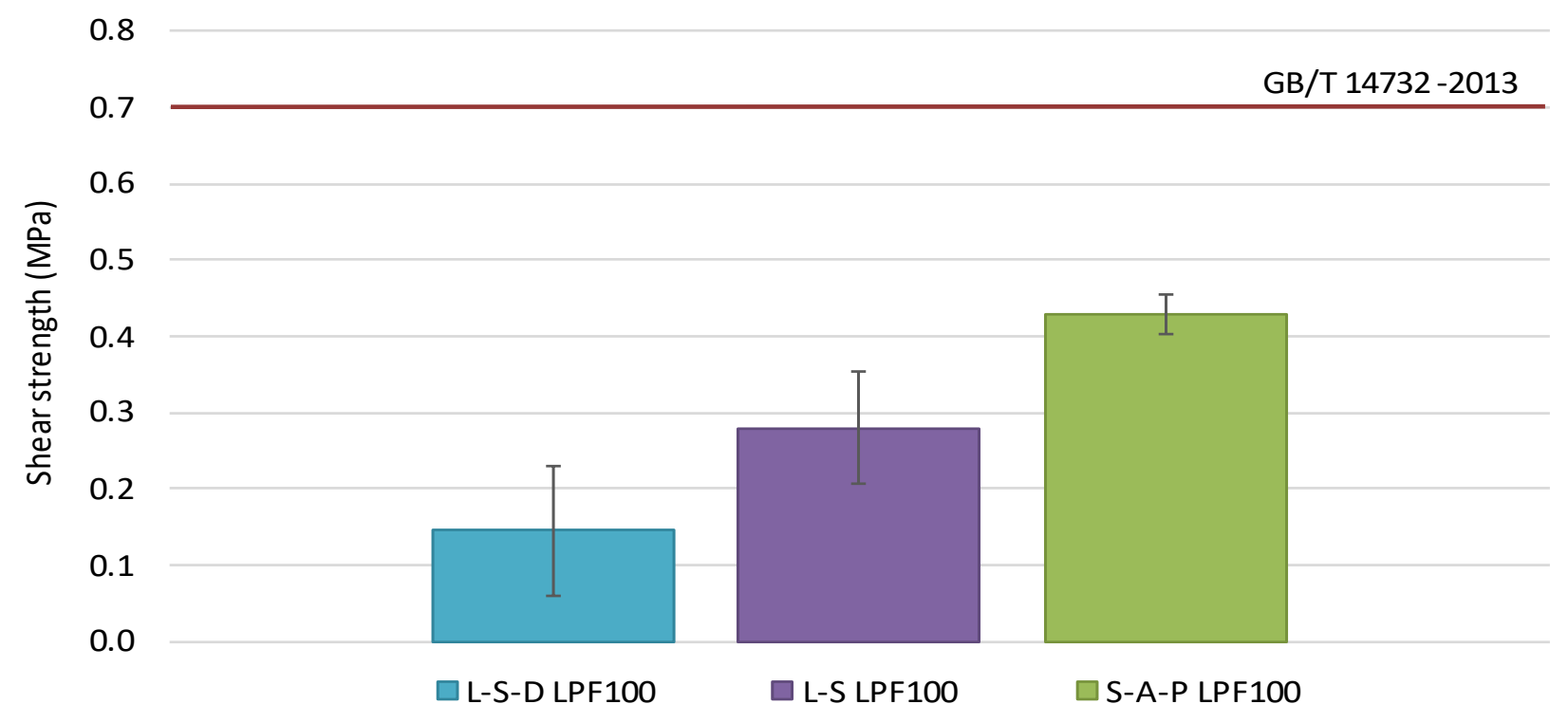

Figure 13. Comparison between the shear strengths of the lignosulphonate and soda lignin LPF100 resins made at centre point conditions.

These screening experiments verified the prediction from the lignin characterisations that S-A-P is a more promising phenol replacement for LPF resin applications. The shear strengths were determined to be $0.430 \pm$ $0.026 \mathrm{MPa}$ with S-A-P, $0.280 \pm 0.075 \mathrm{MPa}$ with L-S, and $0.145 \pm 0.086 \mathrm{MPa}$ with L-S-D. The lignosulphonate samples did not come near the standard requirement of $0.70 \mathrm{MPa}$, which was expected due to its high ash content and lower reactivity, and corresponded with previous reports (Alonso et al., 2004, 2005; Ghorbani, Konnerth, et al., 2018). The lower performance of L-S-D compared to L-S was attributed to its lower phenolic hydroxyl content and dialysis removing the low molecular weight units, thereby lowering the reactivity of L-SD. Thus, further optimisations and characterisations were done using the S-A-P lignin. 
Lignosulphonates proved in previous reports to be viable as replacements of phenol either at low substitution levels (Alonso et al., 2001; Ghorbani, Konnerth, et al., 2018) or by adding additives during the adhesive formulation. Kalami et al. (2018) used different lignins and produced LPF100 resins which were able to produce shear strengths above $0.70 \mathrm{MPa}$ as required by the standard. One of the resins was made using a lignosulphonate from softwood and produced plywood with a shear strength of $1.8 \mathrm{MPa}$, but the free formaldehyde content did not meet industrial standard requirements. Furthermore, the resin was dissolved in $\mathrm{NaOH}$, and wheat flour and alder bark (modal) was added to formulate the adhesive, which contributed to the good performance. Govender et al. (2020) proved that cross-linkers and hardeners are successful in enhancing resin properties and could also be applied to enable LPF100 resins to adhere to standard regulations.

\subsection{CCD shear strength results}

Different ranges of the phenolation temperature, $\mathrm{NaOH} / \mathrm{PL}$ molar ratio and F/PL molar ratio were investigated to maximise the shear strength attainable at $60 \%, 80 \%$ and $100 \%$ substitution. The substitution level indicates the total weight percentage lignin incorporated in the phenolation and synthesis, with the residual being the total phenol incorporated (with no more lignin or phenol added later). This lignin and phenol were first reacted in alkaline conditions, whereafter formaldehyde was added to this lignin-phenol solution to start the addition part of the synthesis reaction. The different LPF resins were labelled where the number directly after 'LPF' indicated the substitution percentage, followed by brackets which indicated the level of each parameter used in the order: $\mathrm{NaOH} / \mathrm{PL}$ molar ratio, F/PL molar ratio, and then the phenolation temperature. In the case of LPF100 resins which only had the first two parameters optimised in its CCD, the third was omitted.

$$
\text { LPF [substitution \%]_([NaOH/PL], [F/PL], [Temperature]) }
$$

\subsubsection{Statistical analysis of the CCD data}

A regression model was built, and an ANOVA was performed for each CCD to determine if the parameters that were varied during the phenolation and LPF synthesis reaction had a significant effect on the mechanical performance of the LPF resins, and to determine at what levels of the parameters the shear strength would be at a maximum. The ANOVA also included the combined linear effects of parameters on the shear strength (for example the combined linear effects of parameters 1 and 2 would be indicated as $1 \mathrm{~L}$ by $2 \mathrm{~L}$ ). Statistical significance was verified using the F-test (significant if $F>F_{\text {critical }}$ ) and the $p$-value test (significant if $p<\alpha$ ). An $\alpha$ value of 0.10 was assumed to be significant as this improved the model. Chen, et al., (2019) also used an alpha of 0.1 to improve the regression model they used. Furthermore, the process had many other factors that were not optimised (such as reaction times and curing parameters) that could influence the results in the statistical analysis.

\subsubsection{LPF100 resins}

The regression from the ANOVA as reported in Table 11, had a low coefficient of multiple determination $\left(R^{2}\right)$ of $31 \%$, which was attributed to the small number of data points included in the experimental design of the LPF100 resins with 12 runs, as compared to the LPF60 and LPF80 resins which had 18 runs, as the phenolation temperature was also varied. There were also no significant parameters with $p<0.1$ and a significant lack of 
fit was also found $\left(F<F_{\text {critical }}\right)$. This should mean that the null hypothesis cannot be rejected, therefore none of the parameters would influence the shear strength. The regression model was still used to predict operating conditions that would maximise the shear strength, as the results were verified experimentally.

Table 11. ANOVA results of the CCD shear strengths optimisation of the LPF100 resins.

\begin{tabular}{lccccc}
\hline & SS & df & MS & F & p \\
\hline (1) NaOH/PL molar ratio(L) & 0.0397 & 1 & 0.040 & 0.79 & 0.41 \\
NaOH/PL molar ratio (Q) & 0.0591 & 1 & 0.059 & 1.18 & 0.32 \\
(2) F/PL molar ratio(L) & 0.0204 & 1 & 0.020 & 0.41 & 0.55 \\
F/PL molar ratio (Q) & 0.0231 & 1 & 0.023 & 0.46 & 0.52 \\
1L by 2L & 0.0112 & 1 & 0.011 & 0.22 & 0.65 \\
Error & 0.3007 & 6 & 0.050 & & \\
\hline Lack of Fit & 0.2981 & 3 & 0.099 & 112.37 & 0.00 \\
Pure Error & 0.0027 & 3 & 0.001 & & \\
Total SS & 0.4369 & 11 & & & \\
\hline
\end{tabular}

Figure 14 shows the desirability profile obtained for the LPF100 resins to maximise the shear strength. The highest shear strength according to the model was $0.663 \mathrm{MPa}$; however, $0.786 \mathrm{MPa}$ was reached with experiments. From the desirability profiling, increasing the $\mathrm{NaOH} / \mathrm{PL}$ ratio increased the shear strength sharply with its optimum at 0.477 , whereafter the shear strength decreased slightly again. The shear strength was at its highest with the F/PL ratio at 3; however, it did not seem to have a sharp effect on the shear strength. These agreed with some previous reports that also indicated high $\mathrm{NaOH}$ and $\mathrm{F}$ requirements for $\mathrm{LPF}$ synthesis (Khan et al., 2004; Abdelwahab \& Nassar, 2011; Ghorbani et al., 2016), and the high requirements for this study were attributed to the higher incorporation of lignin with lower reactivity, compared to other reports which had lower requirements at low substitution levels.
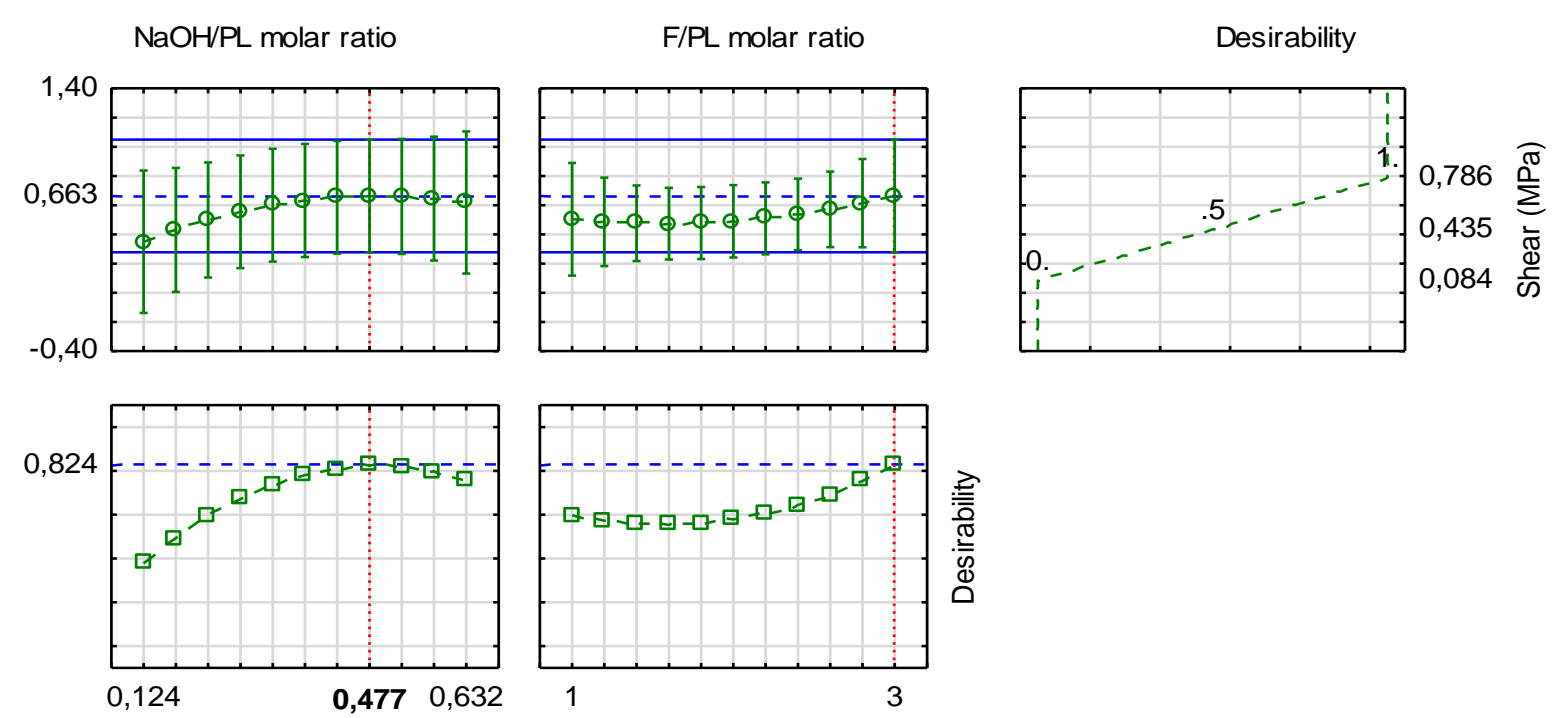

Figure 14. Profiles for predicted values and desirability of the CCD shear strength results of the LPF100 resins. The green line represents the predicted curve, the red lines represent optimums and the blue lines the upper and lower bounds. 


\subsubsection{LPF80 resins}

The ANOVA performed on the CCD at $80 \%$ substitution reported an $\mathrm{R}^{2}$ that described $80.6 \%$ of the variability in the shear strength, as shown in Table 12. The $p$-value of 0.04 was $<0.1$, and $F=6.84>F_{\text {critical }}=5$, which indicated statistical significance. The null hypothesis could therefore be rejected, as some of the parameters did have a statistically significant effect on the shear strength. Four of the parameters were found to be significant, which included the quadratic term of the $\mathrm{NaOH} / \mathrm{PL}$ ratio, and three linear terms namely the phenolation temperature, F/PL ratio, and the combined effect of the $\mathrm{NaOH} / \mathrm{PL}$ ratio and temperature.

Table 12. ANOVA results of the CCD shear strengths optimisation of the LPF80 resins.

\begin{tabular}{lccccc}
\hline & $\mathrm{SS}$ & $\mathrm{df}$ & $\mathrm{MS}$ & $\mathrm{F}$ & $\mathrm{p}$ \\
\hline (1) NaOH/PL molar ratio(L) & 0.019 & 1 & 0.019 & 0.81 & 0.39 \\
NaOH/PL molar ratio (Q) & $\mathbf{0 . 1 0 6}$ & $\mathbf{1}$ & $\mathbf{0 . 1 0 6}$ & $\mathbf{4 . 4 0}$ & $\mathbf{0 . 0 7}$ \\
(2) Temperature $\left({ }^{\circ} \mathrm{C}\right)(\mathrm{L})$ & $\mathbf{0 . 4 3 8}$ & $\mathbf{1}$ & $\mathbf{0 . 4 3 8}$ & $\mathbf{1 8 . 2 1}$ & $<\mathbf{0 . 0 1}$ \\
Temperature $\left({ }^{\circ} \mathrm{C}\right)(\mathrm{Q})$ & 0.004 & 1 & 0.004 & 0.16 & 0.70 \\
(3) F/PL molar ratio (L) & $\mathbf{0 . 1 1 6}$ & $\mathbf{1}$ & $\mathbf{0 . 1 1 6}$ & $\mathbf{4 . 8 4}$ & $\mathbf{0 . 0 6}$ \\
$\mathrm{F} / \mathrm{PL}$ molar ratio (Q) & 0.002 & 1 & 0.002 & 0.06 & 0.81 \\
$\mathbf{1 L}$ by 2L & $\mathbf{0 . 1 0 1}$ & $\mathbf{1}$ & $\mathbf{0 . 1 0 1}$ & $\mathbf{4 . 2 2}$ & $\mathbf{0 . 0 7}$ \\
1L by 3L & 0.011 & 1 & 0.011 & 0.48 & 0.51 \\
2L by 3L & 0.040 & 1 & 0.040 & 1.67 & 0.23 \\
Error & 0.216 & 9 & 0.024 & & \\
\hline Lack of Fit & 0.194 & 5 & 0.039 & 6.84 & 0.04 \\
Pure Error & 0.023 & 4 & 0.006 & & \\
Total SS & 1.115 & 18 & & & \\
\hline
\end{tabular}

The desirability profiling was shown in Figure 15. The highest shear strength according to the regression model was $1.04 \mathrm{MPa}$, which correlated well with the highest shear strength of $1.09 \mathrm{MPa}$ obtained during experimental runs at $80 \%$ substitution. The shear strength decreased gradually with an increase in $\mathrm{NaOH} / \mathrm{PL}$ molar ratio up to 0.554 until a sharp increase was observed with the best $\mathrm{NaOH} / \mathrm{PL}$ molar ratio at 0.632 . The F/PL did not seem to have a big impact on the shear strength when looking at its plot which was close to a horizontal line. The $\mathrm{NaOH} / \mathrm{PL}$ required was higher than expected, while the F/PL ratio was as expected from previous reports, as compared in Addendum A. The shear strength was greatly affected by the phenolation reaction temperature with the highest shear strength reached at a temperature of $80^{\circ} \mathrm{C}$. This phenolation temperature was low compared to previous reports (Yang et al., 2014; Gan \& Pan, 2019). These optimum conditions corresponded to that found in the LPF80 experimental runs as reported in Addendum C. 

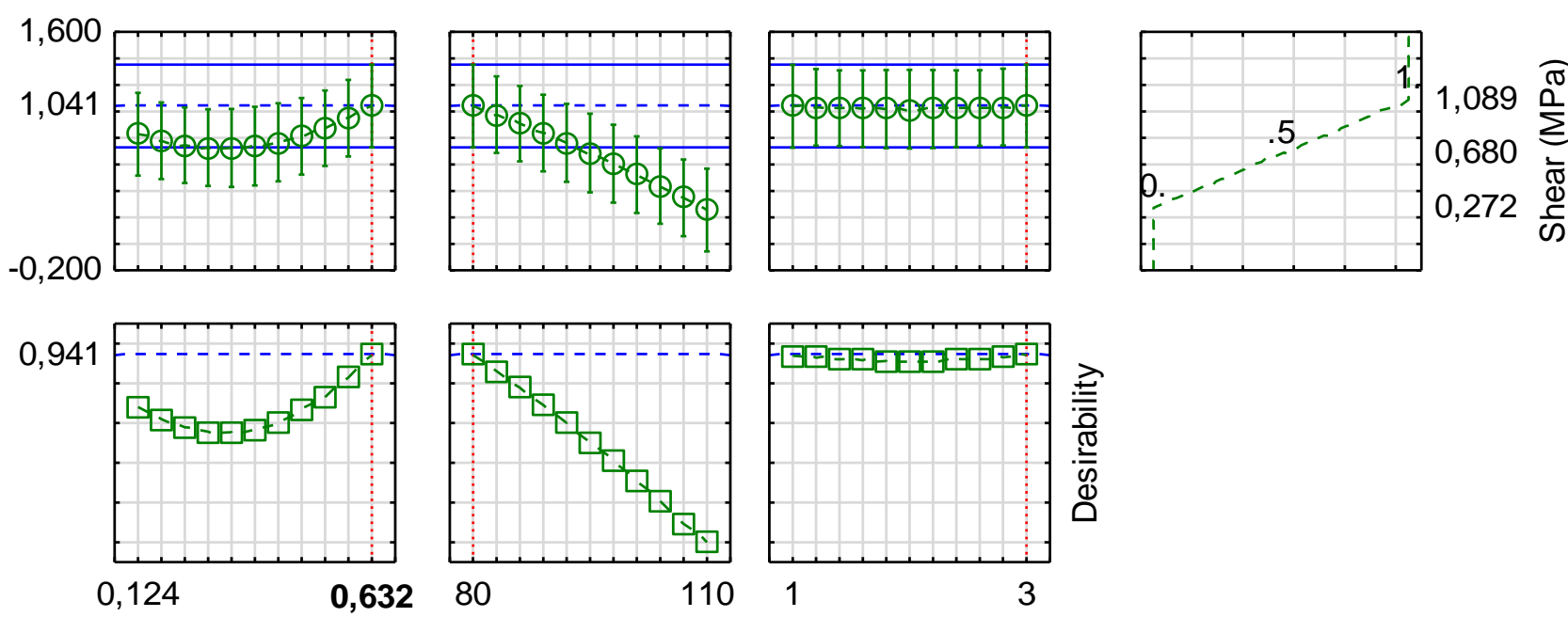

Figure 15. Profiles for predicted values and desirability of the CCD shear strength results of the LPF80 resins. The green line represents the predicted curve, the red lines represent optimums and the blue lines the upper and lower bounds.

\subsubsection{LPF60 resins}

The regression equation with an $\mathrm{R}^{2}$ of $70.7 \%$ described the variability in the shear strength of the LPF60 resins, as reported in

Table 13. Two of the terms had a significant effect on the shear strength: the linear terms of the phenolation reaction temperature and the F/PL molar ratio. The $p$-value was $<0.1$, and $F=25>F_{\text {critical }}=5$, which indicated that the model was statistically significant and that the null hypothesis could be rejected, as some of the parameters had a statistically significant effect on the shear strength.

Table 13. ANOVA results of the CCD shear strength optimisation of the LPF60 resins.

\begin{tabular}{lccccc}
\hline & $\mathrm{SS}$ & $\mathrm{df}$ & $\mathrm{MS}$ & $\mathrm{F}$ & $\mathrm{p}$ \\
\hline (1) NaOH/PL molar ratio(L) & 0.032 & 1 & 0.032 & 1.00 & 0.35 \\
$\mathrm{NaOH} / \mathrm{PL}$ molar ratio (Q) & 0.033 & 1 & 0.033 & 1.02 & 0.34 \\
$(\mathbf{2})$ Temperature $\left({ }^{\circ} \mathrm{C}\right)(\mathrm{L})$ & $\mathbf{0 . 1 1 6}$ & $\mathbf{1}$ & $\mathbf{0 . 1 1 6}$ & $\mathbf{3 . 5 8}$ & $\mathbf{0 . 1 0}$ \\
Temperature $\left({ }^{\circ} \mathrm{C}\right)(\mathrm{Q})$ & 0.004 & 1 & 0.004 & 0.13 & 0.72 \\
(3) F/PL molar ratio (L) & $\mathbf{0 . 3 6 1}$ & $\mathbf{1}$ & $\mathbf{0 . 3 6 1}$ & $\mathbf{1 1 . 2 0}$ & $\mathbf{0 . 0 1}$ \\
$\mathrm{F} / \mathrm{PL} \mathrm{molar} \mathrm{ratio} \mathrm{(Q)}$ & 0.005 & 1 & 0.005 & 0.17 & 0.69 \\
1L by 2L & 0.029 & 1 & 0.029 & 0.90 & 0.37 \\
1L by 3L & 0.001 & 1 & 0.001 & 0.04 & 0.84 \\
2L by 3L & 0.018 & 1 & 0.018 & 0.56 & 0.47 \\
Error & 0.258 & 8 & 0.032 & & \\
\hline Lack of Fit & 0.252 & 5 & 0.050 & 24.99 & 0.01 \\
Pure Error & 0.006 & 3 & 0.002 & & \\
Total SS & 0.880 & 17 & & & \\
\hline
\end{tabular}


The highest shear strength from the regression was $1.02 \mathrm{MPa}$, which correlated with the maximum of 0.987 MPa obtained from the experimental runs at $60 \%$ substitution. The desirability profile in Figure 16 indicated that at a low $\mathrm{NaOH} / \mathrm{PL}$ molar ratio of 0.124 the shear strength was the highest, and it decrease as the $\mathrm{NaOH}$ concentration increased until it remained relatively constant at a $\mathrm{NaOH} / \mathrm{PL}$ molar ratio of above 0.36 . The phenolation reaction temperature and the F/PL molar ratios seemed to have an almost linear effect on the shear strength, where the optimum temperature was at the higher bound at $110^{\circ} \mathrm{C}$, while the optimum $\mathrm{F} / \mathrm{PL}$ molar ratio was at the lower bound at 1 . The phenolation temperature correlated with previous reports ( $\mathrm{Hu}$ et al., 2012; Ghorbani, Konnerth, et al., 2018), while the $\mathrm{NaOH} / \mathrm{PL}$ and F/PL ratios were lower than expected, as compared to previous reports (Sarkar \& Adhikari, 2000; Ghorbani et al., 2016) in Addendum A. These optimum conditions corresponded to the second-highest shear strength at $60 \%$ substitution found in the experimental runs, as reported in Addendum B.

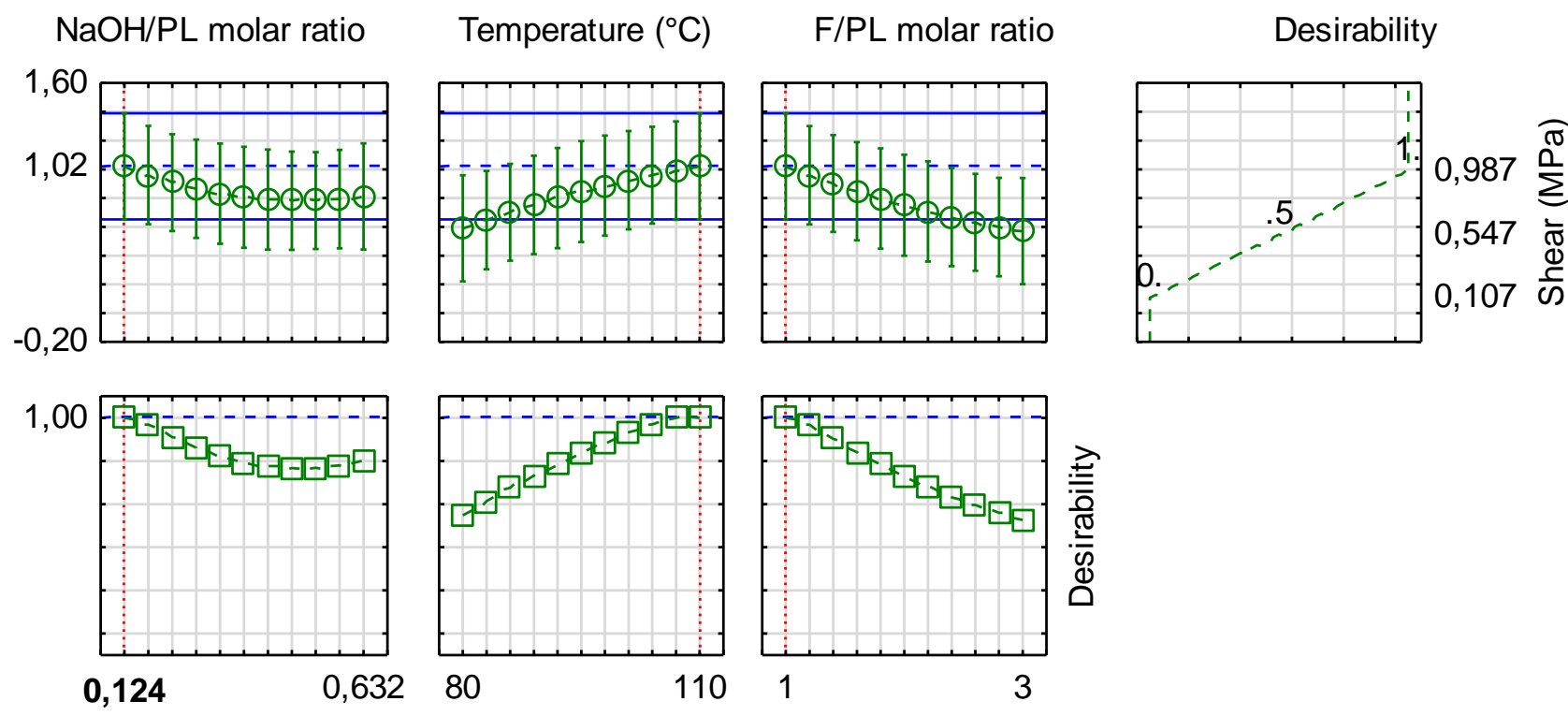

Figure 16. Profiles for predicted values and desirability of the CCD shear strength results of the LPF60 resins. The green line represents the predicted curve, the red lines represent optimums and the blue lines the upper and lower bounds.

\subsubsection{The effect of phenolation modification on the lignin characteristics}

The phenolic hydroxyl content of S-A-P increased from $1.70 \mathrm{mmol} / \mathrm{g}$ sample by $105 \%$ (up to $3.48 \mathrm{mmol} / \mathrm{g}$ ) and $124 \%$ (up to $3.81 \mathrm{mmol} / \mathrm{g}$ ) after phenolation at $60 \%$ and $80 \%$ substitution, respectively. Table 14 shows the phenolic hydroxyl contents obtained after phenolation of S-A-P at different reaction conditions. The higher phenolic hydroxyl content obtained at $60 \%$ was expected, as more phenol was added at $60 \%$ substitution, compared to at $80 \%$ substitution, where more lignin was present which had less phenolic hydroxyl groups compared to phenol.

The highest phenolic hydroxyl contents were observed at a $\mathrm{NaOH} / \mathrm{PL}$ molar ratio of 0.378 for both substitution levels and phenolation temperatures of 110 and $80{ }^{\circ} \mathrm{C}$ at $60 \%$ and $80 \%$ substitution, respectively. The lower temperature required at $80 \%$ substitution could be from the higher incorporation of lignin with more branched 
units compared to phenol, which could have promoted bulk contact (Siddiqui et al., 2017), leading to faster initiation of the phenolation reaction. Furthermore, condensation of the phenolic hydroxyls could have occurred at too high temperatures and too long reaction times, which could then lead to a decrease in phenolic hydroxyl content (El Mansouri, Farriol \& Salvadó, 2006; Zhao et al., 2016).

Table 14. Phenolic hydroxyl content of the phenolated lignin samples at $80 \%$ and $60 \%$ substitution, at different $\mathrm{NaOH} / \mathrm{PL}$ molar ratios and phenolation temperatures.

\begin{tabular}{ccccc}
\hline \multirow{2}{*}{$\begin{array}{c}\text { NaOH/PL } \\
\text { molar ratio }\end{array}$} & $\begin{array}{c}\text { Temperature } \\
\left({ }^{\circ} \mathrm{C}\right)\end{array}$ & \multicolumn{2}{c}{$\begin{array}{c}\text { Phenolic hydroxyl content } \\
\text { (mmol/g sample) }\end{array}$} \\
\cline { 3 - 5 } & & $80 \%$ & $60 \%$ \\
\hline 0.124 & 80 & 2.01 & 3.08 \\
0.124 & 110 & 3.31 & 2.91 \\
0.632 & 80 & 2.52 & 3.63 \\
& 0.632 & 110 & 2.85 & 3.16 \\
& 0.124 & 95 & 2.58 & 3.55 \\
& 0.632 & 95 & 3.05 & 3.02 \\
& 0.378 & 80 & 3.48 & 3.62 \\
& 0.378 & 110 & 3.16 & 3.81 \\
\hline Centre points: & 0.378 & 95 & $2.89 \pm 0.182$ & $3.62 \pm 0.636$ \\
\hline
\end{tabular}

Figure 17 confirmed structural changes as was expected after phenolation of S-A-P (at $68 \%$ substitution under optimal conditions as discussed in section 4.2.5). A low wavelength shift occurred from 3366 to $3335 \mathrm{~cm}^{-1}$ after phenolation indicating more hydrogen bonds present, which were attributed to more phenolic hydroxyl groups present from the phenol incorporated (Taleb et al., 2020). S-A-P had smaller bands at 2940 (C-H stretching) and $2880 \mathrm{~cm}^{-1}$ (aliphatic methyl and methylene groups) which agreed with previous reports (Ghorbani, Konnerth, et al., 2018). A new peak was observed at $2750 \mathrm{~cm}^{-1}$ from aromatic $\mathrm{C}-\mathrm{H}$ stretching from new aromatic rings in the phenolated lignin structure (Taleb et al., 2020). The shoulder band around $1265 \mathrm{~cm}^{-1}$ and the peak at $1160 \mathrm{~cm}^{-1}$ disappeared which indicated that ether bonds were cleaved after the phenolation reaction (Zhao et al., 2016; Hussin et al., 2017; Ghorbani, Konnerth, et al., 2018). The peak at $1215 \mathrm{~cm}^{-1}$ became more intensive indicating more $\mathrm{C}-\mathrm{O}$ stretching of phenolic hydroxyl groups (Ghorbani, Konnerth, et al., 2018). P-S-A-P had a new peak at $754 \mathrm{~cm}^{-1}$, attributed to the linkages formed between the ortho- or para-positions of phenol, and the $\alpha$-hydroxyl groups on the lignin side chain when phenol attached to the lignin aliphatic chain (Alonso et al., 2005). 


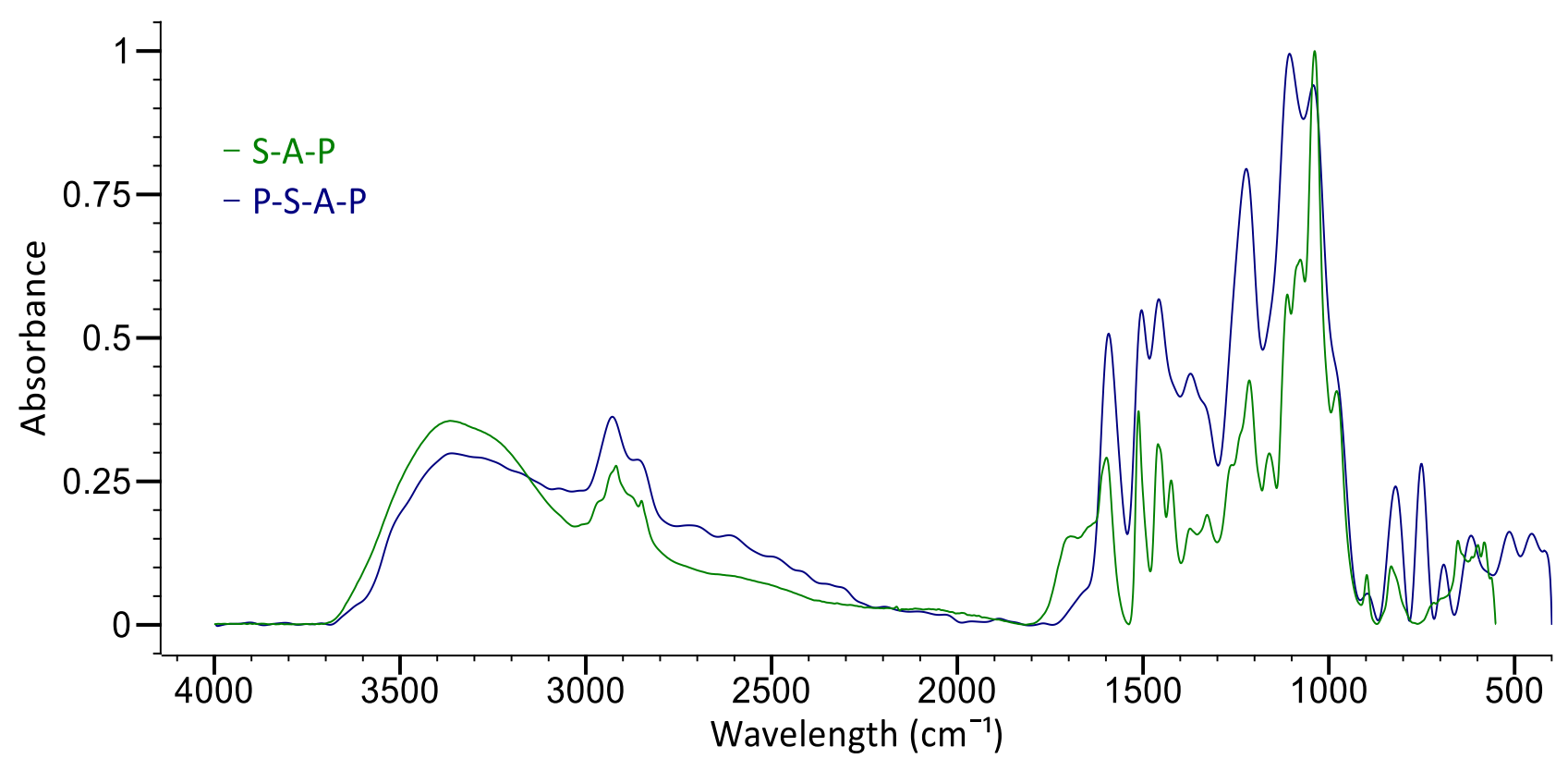

Figure 17. The mid-IR spectra of the purified soda lignin before and after phenolation.

\subsubsection{The effect of the phenolation reaction temperature on the shear strength}

The highest shear strength at $80 \%$ and $60 \%$ substitution was obtained at phenolation temperatures of $80{ }^{\circ} \mathrm{C}$ and $110^{\circ} \mathrm{C}$, respectively, as shown in Figure 18. The phenolation temperatures that gave the highest phenolic hydroxyl content (as discussed in 4.2.2) also gave the highest shear strength. This indicated that a high phenolic hydroxyl content was favourable in the LPF synthesis reaction, which then produced a high shear strength. When looking at the combined effect from $60 \%$ to $80 \%$ substitution, the highest shear strength was at $80{ }^{\circ} \mathrm{C}$.
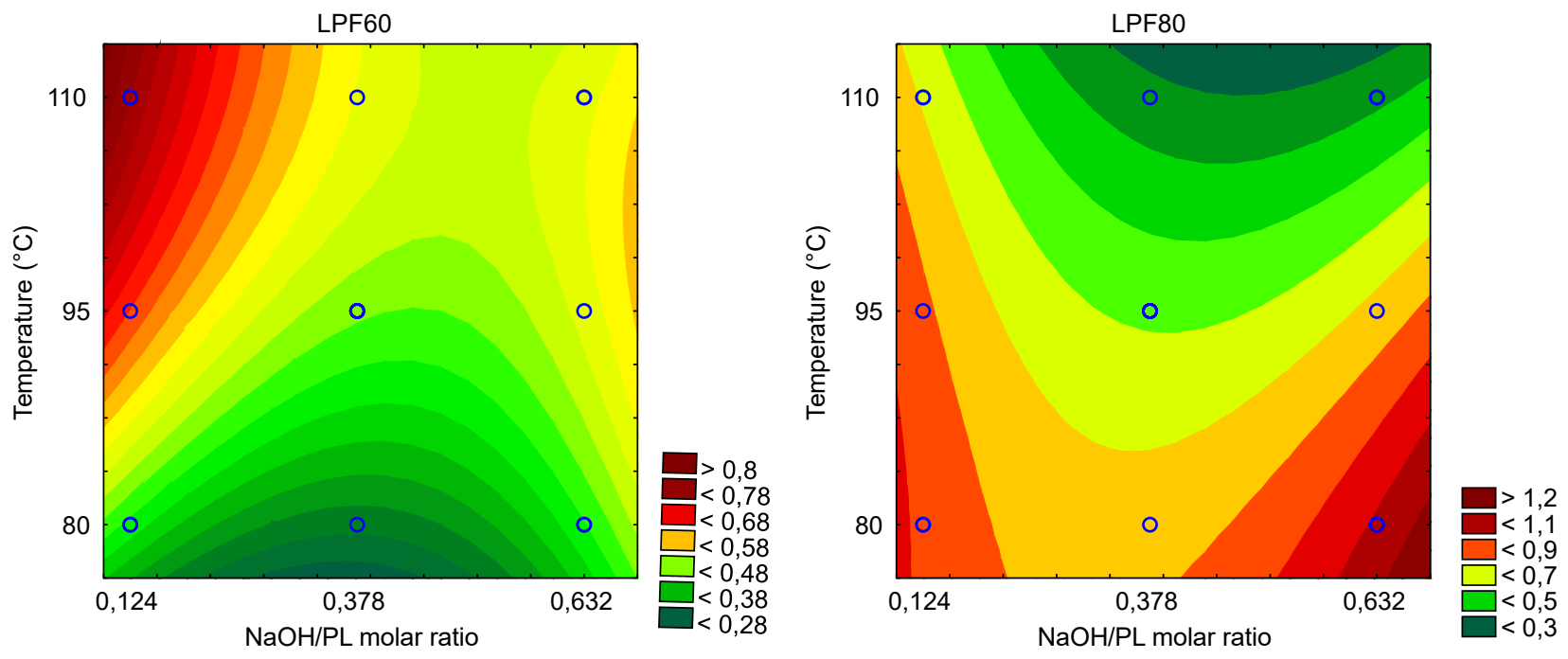


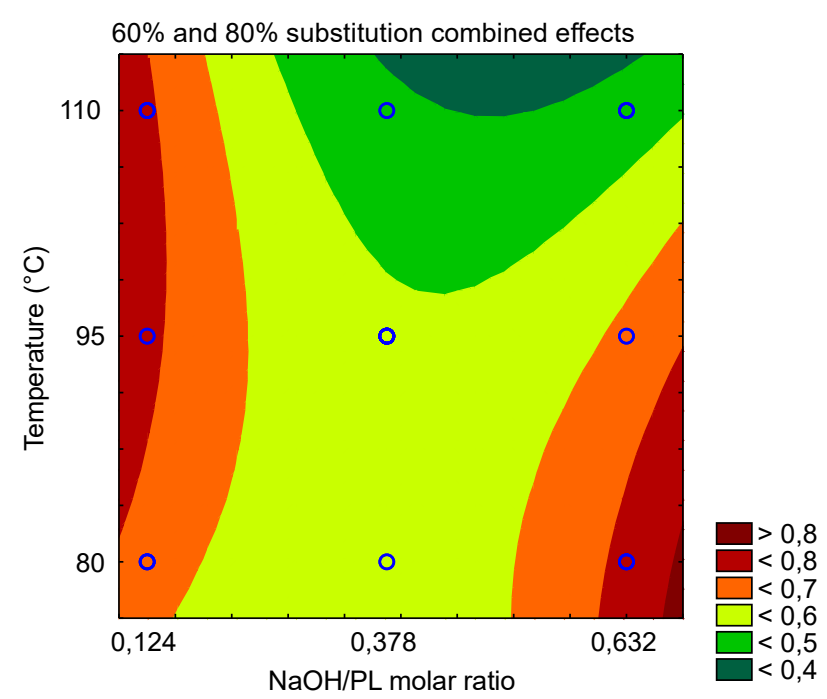

Figure 18. Contour plots of the effect of the $\mathrm{NaOH} / \mathrm{PL}$ ratio and phenolation reaction temperature on the shear strength (MPa).

\subsubsection{The effect of the NaOH/PL and F/PL molar ratios on the shear strength}

Phenolation of S-A-P at $60 \%$ and $80 \%$ substitution allowed high shear strengths to be reached at low sodium hydroxide concentrations and low formaldehyde additions required, which was attributed to phenolation enhancing the reactivity and hence lowering the activation energy required. Contour plots of the effects of $\mathrm{NaOH} / \mathrm{PL}$ and F/PL molar ratios on the shear strength are shown in Figure 19. The investigated molar ratio of $\mathrm{NaOH} / \mathrm{PL}$ ranged from 0.124 to 0.632 and the F/PL molar ratio ranged from one to three.

For the LPF60 and LPF80 resins and the combined response, the highest shear strength was obtained at the lower bound $\mathrm{NaOH} / \mathrm{PL}$ ratio of 0.124 . The lower $\mathrm{NaOH}$ addition desired at $60 \%$ and $80 \%$ could be explained by the higher reactivity of the phenolated lignin, which then required a lower activation energy and hence lower catalyst addition. Furthermore, an excess $1 \mathrm{M} \mathrm{NaOH}$ could cause a reduction in the adhesive strength (Abdelwahab \& Nassar, 2011; Zhao et al., 2016), and too high $\mathrm{NaOH}$ concentrations could lead to side reactions occurring. These could include the Cannizzaro reaction where the formaldehyde condenses with itself; therefore, less formaldehyde would be available to react to form large cross-linked structures (Sarkar \& Adhikari, 2000), or the Tollens reaction where the formaldehyde attaches to the lignin side chain carbonyl groups instead of the aromatic ring (Matsushita, 2015).

For the LPF100 resin which had a lower reactivity, more $\mathrm{NaOH}$ catalyst was required to overcome the higher activation energy, and an optimum was obtained at a $\mathrm{NaOH} / \mathrm{PL}$ molar ratio of 0.477 . An increase in catalyst concentration led to an acceleration of the condensation reaction involving methylol groups (Khan et al., 2004), yielding higher adhesive strengths. 

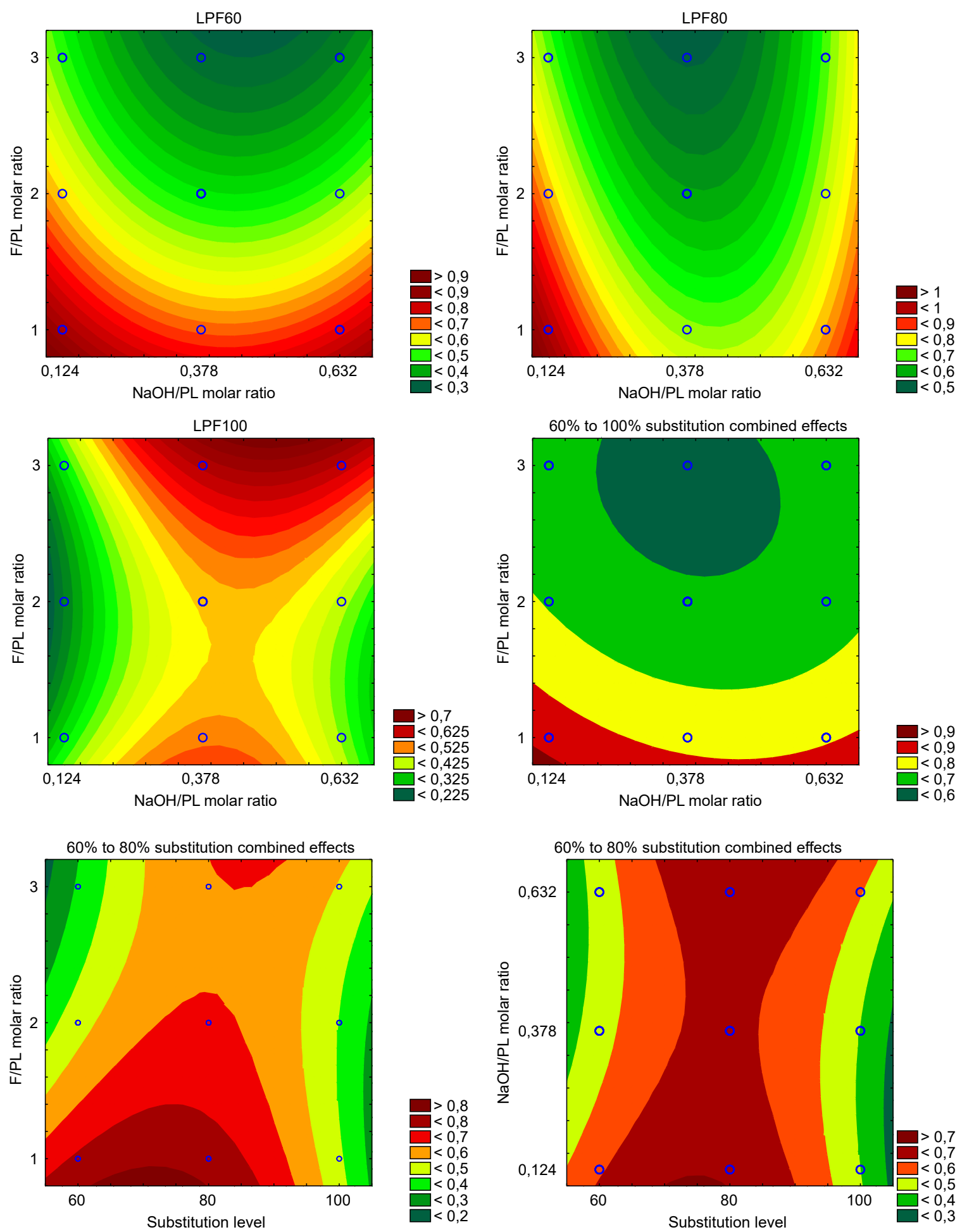

Figure 19. Contour plots of the effects of F/PL and $\mathrm{NaOH} / \mathrm{PL}$ molar ratios on the shear strength (MPa) at different substitution levels.

The highest shear strength was obtained at a F/PL ratio of one for the LPF60, LPF80, and the combined response, while a ratio of three was required for LPF100. Again the higher reactivity and reduced steric hindrance of the phenolated lignins would require a lower activation energy, and hence require less formaldehyde to promote the forward reaction (Siddiqui et al., 2017) as compared to LPF100. Previous reports support that formaldehyde addition should not be largely in excess to promote the forward reaction as excess 
formaldehyde could cause excessive hydrogen bond formation between molecules hindering cross-linking (Khan et al., 2004). It could also cause toxic emissions, and an excess of formalin solution ( $35 \%$ formaldehyde) could also reduce cross-linking and hence shear strength.

When comparing these molar ratio requirements to previous reports (Moubarik et al., 2013; Zhang et al., 2013; Ghorbani et al., 2016; Govender, Majeke, et al., 2020), which are summarised in Addendum A, phenolation allowed high shear strengths to be reached at lower formaldehyde and sodium hydroxide additions required, which was attributed to phenolation enhancing the reactivity and hence lowering the activation energy required. The optimum $\mathrm{NaOH} / \mathrm{PL}$ molar ratio of 0.477 for LPF100 to maximise the shear strength agreed with previous reports. The F/PL ratio desired was higher compared to previous reports and was attributed to the high percentage incorporation of the less reactive soda lignin, requiring a higher formaldehyde content to overcome the higher activation energy and promote the forward synthesis reaction. Previous reports used lower substitution levels (Ghorbani et al., 2016; El Mansouri et al., 2018), or used additives during adhesive formulation (Kalami et al., 2018; Govender, Majeke, et al., 2020). Commercial PF is synthesised at a NaOH/P molar ratio of 0.66 and a F/P molar ratio of 5.6 (Tachon, Benjelloun-Mlayah \& Delmas, 2016), so these LPF resins could be synthesised with a lower $\mathrm{NaOH}$ concentration and formaldehyde addition required. The effect of substitution level on the shear strength

The shear strengths of the S-A-P LPF100, LPF80 and LPF60 resins obtained from the CCD experiments conducted under different experimental conditions are shown in Figure 20. The whiskers represented the maximum and minimum values, with no outliers outside of these regions. The bottom line, middle line, and top line of the box represented the first quartile, median and third quartile, respectively. The ' $X$ ' represented the mean of the dataset.

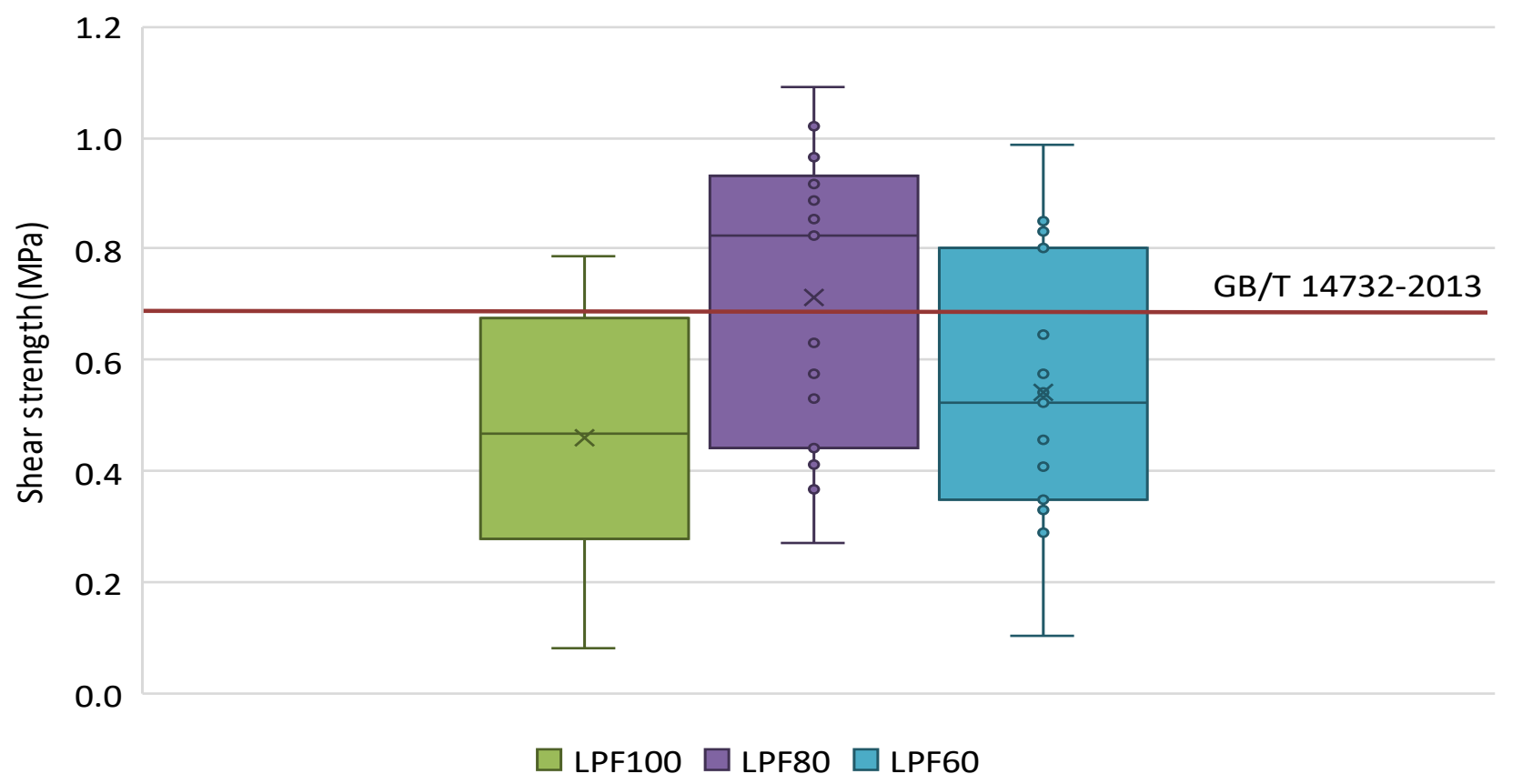

Figure 20. Shear strengths of the S-A-P LPF100, LPF80, and LPF60 resins made at different reaction conditions, as determined by the experimental CCDs. 
A shear strength above $0.70 \mathrm{MPa}$ as specified by the GB/T 1473-2013 standard were attainable at all substitution levels, even at LPF100 if favourable reaction conditions specific to that substitution level was used. The maximum shear strengths at $60 \%, 80 \%$ and $100 \%$ were determined to be $0.987,1.09$, and $0.786 \mathrm{MPa}$. The LPF80 data was negatively skewed, with the median closer to the top of the box (0.822 MPa), while the LPF60 and LPF100 data were more symmetrical (0.522 and $0.469 \mathrm{MPa}$, respectively). The interquartile range was high for all: $0.881,0.818$ and $0.702 \mathrm{MPa}$, for LPF60, LPF80 and LPF100, respectively. The average of the LPF80 data $(0.715 \mathrm{MPa}$ ) was also higher than that of the LPF60 and LPF100 resins ( 0.542 and $0.461 \mathrm{MPa}$, respectively). The LPF80 resin obtained the highest shear strength with its mean and median above the standard.

A trade-off was observed between the reactivity and the degree of branching of the lignin or phenolated lignin. The units of lignin (H-, G-, and S-units) are more branched than phenol, hence they have less reactive sites and a higher steric hindrance. Phenolated lignin at $60 \%$ substitution had the highest phenolic hydroxyl content (shown in

The highest phenolic hydroxyl contents were observed at a $\mathrm{NaOH} / \mathrm{PL}$ molar ratio of 0.378 for both substitution levels and phenolation temperatures of 110 and $80{ }^{\circ} \mathrm{C}$ at $60 \%$ and $80 \%$ substitution, respectively. The lower temperature required at $80 \%$ substitution could be from the higher incorporation of lignin with more branched units compared to phenol, which could have promoted bulk contact (Siddiqui et al., 2017), leading to faster initiation of the phenolation reaction. Furthermore, condensation of the phenolic hydroxyls could have occurred at too high temperatures and too long reaction times, which could then lead to a decrease in phenolic hydroxyl content (El Mansouri, Farriol \& Salvadó, 2006; Zhao et al., 2016).

Table 14), followed by phenolated lignin at $80 \%$, then by the unmodified lignin at $100 \%$ substitution. As expected, the more phenol incorporated, the more reactive sites available. Furthermore, the mid-IR spectra in Figure 17 showed that ether bonds were cleaved during phenolation, which contributed to less steric hindrance at $60 \%$ and $80 \%$ substitution. The worse performance of LPF60 compared to LPF 80 could be attributed to its lower degree of branching as well as a too high phenolic hydroxyl content. It has been reported that too high phenolic hydroxyl contents could promote non-covalent interactions which could have formed a crowded and stiff molecule (Zhang et al., 2013). Furthermore, at $80 \%$ the higher incorporation of more branched lignin could have positively contributed to a higher cross-link density compared to at $60 \%$ (Abdelwahab \& Nassar, 2011). The worse performance of LPF100 compared to LPF80 could therefore be attributed to more branched units hindering hydrogen bonding between the LPF adhesive and the plywood, resulting in reduced shear strengths (Moubarik et al., 2013). Also, the lower phenolic hydroxyl content and higher steric hindrance could lead to reduced cross-linking and hence shear strength.

Previous reports confirmed this trade-off: Abdelwahab \& Nassar (2011) also found that the adhesive strength increased with increasing lignin concentration up to $70 \%$, whereafter it started to decrease, which could be attributed to the positive contribution of lignin to the cross-link density, which will lead to better reinforcement structurally. Khan \& Ashraf (2007a) and Khan et al. (2004) also found that increasing lignin content increased the shear strength of the adhesive up to $50 \%$, whereafter it decreased due to the presence of excess lignin that did not form part of the cross-linked network. 


\subsubsection{Optimum substitution level and reaction conditions}

The optimum substitution level was determined to be at $68 \%$, with a phenolation temperature of $98.4{ }^{\circ} \mathrm{C}$, a $\mathrm{NaOH} / \mathrm{PL}$ molar ratio of 0.124 , and a F/PL molar ratio of 1 . These were determined by combining the data from the three CCD experimental results into a regression model (Equation 5 and Equation 6) to determine the substitution level that would give the highest shear strength. This was verified experimentally where a shear strength of $1.11 \pm 0.21 \mathrm{MPa}$ was produced.

The regression equation had an $R^{2}$ value of $39 \%$ and was not found to be significant with $p>0.10$ and $F<F_{\text {critical. }}$. The ANOVA results are shown in Table 15. Only a two factor CCD was used for LPF100 resins, which did not include the optimisation of the phenolation temperature as no phenolation occurred. Therefore, the regression model determining the optimum substitution level did not take the phenolation temperature into account, but only the substitution level, $\mathrm{NaOH} / \mathrm{PL}$ molar ratio, and F/PL molar ratio. This could be attributed to the contribution from the lack of fit of the LPF100 regression model (reported in section 4.2.1, with an $\mathrm{R}^{2}$ of $31 \%$ ), which had fewer data points. However, from the other regression models and literature knowledge, the parameters were expected and known to have a significant effect. The terms found significant was the quadratic term of the substitution level, the linear term of the F/PL ratio, and the linear term of the combined effects of the substitution level and the F/PL ratio.

Table 15. ANOVA of the combined effects of substitution level, NaOH/PL ratio, and F/PL ratio of all three CCDs.

\begin{tabular}{lccccc}
\hline & SS & df & MS & $\mathrm{F}$ & $\mathrm{p}$ \\
\hline (1) LPF percentage (L) & 0.0258 & 1. & 0.0258 & 0.58 & 0.45 \\
LPF percentage (Q) & $\mathbf{0 . 4 1 0 6}$ & $\mathbf{1 .}$ & $\mathbf{0 . 4 1 0 6}$ & $\mathbf{9 . 1 8}$ & $<0.01$ \\
(1) NaOH/PL molar ratio(L) & 0.0026 & 1. & 0.0026 & 0.06 & 0.81 \\
NaOH/PL molar ratio (Q) & 0.0288 & 1. & 0.0288 & 0.64 & 0.43 \\
(3) F/PL molar ratio (L) & $\mathbf{0 . 1 7 0 8}$ & $\mathbf{1 .}$ & $\mathbf{0 . 1 7 0 8}$ & $\mathbf{3 . 8 2}$ & $\mathbf{0 . 0 6}$ \\
F/PL molar ratio (Q) & 0.0300 & 1. & 0.0300 & 0.67 & 0.42 \\
1L by 2L & 0.0619 & 1. & 0.0619 & 1.38 & 0.25 \\
1L by 3L & $\mathbf{0 . 2 2 1 6}$ & $\mathbf{1 .}$ & $\mathbf{0 . 2 2 1 6}$ & $\mathbf{4 . 9 5}$ & $\mathbf{0 . 0 3}$ \\
2L by 3L & 0.0085 & 1. & 0.0085 & 0.19 & 0.67 \\
Error & 1.7446 & 39. & 0.0447 & & \\
\hline Lack of Fit & 0.8160 & 17. & 0.0480 & 1.14 & 0.38 \\
Pure Error & 0.9286 & 22. & 0.0422 & & \\
Total SS & 2.8516 & 48. & & & \\
\hline
\end{tabular}

When looking at the desirability plots in Figure 21, the shear strength only varied slightly (between 0.903 and $0.835 \mathrm{MPa}$ ) at a substitution level between $60 \%$ and $80 \%$, while it decreased sharply above $80 \%$ to a minimum of $0.450 \mathrm{MPa}$ at $100 \%$ substitution. This could indicate that although the substitution level was found to be very significant in the model, its influence was a lot more significant above $80 \%$ substitution, which could be explained by phenolation that occurred at $60 \%$ and $80 \%$ substitution which aided in increasing the shear strength, while no phenolation occurred at $100 \%$ substitution. 
LPF percentage
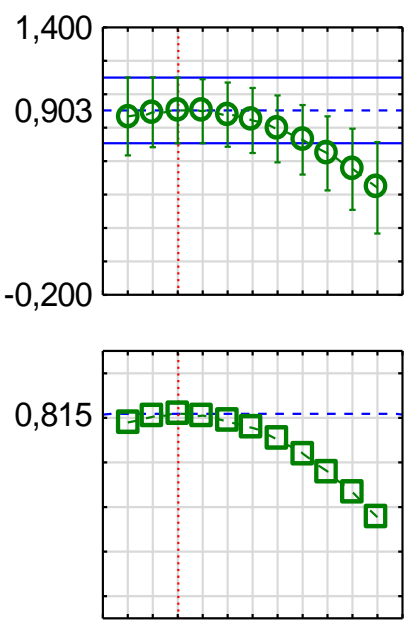

6068
$\mathrm{NaOH} / \mathrm{PL}$ molar ratio
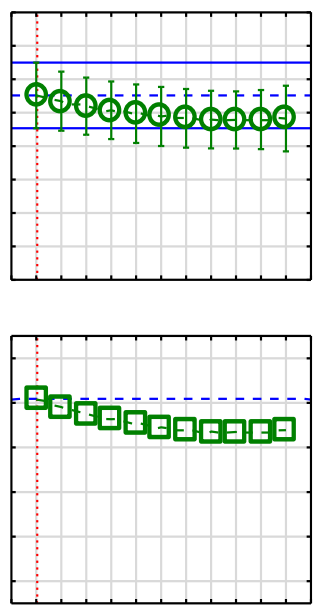

0,124
F/PL molar ratio
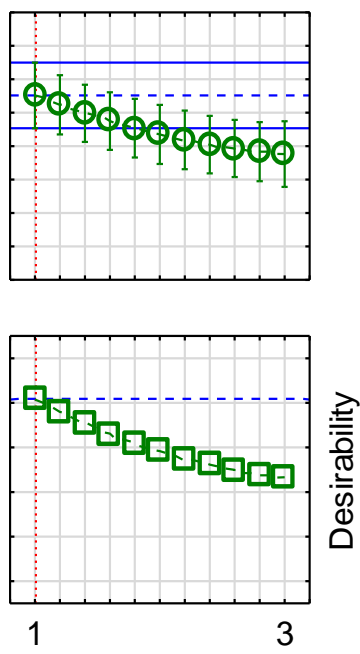

Desirability

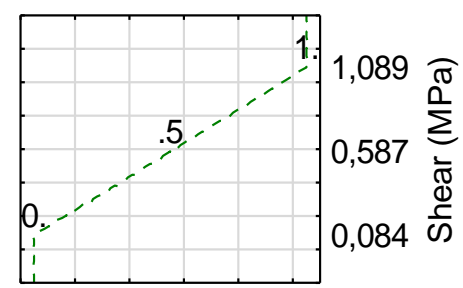

Figure 21. Desirability profile of the combined effects of the substitution level (from $60 \%$ to $100 \%$ ), the $\mathrm{NaOH} / \mathrm{PL}$ ratio, and the F/PL ratios. The green line represents the predicted curve, the red lines represent optimums and the blue lines the upper and lower bounds.

The shear strength from the desirability plots or when calculated (using optimum synthesis conditions: $68 \%$ substitution, $\mathrm{NaOH} / \mathrm{PL}$ molar ratio of $0.124, \mathrm{~F} / \mathrm{PL}$ molar ratio of 1 ) with the regression equation including significant and insignificant parameters, was determined to be $0.903 \pm 0.193 \mathrm{MPa}$, as calculated in Equation 5. When only taking the significant parameters into account, the maximum shear strength that could be obtained was calculated as $1.34 \mathrm{MPa}$ according to the regression model as shown in Equation 6. When verifying the regression model with experimental runs, the shear strength at the optimum conditions was determined to be $1.11 \pm 0.21 \mathrm{MPa}$, which correlated well. This was the highest shear strength obtained experimentally, with the next highest being LPF80_(1,-1,-1) with a shear strength of $1.09 \mathrm{MPa}$.

Equation 5:

Shear strength $(\mathrm{MPa})$

$$
\begin{aligned}
& =-0.312+0.0572(68 \%)-0.0005(68 \%)-5.6978\left(0.124 \frac{N a O H}{P L}\right)+7.8045\left(0.124 \frac{N a O H}{P L}\right) \\
& -0.8257\left(1 \frac{F}{P L}\right)+0.0576\left(1 \frac{F}{P L}\right)+0.0373\left(68 \% \times 0.124 \frac{N a O H}{P L}\right)+0.0060\left(68 \% \times 1 \frac{F}{P L}\right) \\
& +0.2424\left(0.124 \frac{N a O H}{P L} \times 1 \frac{F}{P L}\right)=\mathbf{0 . 9 0 3} \mathbf{~ M P a}
\end{aligned}
$$

Equation 6:

$$
\begin{aligned}
\text { Shear strength } & (\mathrm{MPa}) \\
& =-0.312+0.0572(68 \%)-0.0005(68 \%)^{2}-0.8257\left(1 \frac{F}{P L}\right)+0.0576\left(1 \frac{F}{P L}\right)^{2} \\
& +0.006\left(68 \% \times 1 \frac{F}{P L}\right)=\mathbf{1 . 3 4} \mathbf{~ M P a}
\end{aligned}
$$

Another regression equation was used and determined the optimum phenolation temperature to be $98.4^{\circ} \mathrm{C}$ at optimum conditions ( $68 \%$ substitution, $\mathrm{NaOH} / \mathrm{PL}$ molar ratio of $0.124, \mathrm{~F} / \mathrm{PL}$ molar ratio of 1 ). The regression 
equation was shown in Equation 7 with only the significant terms were included. This regression equation was derived from the combined effects of only the $80 \%$ and $60 \%$ substitution CCDs data. The substitution level only had a linear fit because there were only two substitution levels to include that had a phenolation temperature, while the other parameters had three levels and could have a quadratic fit as well.

Equation 7:

Shear strength $(\mathrm{MPa})=0.903 \mathrm{MPa}$

$$
\begin{gathered}
=-5.9753+0.0780(L P F \%)-2.0889\left(\frac{N a O H}{P L}\right)+21.3173(\mathrm{NaOH} / P L)^{2}+0.0848(T) \\
-0.0001(T)^{2}-0.2997\left(\frac{F}{P L}\right)+0.0348\left(\frac{F}{P L}\right)^{2}+0.0075\left(L P F \% \times \frac{N a O H}{P L}\right) \\
-0.0008(L P F \% \times T)+0.0041\left(L P F \% \times \frac{F}{P L}\right)-0.0508\left(\frac{N a O H}{P L} \times T\right) \\
+0.1471\left(\frac{N a O H}{P L} \times \frac{F}{P L}\right)-0.0030\left(T \times \frac{F}{P L}\right) \\
\therefore \boldsymbol{T}=\mathbf{9 8 . 4}{ }^{\circ} \mathbf{C}
\end{gathered}
$$

The regression model had an $\mathrm{R}^{2}$ value of $77.0 \%$, and the ANOVA results are shown in Table 16 . The $p$-value was found to be significant, while the F-value was not. The linear terms that had a significant effect included: the LPF percentage, the F/PL ratio, as well as the combined effects of the LPF percentage and temperature,

\begin{tabular}{|c|c|c|c|c|c|}
\hline & SS & $d f$ & MS & $\mathrm{F}$ & $\mathrm{p}$ \\
\hline (1) LPF percentage (L) & 0.24 & 1 & 0.24 & 10.69 & $<0.01$ \\
\hline (2) $\mathrm{NaOH} / \mathrm{PL}$ molar ratio(L) & 0.05 & 1 & 0.05 & 2.28 & 0.14 \\
\hline $\mathrm{NaOH} / \mathrm{PL}$ molar ratio (Q) & 0.13 & 1 & 0.13 & 5.80 & 0.02 \\
\hline (3) Temperature $\left({ }^{\circ} \mathrm{C}\right)(\mathrm{L})$ & 0.05 & 1 & 0.05 & 2.32 & 0.14 \\
\hline Temperature $\left({ }^{\circ} \mathrm{C}\right)(\mathrm{Q})$ & 0.01 & 1 & 0.01 & 0.36 & 0.56 \\
\hline (4) F/PL molar ratio (L) & 0.44 & 1 & 0.44 & 19.94 & $<0.01$ \\
\hline $\mathrm{F} / \mathrm{PL}$ molar ratio $(\mathrm{Q})$ & 0.01 & 1 & 0.01 & 0.30 & 0.59 \\
\hline $1 \mathrm{~L}$ by $2 \mathrm{~L}$ & 0.00 & 1 & 0.00 & 0.04 & 0.85 \\
\hline 1L by $3 \mathrm{~L}$ & 0.50 & 1 & 0.50 & 22.54 & $<0.01$ \\
\hline $1 \mathrm{~L}$ by $4 \mathrm{~L}$ & 0.03 & 1 & 0.03 & 1.52 & 0.23 \\
\hline $2 \mathrm{~L}$ by $3 \mathrm{~L}$ & 0.12 & 1 & 0.12 & 5.37 & 0.03 \\
\hline $2 \mathrm{~L}$ by $4 \mathrm{~L}$ & 0.00 & 1 & 0.00 & 0.11 & 0.74 \\
\hline $3 \mathrm{~L}$ by $4 \mathrm{~L}$ & 0.06 & 1 & 0.06 & 2.52 & 0.13 \\
\hline Lack of Fit & 0.48 & 16 & 0.03 & 7.37 & $<0.01$ \\
\hline Pure Error & 0.03 & 7 & 0.00 & & \\
\hline Error & 0.51 & 23 & 0.02 & & \\
\hline Total SS & 2.22 & 36 & & & \\
\hline
\end{tabular}
and the $\mathrm{NaOH} / \mathrm{PL}$ ratio and temperature. The only quadratic term that had a significant effect was the $\mathrm{NaOH} / \mathrm{PL}$ ratio.

Table 16. ANOVA of the effects from the substitution level (60\% to $80 \%$ ), $\mathrm{NaOH} / \mathrm{PL}$ ratio, and F/PL ratio on the shear strength. 


\subsection{LPF resins characterisation}

\subsubsection{Mid-IR spectra of the LPF resins}

The mid-IR spectra of the LPF resins are shown in Addendum C, and the band assignments are tabulated in Table 17. All the resins had a broad peak from 3400 to $3200 \mathrm{~cm}^{-1}$ attributed to the stretching of hydroxyl groups. All the resins also had a peak around $1390 \mathrm{~cm}^{-1}$ attributed to the vibration of phenolic hydroxyl groups. All the samples peaked at $1600 \mathrm{~cm}^{-1}$ typical of a lignin aromatic structure (El Mansouri et al., 2018).

Table 17. Mid-IR spectra band assignments of the LPF resins.

\begin{tabular}{|c|c|c|}
\hline Band $\left(\mathrm{cm}^{-1}\right)$ & Assignment & Reference \\
\hline 3400 to 3200 & $\mathrm{O}-\mathrm{H}$ stretching vibration & (Hussin et al., 2017) \\
\hline 2927 & Asymmetric $\mathrm{CH}_{2}$ vibration & (Hussin et al., 2017) \\
\hline 2850 & Out of phase stretching vibration of $-\mathrm{CH}_{2}$ - alkane & (Hussin et al., 2017) \\
\hline 1600 to 1500 & $\mathrm{C}=\mathrm{C}$ stretching vibration in benzene ring & (Hussin et al., 2017) \\
\hline 1500 to 1400 & Deformation vibration of $\mathrm{C}-\mathrm{H}$ in $-\mathrm{CH}_{2}-$ & (El Mansouri et al., 2018) \\
\hline 1480 to 1450 & $\mathrm{CH}$ deformation mode in $-\mathrm{CH}_{2}$ - bridge & (Hussin et al., 2017) \\
\hline 1400 to 1000 & C-O vibrations of ether bonds & (Alonso et al., 2001) \\
\hline 1390 & Phenolic $\mathrm{OH}$ in plane & (Hussin et al., 2017) \\
\hline 1360 to 1350 & $\mathrm{C}-\mathrm{O}$ stretching in $\mathrm{S}$ unit & (El Mansouri et al., 2018) \\
\hline 1270 to 1220 & $\mathrm{C}-\mathrm{O}(\mathrm{H})+\mathrm{C}-\mathrm{O}(\mathrm{Ar})$ (phenolic $\mathrm{OH}$ and ether in $\mathrm{S}$ and $\mathrm{G}$ ) & $\begin{array}{l}\text { (Hussin et al., 2017; El } \\
\text { Mansouri et al., 2018) }\end{array}$ \\
\hline 1160 & $\mathrm{C}-\mathrm{O}$ ether stretch in methylol groups (H-units) & (Moubarik et al., 2013) \\
\hline 1154 & $\mathrm{C}-\mathrm{H}$ stretching in S-units & (El Mansouri et al., 2018) \\
\hline 1125 & $\mathrm{C}-\mathrm{H}(\mathrm{Ar})$ in-plane deformation (G-units) & (Moubarik et al., 2013) \\
\hline 1115 & $\mathrm{C}-\mathrm{H}(\mathrm{Ar})$ in-plane deformation (S-units) & (Hussin et al., 2017) \\
\hline 1050 & $\mathrm{C}-\mathrm{O}$ ether stretching in methylol groups & (Hussin et al., 2017) \\
\hline 1020 & $\mathrm{C}-\mathrm{O}$ stretch in methylol C-OH and C-O (Ar) & (Li et al., 2016) \\
\hline 850 to 750 & Tri-substituted benzene structure & (El Mansouri et al., 2018) \\
\hline 835 & CH out-of-plane, para-substituted (G-units) & (Hussin et al., 2017) \\
\hline 760 & CH out-of-plane, ortho-substituted & (Hussin et al., 2017) \\
\hline 694 & Mono-substituted benzene ring structure & (Zhao et al., 2016) \\
\hline
\end{tabular}

During the synthesis reaction, formaldehyde was attached to the available reactive sites on the phenolated lignin as methylol groups. During synthesis, the methylol groups condensed to form methylene bridges and dimethyl ether bridges (Yang et al., 2014). Therefore, an increase in methylene peak intensities indicated successful polycondensation: asymmetric methylene vibration occurred at $2927 \mathrm{~cm}^{-1}$, out of phase stretching vibration of the $-\mathrm{CH}_{2}$ - alkane occurred around $2850 \mathrm{~cm}^{-1}$, and methylene $\mathrm{C}-\mathrm{H}$ bending occurred at 1475 to 1450 $\mathrm{cm}^{-1}$ (Hussin et al., 2017). 
During the addition reaction, formaldehyde is introduced into the free ortho- and para-positions of the aromatic rings. Therefore a decrease in G-units at the shoulder bands at 1272 and $835 \mathrm{~cm}^{-1}$, as well as a decrease in $\mathrm{H}$-units at $1160 \mathrm{~cm}^{-1}$, indicates that the addition of formaldehyde onto the aromatic ring was successful (Wang \& Chen, 2014; Hussin et al., 2017). The bands between 850 to $750 \mathrm{~cm}^{-1}$ indicated trisubstituted benzene rings and confirmed the formation of a cross-linked three-dimensional network structure (EI Mansouri et al., 2018). The band around $835 \mathrm{~cm}^{-1}$ indicated C-H out-of-plane para-substituted benzene, while at $760 \mathrm{~cm}^{-1}$ it indicated $\mathrm{C}$-H out-of-plane ortho-substituted benzene (Hussin et al., 2017).

\subsubsection{Comparing LPF100 resins from the screening experiments using different lignins}

The mid-IR spectra of the centre point runs of the LPF resins made from different lignins are shown in Addendum C - Mid-IR spectra of the LPF resins. Their shear strengths (as reported in Addendum B and previously in Figure 13 and Figure 20) ranked from high to low, were: $0.529>0.430>0.409>0.280>0.145$, for the S-A-P LPF80_(0,0,0), S-A-P LPF100_(0,0), S-A-P LPF60_(0,0,0), L-S LPF100_(0,0), and L-S-D LPF100_(0,0) resins, respectively.

A high hydroxyl content in the uncured resin foreshadowed a high shear strength in the adhesive. All of the samples also showed the presence of hydroxyl groups at 3400 to $3200 \mathrm{~cm}^{-1}$ (El Mansouri et al., 2018), where the S-A-P resin had higher peak intensities than the lignosulphonate resins. The peaks at $1220 \mathrm{~cm}^{-1}$ indicated the presence of $\mathrm{C}-\mathrm{O}$ stretching of the phenolic $\mathrm{OH}$ on the S- and G-units (Hussin et al., 2017). No peak was present for the L-S-D LPF100_(0,0) resin, while only a weak intensity peak was present for the L-S LPF100_(0,0) resin. Furthermore, the S-A-P LPF80_(0,0,0) had the highest hydroxyl concentration, followed by LPF60_(0,0,0) and then the LPF100_(0,0). These findings correlated to the rank of their shear strengths.

All three of the LPF100_(0,0) resins showed peaks at $1115 \mathrm{~cm}^{-1}$ attributed to $\mathrm{C}-\mathrm{H}$ in-plane vibrations of S-units (Hussin et al., 2017), while fewer S-units were present due to the incorporation of phenol in LPF60_(0,0,0) and LPF80_(0,0,0) resins. The intensity of this peak was higher for L-S-D than L-S LPF100_(0,0) resin, which could be attributed to L-S-D having more of the higher molecular weight S-units after dialysis as found in section 4.1.2.

A high methylene bond intensity in the LPF resin foreshadowed a high shear strength. The peaks from 1480 to $1450 \mathrm{~cm}^{-1}$ indicated the presence of methylene bonds formed during condensation of methylol groups in the methylolation reaction (Alonso et al., 2001; El Mansouri et al., 2018). The S-A-P LPF60_(0,0,0) and LPF80_(0,0,0) resins showed intense peaks around $1478 \mathrm{~cm}^{-1}$, which showed that more methylene bonds formed during the condensation reaction compared to the LPF100_(0,0) resins. For the LPF100_(0,0) resins, SA-P had the highest peak in this region, followed by L-S, the L-S-D. The intensities of these peaks corresponded to the trend observed in the shear data.

\subsubsection{PCA of the LPF resins}

A principal component analysis was performed using the mid-IR data from the resins characterised to identify how the chemical structure of the resins influenced the shear strength. The PCA grouped resins with similar peaks in the spectra which aided in identifying similarities and differences, as the spectra are complex and there were many to compare. The resins formed 7 different clusters as shown in the score plot in Figure 22. 
Overall, the resins had similar structures with small differences between the clusters that were identified. The principal components PC1, PC2, PC3, and PC4 again described $61.4 \%, 16.1 \%, 9.2 \%$ and $4.4 \%$ of the variability in the data, respectively, as shown in Figure 10. All the resins were made with S-A-P lignin unless otherwise indicated. The resins were colour-coded as follows: blue resembled the centre point runs of the S-A-P resins. Red and green represented the bad and good performing S-A-P resins, respectively. Purple represented the LPF100_(0,0) resins made with lignosulphonate, while yellow represented the S-A or S-A-P LPF68 resins made at optimum conditions (phenolation temperature of $98.4^{\circ} \mathrm{C}, \mathrm{NaOH} / \mathrm{PL}$ molar ratio of 0.124 , and F/PL molar ratio of 1).

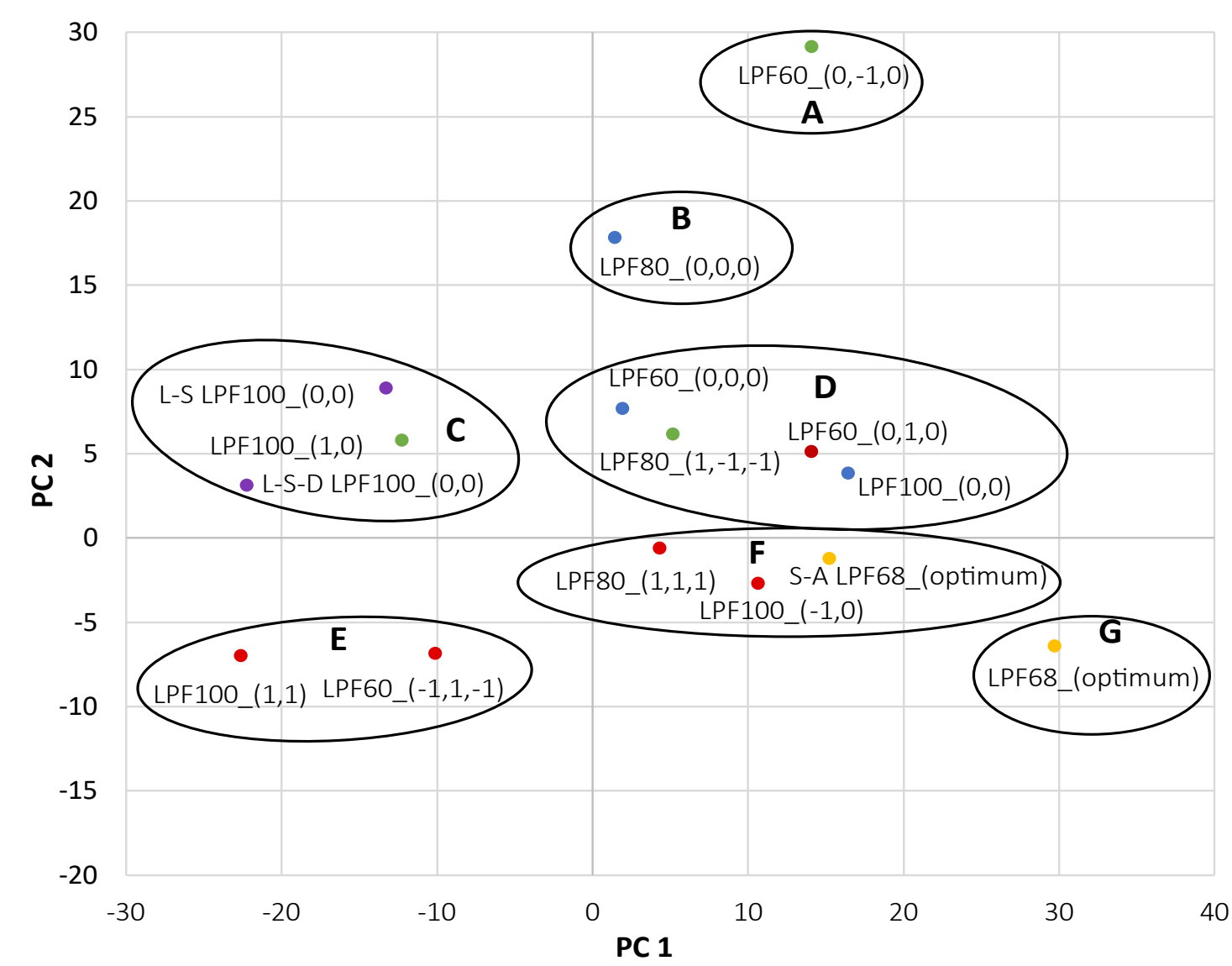

Figure 22. Scores plot of PC1 and PC2 from the mid-IR data of the different LPF resins.

The presence of G-units indicates that a reactive site is still available for methylol to react with to form a methylene bridge. Furthermore, a decrease in G-units could also indicate that successful addition of formaldehyde happened at the reactive site, or that cross-linking occurred during the condensation reaction. Cluster D, F and G had G-units indicated in their IR spectra at $1279 \mathrm{~cm}^{-1}$. Cluster C did not indicate any peaks attributed to G-units present at 1272 or $835 \mathrm{~cm}^{-1}$. This could indicate that addition at the available reactive sites was successful for the LPF100 resins, but further cross-linking did not occur which could be attributed to higher steric hindrance at $100 \%$ substitution.

A high hydroxyl content in the LPF resins before curing would be favourable for further cross-linking and hence contribute positively to the shear strength. Cluster B only contained the LPF80_(0,0,0) resin, and its separation could be due to the large peak at 3400 to $3200 \mathrm{~cm}^{-1}$ attributed to the total hydroxyls present. Cluster E 
contained the two samples with the lowest total hydroxyl group content ( 3400 to $3200 \mathrm{~cm}^{-1}$ ) namely LPF100_(1,-1) and LPF80_(-1,1,-1), which could have contributed to their low shear strengths produced.

Ether bonds formed during condensation of methylol groups indicate that cross-linking occurred and will hence contribute positively to the shear strength produced. Cluster B containing S-A-P LPF80_(0,0,0) has an overlap of the two peaks at 987 and $1030 \mathrm{~cm}^{1}$, which indicated the presence of $\mathrm{C}-\mathrm{O}$ vibration of ether bands (Alonso et al., 2001). Cluster $C$ had no peaks at 1150 to $1050 \mathrm{~cm}^{-1}$ attributed to the stretching of $\mathrm{C}-\mathrm{O}$ bonds in alkyl-substituted ethers which contributed to their low shear strengths.

Methylene bridges are strong $\mathrm{C}-\mathrm{C}$ bonds linking different lignin units, where the formation of these bonds before the curing reaction would indicate that cross-linking occurred, which would be favourable if these cross-linked networks were successfully embedded into the plywood. A high degree of cross-linking would then lead to a more thermally stable resin and yield a high shear strength. Cluster $A$ and $G$ indicated a high methylene content at $1472 \mathrm{~cm}^{-1}$, attributed to the bending of methylene $\mathrm{C}-\mathrm{H}$ bonds. Both samples, LPF60_(0,$1,0)$ and LPF68_(-1,-1,0), had a very high shear strength.

In Figure 23 the absorbances of the methylene peaks (at $1470 \mathrm{~cm}^{-1}$ ) in the mid-IR spectra of the LPF resins before hot-pressing were plotted against the shear strength obtained. It was observed that a high methylene bridge content already formed in the resin before hot-pressing led to high shear strengths produced. Therefore, the high shear strength performance of the LPF resins was attributed to a high degree of crosslinking in the resin, while the resins that produced lower shear strengths lacked a good degree of cross-linking.

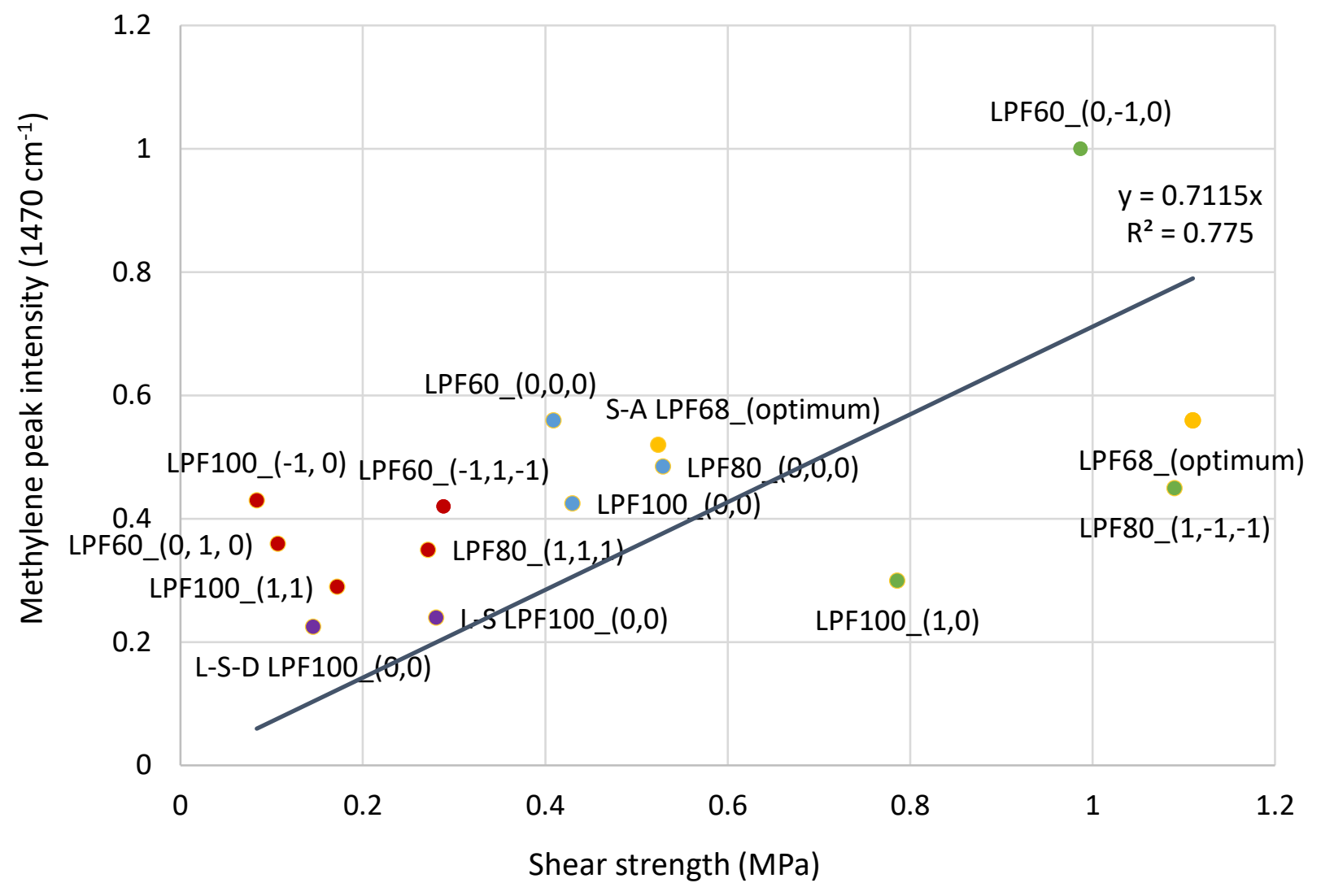

Figure 23. Scatter plot correlating the methylene bridges formed at $1470 \mathrm{~cm}^{-1}$ from the mid-IR data to the shear strength of the LPF resins. 


\subsubsection{TGA of the LPF resins}

The thermal stability of the LPF resins was investigated using TGA to determine at which temperatures they start to degrade and to determine the maximum temperature at which hot-pressing could be applied. The resins had less than $10 \%$ weight loss below $200{ }^{\circ} \mathrm{C}$, hence a hot-pressing temperature below $200{ }^{\circ} \mathrm{C}$ would be required. The TG curves displayed the weight loss of each sample as temperature increased, while the first derivative DTG curves displayed the corresponding rate of weight loss, with a peak on the DTG curve representing the maximum thermal decomposition temperature that could be used to compare the thermal stability of the different LPF resins (Tejado et al., 2007). A higher frequency of strong inter-unit linkages is more resistant to thermal degradation (Pang et al., 2017) and would thus be favourable for LPF resins, as the higher degree of cross-linking contributes to high shear strength and thermal stability of the resin if the lignin macromolecule is successfully embedded (Pang et al., 2017). The weight loss that occurred below $100{ }^{\circ} \mathrm{C}$ was attributed to the release of low molecular weight components (such as phenol, formaldehyde, solvents, etc.) and volatiles (Alonso et al., 2011). Thereafter, degradation of the LPF resins then occurred in three steps, namely post-curing, thermal reforming, and ring stripping (Yang et al., 2019).

\subsubsection{LPF100_(0,0) resins synthesised using different types of lignin}

The LPF100_(0,0) resin made with S-A-P had a higher thermal stability than with L-S and L-S-D. The maximum thermal degradation of the S-A-P resin occurred at $275{ }^{\circ} \mathrm{C}$, followed by L-S and L-S-D at 270 and $246{ }^{\circ} \mathrm{C}$, respectively, as shown in Figure 24. This correlated with the shear strength results in section 4.1.4, where a higher shear strength corresponded with a higher thermal stability.

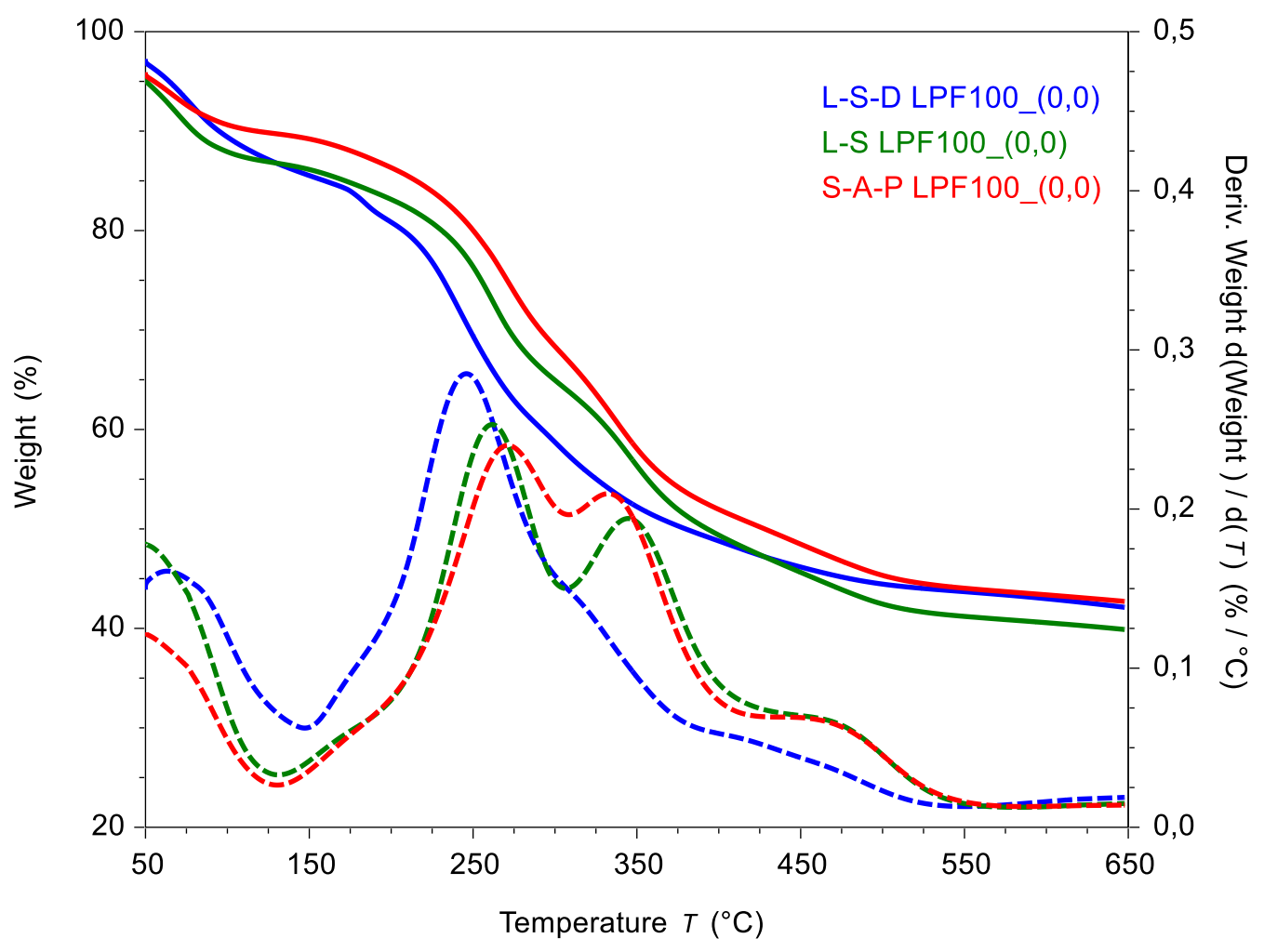

Figure 24. TGA and DTG graphs of the LPF100_(0,0) resins synthesised with S-A-P, L-S and L-S-D. 
The S-A-P and L-S LPF100_ $(0,0)$ resins had peaks on the DTG curve in the second degradation endotherm around $350^{\circ} \mathrm{C}$. These peaks were not observed in the lignin samples in Figure 12 , which indicated that stable cross-linking bonds were formed. The peak between 300 and $400{ }^{\circ} \mathrm{C}$ was attributed to the degradation of the polymer, with the lower molecular weight components degrading first, which included cleavage of the side chains on the lignin (Khan \& Ashraf, 2007). The peaks around $454{ }^{\circ} \mathrm{C}$ indicated methylene bonds (Zhang et al., 2013), which confirmed that methylol groups did condense to form cross-linking methylene bridges in the $\mathrm{S}$ A-P and L-S resins. This agreed with the mid-IR spectra (section 4.3.1.1) where the LPF100_(0,0) resins made with S-A-P had the highest methylene content at $1460 \mathrm{~cm}^{-1}$, followed by L-S, then L-S-D. From the mid-IR lignin characterisation (section 4.1.2) and resin characterisation (section 4.3.1.1), L-S-D indicated more S- and Gunits compared to L-S and S-A-P, thus its lower reactivity and higher steric hindrance attributed to its low degree of cross-linking.

\subsubsection{S-A-P LPF resins that produced the highest shear strength at each substitution level}

Figure 25 shows the TGA and DTG curves of the S-A-P LPF resins that produced the highest shear strengths at different substitution levels. LPF68_(optimum) was found to be the most thermally stable as it had the highest maximum thermal degradation at $382{ }^{\circ} \mathrm{C}$, and it also had the highest weight residue throughout. LPF60_(0,-1,0) also showed good thermal stability, with its highest maximum thermal degradation at $367^{\circ} \mathrm{C}$, followed by LPF80_(1,-1,-1) at $343^{\circ} \mathrm{C}$ and then LPF100_(1,0) at $264^{\circ} \mathrm{C}$.

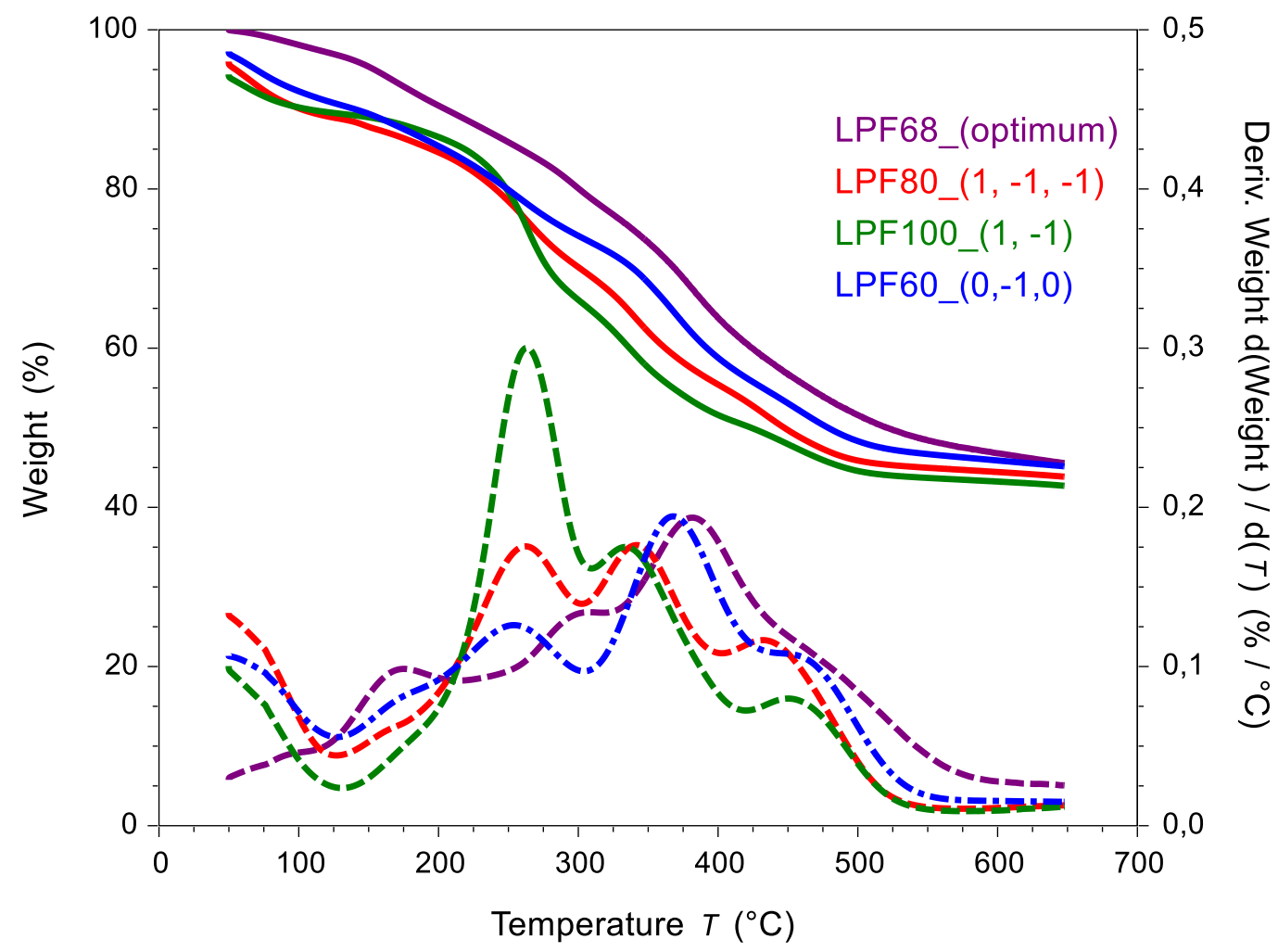

Figure 25. TGA and DTG graphs of the S-A-P LPF resins that produced the highest shear strength at each substitution level. 
The higher thermal stability of the LPF resins at substitution levels below $100 \%$, could be attributed to the phenolation reaction that already cleaved weak bonds, while LPF100_(1,0) had a high weight loss at low temperatures. Furthermore, only a small peak was observed for LPF100_(1,0) around $450{ }^{\circ} \mathrm{C}$ which indicates that not a lot of methylene bridges formed compared to the other resins, which agreed with the findings from Figure 23 , where the methylene bridges in the mid-IR data at $1470 \mathrm{~cm}^{-1}$ were correlated with the shear strengths of the LPF resins. The methylene bridges around $450{ }^{\circ} \mathrm{C}$ again correlated directly with the shear strengths produced (reported in Addendum B).

\subsubsection{Comparing S-A-P LPF resins synthesised at different conditions for each substitution level}

The incorporation of phenol by the phenolation reaction formed resins with higher thermal stabilities, compared to at $100 \%$ substitution where no phenol was incorporated. As seen in Figure 26 to Figure 28 below, the LPF100 resins had their maximum thermal degradation around $250{ }^{\circ} \mathrm{C}$, while the LPF80 and LPF60 resins had their maximum degradation around $350{ }^{\circ} \mathrm{C}$. The lower reactivity at higher lignin incorporation at $100 \%$ substitution formed fewer cross-linked bridges (from the mid-IR data in Figure 23) and hence fewer branched polymeric structures which lead to degradation at lower temperatures. Furthermore, the cleavage of weak ether bonds could also lead to more weight loss at lower temperatures in LPF100, which were cleaved already during phenolation at $60 \%$ and $80 \%$ substitution. Some samples were identified from each CCD at each substitution level which resembled the best performing (marked green), the centre point (marked blue), the worst performing (marked red) and another bad performing (marked purple) resin, to correlate the thermal stability, degree of cross-linking and shear strength.

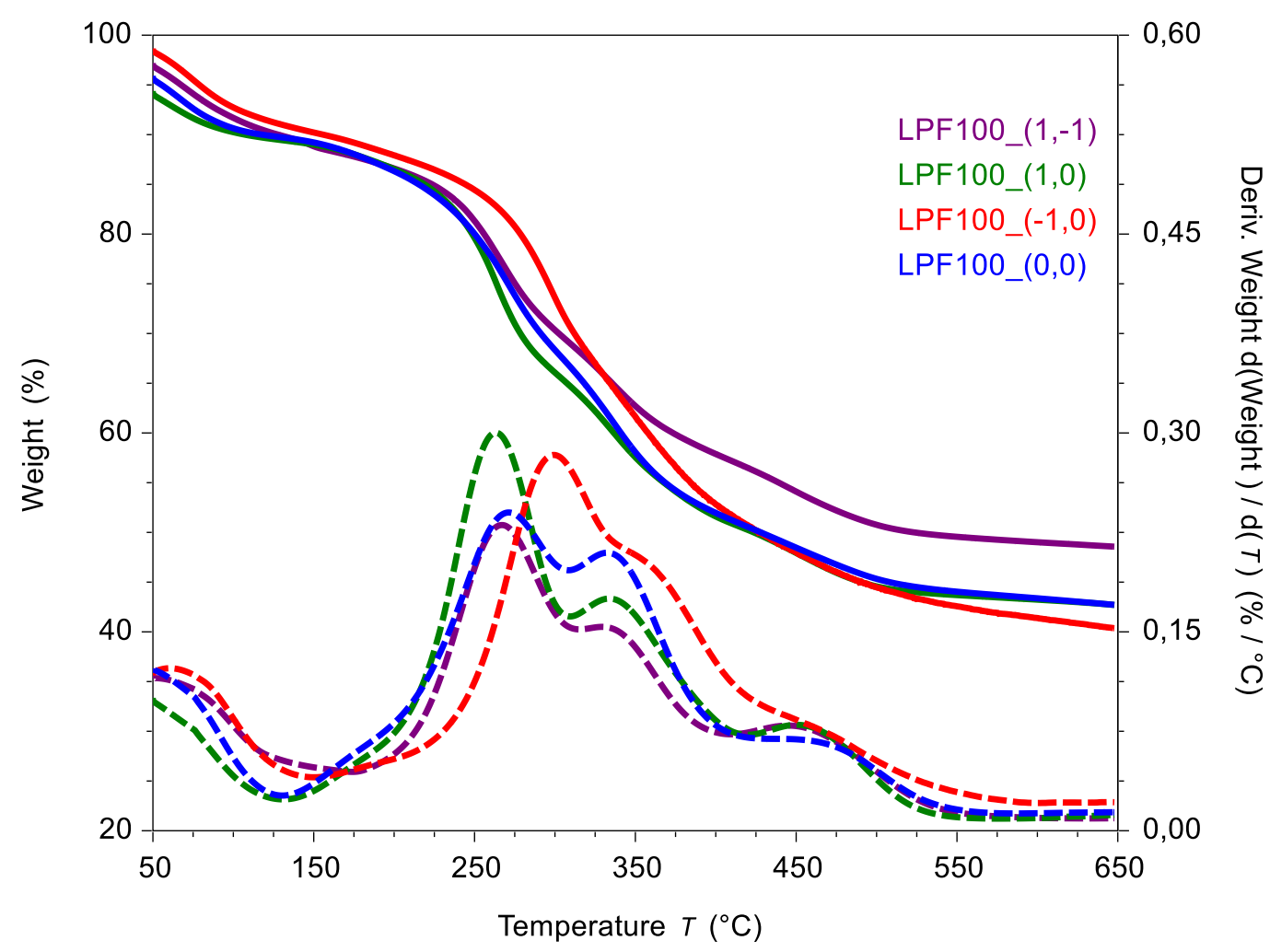

Figure 26. TGA and DTG graphs of the LPF100 resins made with S-A-P lignin phenolated and synthesised at different reaction conditions. 
Figure 26 compares the LPF100 resins made with S-A-P lignin at different phenolation and synthesis conditions. LPF100_(-1,0) had the highest maximum thermal degradation temperature at $300^{\circ} \mathrm{C}$, while the other samples experienced their maximum degradation from 265 to $275^{\circ} \mathrm{C}$. This could be attributed to the lower $\mathrm{NaOH} / \mathrm{PL}$ and F/PL molar ratios compared to the other samples, which caused a slower completion of the curing reaction (Siddiqui et al., 2017). LPF100_(-1,0) had no peaks at the second and third degradation endotherms, indicating that a low degree of cross-linking occurred, which explained its weak shear strength. LPF100_(1,0) and LPF100_(0,0) showed similar thermal properties, both with a peak around $450{ }^{\circ} \mathrm{C}$ indicating that cross-linking occurred, and methylene bridges were formed.

Figure 27 compares the LPF80 resins made with S-A-P lignin at different phenolation and synthesis reaction conditions. All three of the samples had degradation peaks at similar temperatures. LPF80_(1,1,1) experienced a high rate of degradation around $350{ }^{\circ} \mathrm{C}$, attributed to the degradation of the polymer, with the lower molecular weight components degrading first, which included cleavage of the side chains on the lignin (Khan \& Ashraf, 2007). LPF80_(1,-1,-1) had the highest shear strength and a prominent peak at $450{ }^{\circ} \mathrm{C}$ indicating a good degree of cross-linking from methylene bridges formed.

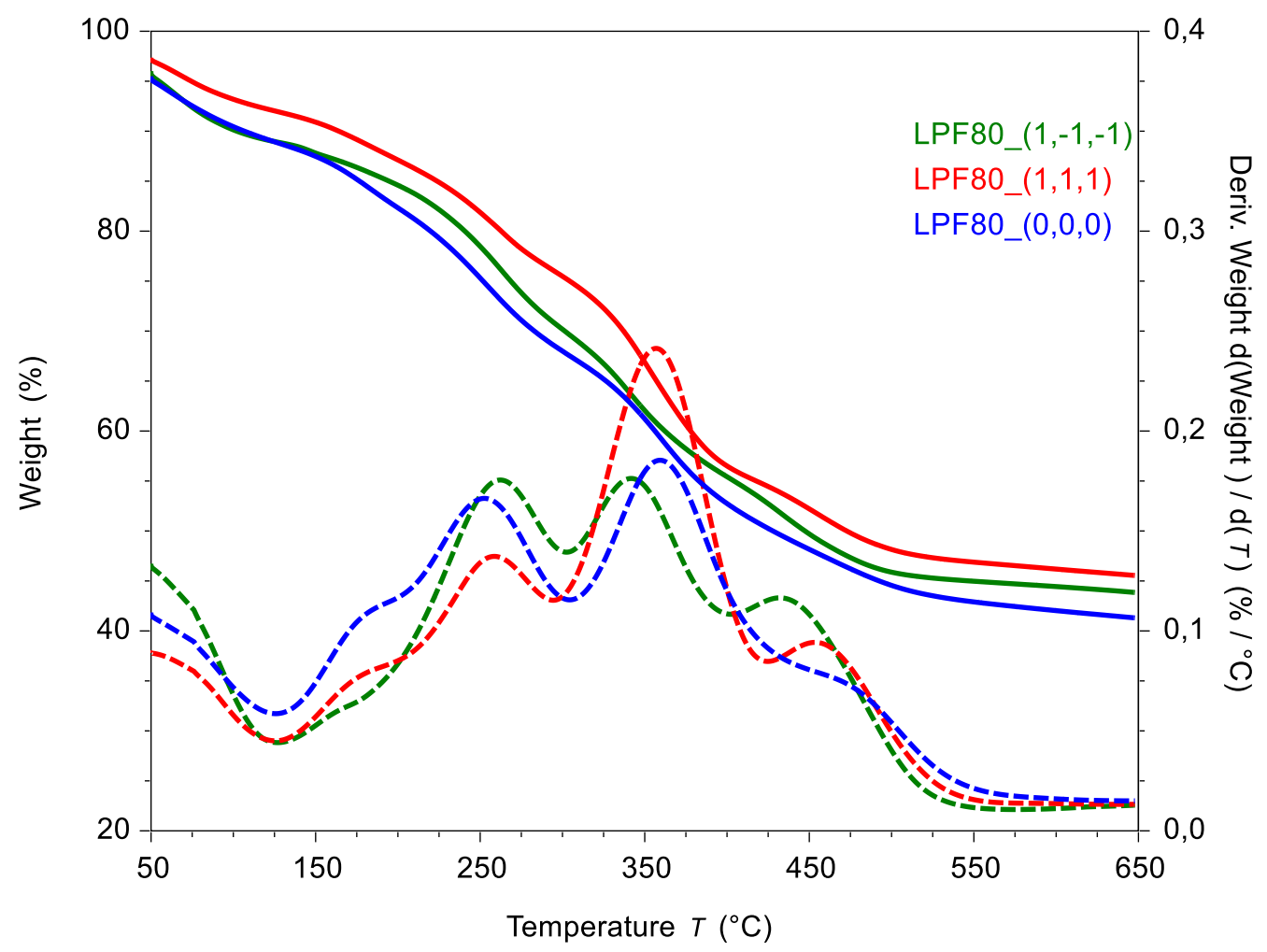

Figure 27. TGA and DTG graphs of the LPF80 resins made with S-A-P lignin phenolated and synthesised at different reaction conditions.

Figure 28 compares the LPF60 resins made with S-A-P lignin at different phenolation and synthesis reaction conditions. All of the samples had a similar maximum degradation peak around $365^{\circ} \mathrm{C}$. LPF60_(-1,1,-1) has a high degradation peak at $166{ }^{\circ} \mathrm{C}$ with around $10 \%$ weight loss, which could be attributed to the evaporation of water and formaldehyde released by the condensation of methylol groups (Zhang et al., 2013), and 
unreacted formaldehyde from the addition reaction as the F/PL molar ratio of three used which was at the upper-bound. LPF60_(0,-1,0) had the most prominent peak at $450^{\circ} \mathrm{C}$, attributed to methylene bridges formed. The weight residue at $595{ }^{\circ} \mathrm{C}$ of LPF60_(-1,1,-1) was also the lowest, followed by the LPF60_(0,0,0) and then LPF60_(0,-1,0) and LPF60_(0,1,0), which correlated to their shear strengths and confirmed a high degree of cross-linking leads to a high shear strength.

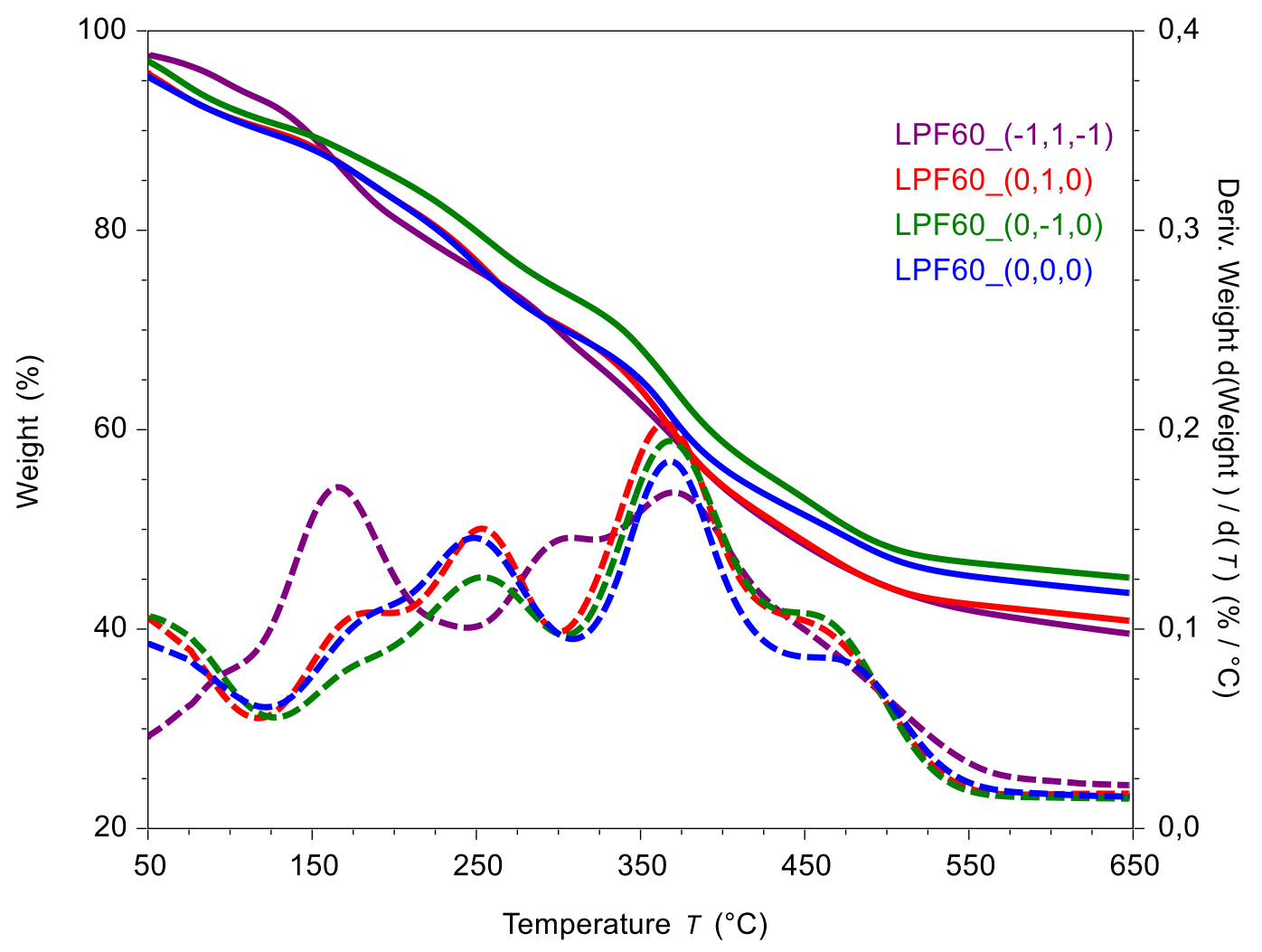

Figure 28. TGA and DTG graphs of the LPF60 resins made with S-A-P lignin phenolated and synthesised at different reaction conditions.

\subsubsection{Free formaldehyde content and $\mathrm{pH}$ of the LPF resins}

Table 18 reports the $\mathrm{pH}$ values and free formaldehyde contents for the optimum and centre point shear runs of each CCD with S-A-P lignin. The GB/T 14732-2013 standard specified the pH should be larger than 7 and the free formaldehyde content should be equal to or below $0.30 \mathrm{wt} \%$. The $\mathrm{pH}$ values of the LPF resins should range from a pH value of 8 to 13 to avoid lignin precipitation and maintain a high water resistance (Tonge, 2007). The $\mathrm{pH}$ values of the resins produced from the CCD runs were all above a $\mathrm{pH}$ value of 7 and were well within this range, with the lowest $\mathrm{pH}$ of 8.5 and the highest $\mathrm{pH}$ of 11.4 for resins synthesised with S-A-P.

The free formaldehyde contents of the resins which produced the highest shear strengths adhered to the standard requirements ranging from $0.177 \%$ to $0.298 \%$. This verified that at optimal conditions most of the formaldehyde reacted and attached to the phenolic lignin structures, leaving a low residual formaldehyde content. Standard deviations for the free formaldehyde contents ranged between $0.002 \mathrm{wt} \%$ and $0.033 \mathrm{wt} \%$. At the centre points, the LPF80_ $(0,0,0)$ and LPF60_(0,0,0) resins failed to adhere to the free formaldehyde standard, indicating that the conditions were not favourable for most of the formaldehyde to react, leading to 
a high free formaldehyde content. These resins did not show G-unit peaks in their mid-IR spectra at $835 \mathrm{~cm}^{-1}$ (shown in Addendum C), which showed that there were no reactive sites for the residual formaldehyde to attach during the addition reaction, resulting in a high free formaldehyde content, while a G-unit peak was observed for the LPF100_(0,0) resin. None of these centre point resins showed H-unit peaks at $110 \mathrm{~cm}^{-1}$, indicating that formaldehyde had attached to the reactive sites.

Table 18. Free formaldehyde content, shear strength and $\mathrm{pH}$ values of the centre point and optimum LPF resin samples.

\begin{tabular}{lccccc}
\hline Resin ID & $\begin{array}{c}\text { Free } \mathbf{C H}_{2} \mathbf{O} \\
(\mathbf{w t} \%)\end{array}$ & $\mathbf{C H}_{2} \mathbf{O} \leq \mathbf{0 . 3 0 \%}$ & pH value & $\begin{array}{c}\text { Shear strength } \\
\text { (MPa) }\end{array}$ \\
\hline Highest & LPF100_(1,-1) & 0.298 & pass & 9.9 & 0.786 \\
shear & LPF80_(1,-1,0) & 0.117 & pass & 11.4 & 1.09 \\
strength & LPF60_(0,-1,1) & 0.178 & pass & 10.5 & 0.987 \\
& LPF68_(optimum) & 0.284 & pass & 9.0 & 1.11 \\
\hline Centre & LPF100_(0,0) & 0.291 & pass & 9.1 & 0.430 \\
points & LPF80_(0,0,0) & 0.837 & fail & 10.4 & 0.529 \\
runs & LPF60_(0,0,0) & 0.554 & fail & 10.4 & 0.409 \\
\hline
\end{tabular}

\subsubsection{Performance valuation of the resin in particleboard manufacture}

High-density particleboard was made with the S-A-P LPF68_(optimum) resin and it proved to have good resistance to moisture and confirmed the good shear strength performance as observed with the plywood. The density of the particleboards produced was around $900 \mathrm{~kg} / \mathrm{m}^{3}$ with an area and thickness of $0.09 \mathrm{~m}^{2}$ and $6 \mathrm{~mm}$, respectively. The particleboards were produced and tested according to the ASTM D1037-12 standard, and the results were tabulated in Table 19. The ANSI A208.1-1999 standard for high-density $\left(>800 \mathrm{~kg} / \mathrm{m}^{3}\right)$ particleboards specifies a $\mathrm{MOR} \geq 23.5 \mathrm{MPa}$ and $\mathrm{MOE} \geq 2750 \mathrm{MPa}$. The particleboard made with LPF68_(optimum) exceeded these MOR and MOE specifications. A MOR and MOE of 40 and 3209 MPa were obtained, respectively. The TS and WA were low and determined to be $13.5 \%$ and $37.2 \%$, respectively. Another board was made with commercial Bondtite (a urea-based resin used for interior application) mixed with hexamine as a cross-linker, used only as a reference board. The TS and WA were determined to be $61.8 \%$ and $73.3 \%$, respectively. The lower moisture resistance of this urea-based resin was expected. The MOR and MOE were 27 and $3592 \mathrm{MPa}$, respectively. This confirmed that this S-A-P LPF68 resin had great strength and moisture resistant properties.

Table 19. Moisture content (MC), thickness swelling (TS), water absorption (WA), modulus of rupture (MOR), and modulus of elasticity (MOE) of the particleboard produced with LPF68 and Bondtite (Standard deviations reported in brackets).

\begin{tabular}{lccccc}
\hline & MC of dry boards (\%) & TS (\%) & WA (\%) & MOR (MPa) & MOE (MPa) \\
\hline \multirow{2}{*}{ LPF68 } & 8.41 & 13.5 & 37.2 & 40 & 3209 \\
& $(0.8)$ & $(5.56)$ & $(4.74)$ & $(2.47)$ & $(323)$ \\
\multirow{2}{*}{ Bondtite } & 10.9 & 61.8 & 73.3 & 27 & 3592 \\
& $(0.97)$ & $(6.60)$ & $(7.61)$ & $(3.79)$ & $(191)$ \\
\hline
\end{tabular}


The LPF68_(optimum) particleboard produced performed better than those previously reported, and at higher substitution levels. Çetin and Özmen (2002) made medium-density $\left(650 \mathrm{~kg} / \mathrm{m}^{3}\right)$ particleboards using a control, LPF20, and LPF30 adhesives, respectively, using organosolv lignin. The MOEs obtained were 2175, 2267 and $2224 \mathrm{MPa}$ and the corresponding MORs were 14, 16 and $12.7 \mathrm{MPa}$, respectively. Thickness swelling after 24 hours of $13.5 \%, 12.9 \%$, and $14.7 \%$, and water absorption of $75.0 \%, 73.2 \%$, and $72.9 \%$, respectively, was also reported. Iwakiri et al. (2019) produced particleboards with a LPF10 adhesive $\left(750 \mathrm{~kg} / \mathrm{m}^{3}\right.$, pressed for 10 minutes) and a LPF12 adhesive (950 kg/m $\mathrm{m}^{3}$, pressed for 15 minutes). The MOR ranged from 11.9 to $25.4 \mathrm{MPa}$, and the MOE ranged from 1782 to $3083 \mathrm{MPa}$. Stücker et al. (2016) produced particleboards with alkaline extraction lignin and enzymatic hydrolysis lignin at $20 \%$ substitution. The MOR and MOE values produced was around $16 \mathrm{MPa}$ and $2500 \mathrm{MPa}$ for both the adhesives made with the different lignins. The thickness swelling was around $20 \%$ for both, while the water absorption was $71.4 \%$ and $74.1 \%$ for the respective lignins. The higher density could have contributed to the higher mechanical strengths, lower water absorption, and lower thickness swelling achieved. Therefore, S-A-P lignin is a viable replacement for phenol to produce LPF resins that can be used for plywood and particleboard applications, which has potential for exterior use. 


\section{CONCLUSIONS AND RECOMMENDATIONS}

\subsection{Conclusions}

The overall aim of this study was achieved: phenol was replaced by technical lignin from South African spent pulping liquor, at up to $100 \%$ substitution to produce LPF resins for wood applications, while adhering to standard requirements. Soda lignin and sodium lignosulphonate were obtained, and they were isolated and purified in different ways. Thereafter, they were characterised to determine which lignin would be the most promising replacement for phenol to synthesise LPF resins.

Purified soda lignin was foreshadowed to be the most promising replacement for phenol, as it had a higher reactivity and fewer impurities compared to the lignosulphonate and impure soda lignins. From the compositional analysis, it was determined that S-A-P had the lowest ash content (2.16\%). This was favourable as a high ash content leads to poor reactivity, and a high degree of impurities could hinder the reaction with formaldehyde. S-A-P also had negligible nitrogen and sulphur present and the highest carbon and hydrogen contents, while the lignosulphonates had a significant sulphur content that remained from the sulphite pulping. The phenolic hydroxyl content was highest for S-A-P (1.70 mmol phenolic hydroxyls per gram sample) which foreshadowed a good reactivity with formaldehyde. From the mid-IR spectra, S-A-P had more H- and G units and fewer S-units compared to the other lignins, which foreshadowed a reduced steric hindrance and a higher reactivity. Furthermore, S-A-P was the most thermally stable with its maximum thermal degradation temperature at $425^{\circ} \mathrm{C}$.

Phenolation of the soda lignin cleaved weak bonds and increased the phenolic hydroxyl content up to $105 \%$ ( $3.48 \mathrm{mmol}$ phenolic hydroxyls per gram lignin) and 124\% (3.81 mmol phenolic hydroxyls per gram lignin) at $80 \%$ and $60 \%$ substitution, respectively. The optimum phenolation temperature was determined to be at 80 and $110{ }^{\circ} \mathrm{C}$ at $80 \%$ and $60 \%$ substitution, respectively. Phenolation at $60 \%$ and $80 \%$ substitution allowed the highest shear strengths to be reached at lower formaldehyde and sodium hydroxide additions required compared to other literature, which was attributed to phenolation enhancing the reactivity and hence lowering the activation energy required. Furthermore, after the phenolation of S-A-P, the mid-IR spectra showed that ether bonds were cleaved at $1265 \mathrm{~cm}^{-1}$ and the phenolic hydroxyl content increased at $1215 \mathrm{~cm}^{-1}$. A new peak at $754 \mathrm{~cm}^{-1}$ appeared after phenolation indicating linkages formed between the ortho- or parapositions on phenol and the $\alpha$-hydroxyl groups on the lignin side chain (Alonso et al., 2005). These new peaks indicated that phenol was successfully attached to the lignin units.

A one-pot phenolation and LPF synthesis method reported previously (Zhao et al., 2016), was used for the first time with a pulping lignin, which allowed the direct transition from the phenolation to LPF synthesis reactions in alkaline conditions, which could favour less chemical waste produced. The best synthesis reaction conditions from $60 \%$ to $80 \%$ substitution were at the lower bound of the range tested with a $\mathrm{NaOH} / \mathrm{PL}$ molar ratio of 0.124 and a F/PL molar ratio of 1 . This was lower than previously reported. At $100 \%$ substitution without any modification, the optimum $\mathrm{NaOH} / \mathrm{PL}$ molar ratio was at 0.477 , which corresponded to previous reports. The best F/PL molar ratio at $100 \%$ substitution in the tested range was at the upper bound at three. This was higher than expected based on previous reports. This high formaldehyde requirement was attributed to the high 
percentage incorporation of the less reactive soda lignin and no additives used, thus requiring a high formaldehyde content to overcome the higher activation energy and promote the forward synthesis reaction.

Shear strengths at $80 \%$ substitution performed better than at $60 \%$ and $100 \%$, which was governed by $80 \%$ substitution having a favourable degree of branched lignin units, while still having a high enough reactivity for the addition of formaldehyde and condensation to occur to form cross-linked networks. For these best performing resins at each substitution level, the $\mathrm{pH}$ and free formaldehyde contents adhered to that specified by industrial standards. The high shear strength performance obtained at all substitution levels at optimal reaction conditions tested was attributed to a high degree of cross-linking that occurred before the final curing reaction. This was confirmed by a high methylene bridge formation from the mid-IR data at $1470 \mathrm{~cm}^{-1}$, as well as a high thermal stability in the TGA results. The S-A-P LPF60 and LPF80 resins had their maximum thermal degradation peaks around $350{ }^{\circ} \mathrm{C}$, while it was around $250{ }^{\circ} \mathrm{C}$ for the LPF100 resins.

The good performance of this soda lignin as a LPF resin was further confirmed by particleboard produced with LPF68 giving a MOR of 23.5 MPa and a MOE of $2750 \mathrm{MPa}$, which adhered to the ANSI A208.1-1999 standard. Furthermore, good resistance to water absorption (37.2\%) and thickness swelling (13.5\%) was observed. This unclean pulping lignin is unique in its properties and was the first lignin to produce LPF resins at $100 \%$ substitution giving a shear strength of 0.786 without modification or additives required while adhering to plywood standard specifications. The highest shear strength of $1.11 \mathrm{MPa}$ was achieved at $68 \%$ substitution.

\subsection{Recommendations}

The sodium lignosulphonate used showed promise as a phenol replacement; however, further purifications and modifications need to be investigated to improve its properties for this application. Different purification methods could also be investigated to decrease the ash content of the lignosulphonates. Purification using a dialysis membrane with 3500 MWCO was done, but this only decreased the ash content from $24.8 \%$ to $12.8 \%$, which indicated that the impurities were attached to the lignin structure and dialysis would not able to remove it. The sulphur content was also only reduced from $7.5 \%$ to $5.6 \%$. Desulphonation methods may be investigated to reduce the sulphur content. Furthermore, lower molecular weight units of the lignin were also removed during dialysis, which was unfavourable for the LPF synthesis as the lower molecular weight units have a higher reactivity. Therefore, this trade-off between impurities and low molecular weight units could be further investigated. Different levels of substitution may be investigated, to determine what the maximum substitution level of this lignosulphonate is that may produce LPF resins with suitable properties for wood applications. Govender, et al. (2020) proved that hexamine as a cross-linker and epichlorohydrin as a hardener could improve the properties of the LPF100 resin from this L-S lignin to produce an acceptable shear strength. Another challenge of utilising lignosulphonate is its high hydrophilicity, which caused long drying times of the LPF resin after synthesis in the vacuum oven as well as occasional incomplete drying of the plywood during its conditioning stage, leading to delamination thereof. Hence, reducing the hydrophilicity could also be investigated.

For the S-A-P lignin, further investigation could be done to determine the economic feasibility of utilising it as a pulping by-product to produce LPF resins. The industrialisation of the acid precipitation and acid purification steps is unfavourable as it would require a lot of water and would lead to a lot of acid waste produced, which 
would have to be dealt with. Therefore, a different purification method or the disposal of the acid used could also be further investigated. The utilisation of crude black liquor as-is (without acid precipitation or purification) as a phenol substitute could also be investigated, as minimal processing steps would be favourable for industrial implementation. Furthermore, the best reaction conditions were determined to be at the upper or lower bounds in some cases; therefore, testing wider ranges is recommended. Further optimisation of the reaction parameters could be required for production on an industrial scale. Further investigation may also be done on the pressing process of the plywood and particleboards, where the effect of optimising the hot-press times and temperatures on the board properties could also be determined. 


\section{REFERENCES}

Abdelaziz, O.Y., Brink, D.P., Prothmann, J., Ravi, K., Sun, M., García-Hidalgo, J., Sandahl, M., Hulteberg, C.P., et al. 2016. Biological valorization of low molecular weight lignin. Biotechnology Advances. 34(8):1318-1346. DOI: 10.1016/j.biotechadv.2016.10.001.

Abdelwahab, N.A. \& Nassar, M.A. 2011. Preparation, optimisation and characterisation of lignin phenol formaldehyde resin as wood adhesive. Pigment and Resin Technology. 40(3):169-174. DOI: 10.1108/03699421111130432.

Alawode, A.O.O., Bungu, P.S.S.E.E., Amiandamhen, S.O.O., Meincken, M. \& Tyhoda, L. 2019. Properties and characteristics of novel formaldehyde-free wood adhesives prepared from Irvingia gabonensis and Irvingia wombolu seed kernel extracts. International Journal of Adhesion and Adhesives. 95:102423. DOI: 10.1016/j.ijadhadh.2019.102423.

Alonso, M. V., Rodrguez, J.J., Oliet, M., Rodrguez, F., Garca, J. \& Gilarranz, M.A. 2001. Characterization and structural modification of ammonic lignosulfonate by methylolation. Journal of Applied Polymer Science. 82(11):2661-2668. DOI: 10.1002/app.2119.

Alonso, M. V., Oliet, M., Rodríguez, F., Astarloa, G. \& Echeverría, J.M. 2004. Use of a methylolated softwood ammonium lignosulfonate as partial substitute of phenol in resol resins manufacture. Journal of Applied Polymer Science. 94(2):643-650. DOI: 10.1002/app.20887.

Alonso, M. V., Oliet, M., Rodríguez, F., García, J., Gilarranz, M.A. \& Rodríguez, J.J. 2005. Modification of ammonium lignosulfonate by phenolation for use in phenolic resins. Bioresource Technology. 96(9):10131018. DOI: 10.1016/j.biortech.2004.09.009.

Alonso, M. V., Oliet, M., Domínguez, J.C., Rojo, E. \& Rodríguez, F. 2011. Thermal degradation of lignin-phenolformaldehyde and phenol-formaldehyde resol resins: Structural changes, thermal stability, and kinetics. Journal of Thermal Analysis and Calorimetry. 105(1):349-356. DOI: 10.1007/s10973-011-1405-0.

Alonso Pippo, W., Luengo, C.A., Alonsoamador Morales Alberteris, L., Garzone, P. \& Cornacchia, G. 2011. Energy recovery from sugarcane-trash in the light of 2 nd generation biofuel. Part 2: Socio-economic aspects and techno-economic analysis. Waste and Biomass Valorization. 2(3):257-266. DOI: 10.1007/s12649-0119069-3.

Amen-Chen, C., Pakdel, H. \& Roy, C. 2001. Production of monomeric phenols by thermochemical conversion of biomass: A review. Bioresource Technology. 79(3):277-299. DOI: 10.1016/S0960-8524(00)00180-2.

Amiandamhen, S.O., Meincken, M. \& Tyhoda, L. 2018. The effect of chemical treatments of natural fibres on the properties of phosphate-bonded composite products. Wood Science and Technology. 52(3):653-675. DOI: 10.1007/s00226-018-0999-9.

Angelini, S., Barrio, A., Cerruti, P., Scarinzi, G., Garcia-Jaca, J., Savy, D., Piccolo, A. \& Malinconico, M. 2019. Lignosulfonates as fire retardants in wood flour-based particleboards. International Journal of Polymer Science. 2019. DOI: 10.1155/2019/6178163.

Areskogh, D., Li, J., Gellerstedt, G. \& Henriksson, G. 2010. Investigation of the Molecular Weight Increase of Commercial Lignosulfonates by Laccase Catalysis. Biomacromolecules. 11(4):904-910. DOI: 10.1021/bm901258v.

Aro, T. \& Fatehi, P. 2017. Production and Application of Lignosulfonates and Sulfonated Lignin. ChemSusChem. 10(9):1861-1877. DOI: $10.1002 / c s s c .201700082$.

Bajwa, D.S., Pourhashem, G., Ullah, A.H. \& Bajwa, S.G. 2019. A concise review of current lignin production, applications, products and their environment impact. Industrial Crops and Products. 139. DOI: 10.1016/j.indcrop.2019.111526.

Bekhta, P., Sedliacik, J. \& Bekhta, N. 2020. Effects of Selected Parameters on the Bonding Quality and Temperature Evolution Inside Plywood During Pressing. Polymers. 12(5):3-8. DOI: 
doi:10.3390/polym12051035.

Belgacem, M.N. \& Gandini, A. 2008. Monomers, Polymers and Composites from Renewable Resources. 1st ed. ed. M.N. Belgacem, A. Gandini, \& ScienceDirect (Online service), Eds. Amsterdam: Elsevier Ltd. DOI: 10.1016/B978-0-08-045316-3.X0001-4.

Bhattacharjee, G., Neogi, S. \& Das, S.K. 2014. Phenol-formaldehyde runaway reaction: a case study. International Journal of Industrial Chemistry. 5(2):1-6. DOI: 10.1007/s40090-014-0013-9.

Biermann, C.J. 1996. Handbook of pulping and papermaking. 2nd ed. ed. V. 34. San Diego: Academic Press. DOI: $10.5860 / C H O I C E .34-3322$.

Brebu, M. \& Vasile, C. 2010. Thermal degradation of lignin-A review. Cellulose chemistry and technology. 44(9):353-363.

Calace, N., Nardi, E., Petronio, B.M. \& Pietroletti, M. 2002. Adsorption of phenols by papermill sludges. Environmental Pollution. 118(3):315-319. DOI: 10.1016/S0269-7491(01)00303-7.

Çetin, N.S. \& Özmen, N. 2002. Use of organosolv lignin in phenol-formaldehyde resins for particleboard production. International Journal of Adhesion and Adhesives. 22(6):477-480. DOI: 10.1016/S01437496(02)00058-1.

Chen, H. 2014. Chemical Composition and Structure of Natural Lignocellulose. In Biotechnology of Lignocellulose. Springer Netherlands. 25-71. DOI: 10.1007/978-94-007-6898-7_2.

Chen, Y., Gong, X., Yang, G., Li, Q. \& Zhou, N. 2019. Preparation and characterization of a nanolignin phenol formaldehyde resin by replacing phenol partially with lignin nanoparticles. RSC Advances. 9(50):29255-29262. DOI: 10.1039/c9ra04827h.

Chen, Z., Chen, Y., Cai, H. \& Han, J. 2019. Processing variable optimization of plywood hot pressed with Ba2+modified phenol-formaldehyde resin by a response surface method. Forest Products Journal. 69(2):148-153. DOI: 10.13073/FPJ-D-18-00044.

Chio, C., Sain, M. \& Qin, W. 2019. Lignin utilization: A review of lignin depolymerization from various aspects. Renewable and Sustainable Energy Reviews. 107:232-249. DOI: 10.1016/j.rser.2019.03.008.

Collard, F.X. \& Blin, J. 2014. A review on pyrolysis of biomass constituents: Mechanisms and composition of the products obtained from the conversion of cellulose, hemicelluloses and lignin. Renewable and Sustainable Energy Reviews. 38:594-608. DOI: 10.1016/j.rser.2014.06.013.

Crawford, R.J. \& Throne, J.L. 2002. Rotational molding technology. J.L. Throne, Ed. (Plastics Design Library). Norwich, N.Y., N.Y.: Plastics Design Library/William Andrew Pub. DOI: 10.1016/B978-188420785-3.50005-8.

Demuner, I. F., Colodette, J. L., Demuner, A. J., and Jardim, C.M. 2019. Biorefinery review: Wide-reaching products through kraft lignin. BioRes. 14(3):7543-7581.

Dessbesell, L., Paleologou, M., Leitch, M., Pulkki, R. \& Xu, C. (Charles). 2020. Global lignin supply overview and kraft lignin potential as an alternative for petroleum-based polymers. Renewable and Sustainable Energy Reviews. 123(February):109768. DOI: 10.1016/j.rser.2020.109768.

Dhakal, H.N., Zhang, Z.Y. \& Richardson, M.O.W. 2007. Effect of water absorption on the mechanical properties of hemp fibre reinforced unsaturated polyester composites. Composites Science and Technology. 67(78):1674-1683. DOI: 10.1016/j.compscitech.2006.06.019.

Doherty, W.O.S., Mousavioun, P. \& Fellows, C.M. 2011. Value-adding to cellulosic ethanol: Lignin polymers. Industrial Crops and Products. 33(2):259-276. DOI: 10.1016/j.indcrop.2010.10.022.

Donmez Cavdar, A., Kalaycioglu, H. \& Hiziroglu, S. 2008. Some of the properties of oriented strandboard manufactured using kraft lignin phenolic resin. Journal of Materials Processing Technology. 202(1-3):559-563. DOI: 10.1016/j.jmatprotec.2007.10.039. 
Eckelman, B.C. a \& Eckelman, C.A. 1997. Brief Survey of Wood Adhesives. FNR 154. Purdue University Cooperative Extension: West Lafayette, IN. 10.

Ekielski, A. \& Mishra, P.K. 2021. Lignin for bioeconomy: The present and future role of technical lignin. International Journal of Molecular Sciences. 22(1):1-24. DOI: 10.3390/ijms22010063.

Eraghi Kazzaz, A., Hosseinpour Feizi, Z. \& Fatehi, P. 2019. Grafting strategies for hydroxy groups of lignin for producing materials. Green Chemistry. 21(21):5714-5752. DOI: 10.1039/c9gc02598g.

Fan, J. \& Zhan, H. 2008. Optimization of Synthesis of Spherical Lignosulphonate Resin and Its Structure Characterization. Chinese Journal of Chemical Engineering. 16(3):407-410. DOI: 10.1016/S10049541(08)60097-X.

Fatehi, P. \& Chen, J. 2016. Production of Biofuels and Chemicals from Lignin. Z. Fang \& R.L. Smith, Eds. (Biofuels and Biorefineries). Singapore: Springer Singapore. DOI: 10.1007/978-981-10-1965-4.

Fearon, O., Kuitunen, S. \& Vuorinen, T. 2016. Reaction kinetics of strong nucleophiles with a dimeric nonphenolic lignin model compound with $\alpha$-carbonyl functionality (adleron) in aqueous alkali solution. Holzforschung. 70(9):811-818. DOI: 10.1515/hf-2015-0236.

Ferdosian, F., Pan, Z., Gao, G. \& Zhao, B. 2017. Bio-based adhesives and evaluation for wood composites application. Polymers. 9(2). DOI: 10.3390/polym9020070.

Figoli, A., Alfredo, C. \& Basile, A. 2016. Part Two Integrated membrane operations for the recovery of chemical feedstocks. In Membrane Technologies for Biorefining. 96th ed. A. Figoli, A. Cassano, \& A. Basile, Eds. Woodhead Publishing. Available: https://app.knovel.com/hotlink/pdf/id:kt010W3EXM/membranetechnologies/raw-materials-pulp-production.

Gan, L. \& Pan, X. 2019. Phenol-Enhanced Depolymerization and Activation of Kraft Lignin in Alkaline Medium. Industrial and Engineering Chemistry Research. 58(19):7794-7800. DOI: 10.1021/acs.iecr.9b01147.

Gao, C., Li, M.M., Zhu, C., Hu, Y., Shen, T., Li, M.M., Ji, X., Lyu, G., et al. 2021. One-pot depolymerization, demethylation and phenolation of lignin catalyzed by $\mathrm{HBr}$ under microwave irradiation for phenolic foam preparation. Composites Part B: Engineering. 205(November 2020):108530. DOI: 10.1016/j.compositesb.2020.108530.

García, A., Toledano, A., Serrano, L., Egüés, I., González, M., Marín, F. \& Labidi, J. 2009. Characterization of lignins obtained by selective precipitation. Separation and Purification Technology. 68(2):193-198. DOI: 10.1016/j.seppur.2009.05.001.

Gellerstedt, G., Ek, M. \& Henriksson, G. 2009. Wood chemistry and biotechnology. Walter de Gruyter GmbH and Co. KG. DOI: 10.1515/9783110213409.

Ghorbani, M., Liebner, F., van Herwijnen, H.W.G., Pfungen, L., Krahofer, M., Budjav, E. \& Konnerth, J. 2016. Lignin phenol formaldehyde resoles: The impact of lignin type on adhesive properties. BioResources. 11(3):6727-6741. DOI: 10.15376/biores.11.3.6727-6741.

Ghorbani, M., Konnerth, J., van Herwijnen, H.W.G., Zinovyev, G., Budjav, E., Requejo Silva, A. \& Liebner, F. 2018. Commercial lignosulfonates from different sulfite processes as partial phenol replacement in PF resole resins. Journal of Applied Polymer Science. 135(8):1-11. DOI: 10.1002/app.45893.

Ghorbani, M., Mahendran, A.R., van Herwijnen, H.W.G., Liebner, F. \& Konnerth, J. 2018. Paper-based laminates produced with kraft lignin-rich phenol-formaldehyde resoles meet requirements for outdoor usage. European Journal of Wood and Wood Products. 76(2):481-487. DOI: 10.1007/s00107-017-1248-x.

Gierer, J. 1980. Chemical aspects of kraft pulping. Wood Sci.Technol. 14:241-266. DOI: https://doi.org/10.1007/BF00383453.

Glasser, W.G., Davé, V. \& Frazier, C.E. 1993. Molecular weight distribution of (semi-) commercial lignin derivatives. Journal of Wood Chemistry and Technology. 13(4):545-559. DOI: 10.1080/02773819308020533. 
Govender, P., Majeke, B.M., Alawode, A.O., Gorgens, J.F. \& Tyhoda, L. 2020. The use of south african spent pulping liquor to synthesize lignin phenol-formaldehyde resins. Forest Products Journal. 70(4):503-511. DOI: 10.13073/FPJ-D-20-00047.

Govender, P., Görgens, J. \& Tyhoda, L. 2020. Use of South African spent pulping liquor to synthesise lignin phenol formaldehyde resins. Stellenbosch University.

Gu, K., Huang, J. \& Li, K. 2013. Preparation and evaluation of particleboard bonded with a soy flour-based adhesive with a new curing agent. Journal of Adhesion Science and Technology. 27(18-19):2053-2064. DOI: 10.1080/01694243.2012.696950.

Hemmilä, V., Adamopoulos, S., Karlsson, O. \& Kumar, A. 2017. Development of sustainable bio-adhesives for engineered wood panels-A Review. RSC Advances. 7(61):38604-38630. DOI: 10.1039/c7ra06598a.

Henriksson, G., Li, J., Zhang, L. \& Lindström, Mikael, E. 2010. Lignin Utilization. In Thermochemical Conversion of Biomass to Liquid Fuels and Chemicals. Royal Society of Chemistry. 222-262. Available: https://app.knovel.com/hotlink/pdf/id:kt00AB3MY8/thermochemical-conversion/lignin-large-incompletely.

Hidayati, S., Satyajaya, W. \& Fudholi, A. 2020. Lignin isolation from black liquor from oil palm empty fruit bunch using acid. Journal of Materials Research and Technology. 9(5):11382-11391. DOI: 10.1016/j.jmrt.2020.08.023.

Howard, R.L., Abotsi, E., Jansen, van R.E.L. \& Howard, S. 2003. Lignocellulose biotechnology: issues of bioconversion and enzyme production. African Journal of Biotechnology. 2(12):602-619. DOI: 10.5897/AJB2003.000-1115.

Hu, L., Pan, H., Zhou, Y. \& Zhang, M. 2011. Methods to improve lignin's reactivity as a phenol substitute and as replacement for other phenolic compounds: A brief review. BioResources. 6(3):3515-3525. DOI: 10.15376/biores.6.3.3515-3525.

Hu, L., Zhou, Y., Zhang, M. \& Liu, R. 2012. Characteriztion and properties of a lignosulphonate-based phenolic foam. BioResources. 7(1):554-564.

Hu, L., Pan, H., Zhou, Y., Hse, C.Y., Liu, C., Zhang, B. \& Xu, B. 2014. Chemical groups and structural characterization of lignin via thiol-mediated demethylation. Journal of Wood Chemistry and Technology. 34(2):122-134. DOI: 10.1080/02773813.2013.844165.

Huang, J., Liu, W. \& Qiu, X. 2019. High Performance Thermoplastic Elastomers with Biomass Lignin as Plastic Phase. ACS Sustainable Chemistry and Engineering. 7(7):6550-6560. DOI: 10.1021/acssuschemeng.8b04936.

Hussin, M.H., Han Zhang, H., Aziz, N.A., Samad, N.A., Faris, A.H., Mohamad Ibrahim, M.N., Iqbal, A., Latip, A.F.A., et al. 2017. Preparation of environmental friendly phenol-formaldehyde wood adhesive modified with kenaf lignin. Beni-Suef University Journal of Basic and Applied Sciences. 6(4):409-418. DOI: 10.1016/j.bjbas.2017.06.004.

Iwakiri, V.T., Trianoski, R., Razera, D.L., Iwakiri, S. \& Rosa, T.S. da. 2019. Production of Structural Particleboard of Mimosa Scabrella Benth With Lignin Phenol-formaldehyde Resin. Floresta e Ambiente. 26(3). DOI: 10.1590/2179-8087.100617.

Jiang, X., Liu, J., Du, X., Hu, Z., Chang, H.M. \& Jameel, H. 2018. Phenolation to Improve Lignin Reactivity toward Thermosets Application. ACS Sustainable Chemistry and Engineering. 6(4):5504-5512. DOI: 10.1021/acssuschemeng.8b00369.

Jing, L., Jianlin, L., Qiang, G. \& Jianzhang, L. 2015. Effects of heat treatment on wet shear strength of plywood bonded with soybean meal-based adhesive. Industrial Crops and Products. 63:281-286. DOI: 10.1016/j.indcrop.2014.09.054.

Kalami, S., Arefmanesh, M., Master, E. \& Nejad, M. 2017. Replacing $100 \%$ of phenol in phenolic adhesive formulations with lignin. Journal of Applied Polymer Science. 134(30):45124. DOI: 10.1002/app.45124. 
Kalami, S., Chen, N., Borazjani, H. \& Nejad, M. 2018. Comparative analysis of different lignins as phenol replacement in phenolic adhesive formulations. Industrial Crops and Products. 125(August):520-528. DOI: 10.1016/j.indcrop.2018.09.037.

Karthäuser, J., Biziks, V., Mai, C. \& Militz, H. 2021. Lignin and Lignin-Derived Compounds for Wood Applications-A Review. Molecules. 26(9):2533. DOI: 10.3390/molecules26092533.

Karunarathna, M.S. \& Smith, R.C. 2020. Valorization of lignin as a sustainable component of structural materials and composites: Advances from 2011 to 2019. Sustainability (Switzerland). 12(2). DOI: $10.3390 /$ su12020734.

Kaur, R., Uppal, S.K. \& Sharma, P. 2017. Antioxidant and Antibacterial Activities of Sugarcane Bagasse Lignin and Chemically Modified Lignins. Sugar Tech. 19(6):675-680. DOI: 10.1007/s12355-017-0513-y.

Khan, M.A. \& Ashraf, S.M. 2007. Studies on thermal characterization of lignin: Substituted phenol formaldehyde resin as wood adhesives. Journal of Thermal Analysis and Calorimetry. 89(3):993-1000. DOI: 10.1007/s10973-004-6844-4.

Khan, M.A., Ashraf, S.M. \& Malhotra, V.P. 2004. Eucalyptus bark lignin substituted phenol formaldehyde adhesives: A study on optimization of reaction parameters and characterization. Journal of Applied Polymer Science. 92(6):3514-3523. DOI: 10.1002/app.20374.

Khokarale, S.G., Le-that, T. \& Mikkola, J. 2016. Carbohydrate Free Lignin: A Dissolution - Recovery Cycle of Sodium Lignosulfonate in a Switchable Ionic Liquid System. ACS Sustainable Chemistry \& Engineering. 4(12). DOI: 10.1021/acssuschemeng.6b01927.

Klapiszewski, L., Jamrozik, A., Strzemiecka, B., Matykiewicz, D., Voelkel, A. \& Jesionowski, T. 2017. Activation of magnesium lignosulfonate and kraft lignin: Influence on the properties of phenolic resin-based composites for potential applications in abrasive materials. International Journal of Molecular Sciences. 18(6):1-19. DOI: 10.3390/ijms18061224.

Kouisni, L., Fang, Y., Paleologou, M., Ahvazi, B., Hawari, J., Zhang, Y. \& Wang, X.M. 2011. Kraft lignin recovery and its use in the preparation of lignin-based phenol formaldehyde resins for plywood. Cellulose Chemistry and Technology. 45(7-8):515-520.

Laurichesse, S. \& Avérous, L. 2014. Chemical modification of lignins: Towards biobased polymers. Progress in Polymer Science. 39(7):1266-1290. DOI: 10.1016/j.progpolymsci.2013.11.004.

Lee, W., Chang, K.-C. \& Tseng, I.-M. (in press). Properties of phenol-formaldehyde resins prepared from phenol-liquefied lignin. Journal of Applied Polymer Science. 124(6):n/a-n/a. DOI: 10.1002/app.35539.

Lettner, M., Hesser, F., Hedeler, B., Schwarzbauer, P. \& Stern, T. 2020. Barriers and incentives for the use of lignin-based resins: Results of a comparative importance performance analysis. Journal of Cleaner Production. 256:120520. DOI: $10.1016 /$ j.jclepro.2020.120520.

Li, J.J., Wang, W., Zhang, S., Gao, Q., Zhang, W. \& Li, J.J. 2016. Preparation and characterization of lignin demethylated at atmospheric pressure and its application in fast curing biobased phenolic resins. RSC Advances. 6(71):67435-67443. DOI: 10.1039/c6ra11966b.

Li, J.J., Zhang, J., Zhang, S., Gao, Q., Li, J.J. \& Zhang, W. 2017. Fast curing bio-based phenolic resins via lignin demethylated under mild reaction condition. Polymers. 9(9). DOI: 10.3390/polym9090428.

Lin, L., Nakagame, S., Yao, Y., Yoshioka, M. \& Shiraishi, N. 2001. Liquefaction mechanism of $\beta$-O-4 lignin model compound in the presence of phenol under acid catalysis. Part 2. Reaction behavior and pathways. Holzforschung. 55(6):625-630. DOI: 10.1515/HF.2001.102.

Lin, L., Yao, Y. \& Shiraishi, N. 2001. Liquefaction mechanism of $\beta-O-4$ lignin model compound in the presence of phenol under acid catalysis. Part 1. Identification of the reaction products. Holzforschung. 55(6):617-624. DOI: 10.1515/HF.2001.101. 
Lora, J. 2008. Industrial Commercial Lignins: Sources, Properties and Applications. In Monomers, Polymers and Composites from Renewable Resources. Elsevier. 225-241. DOI: 10.1016/B978-0-08-045316-3.00010-7.

Lourençon, T. V., Alakurtti, S., Virtanen, T., Jääskeläïnen, A.S., Liitiä, T., Jaä\&die;skelaïnen, A.S., Liitiä, T., Hughes, M., et al. 2020. Phenol-formaldehyde resins with suitable bonding strength synthesized from "lessreactive" hardwood lignin fractions. Holzforschung. 74(2):175-183. DOI: 10.1515/hf-2018-0203.

Lu, F. \& Ralph, J. 2010. Lignin. In Cereal Straw as a Resource for Sustainable Biomaterials and Biofuels. 169207. DOI: 10.1016/B978-0-444-53234-3.00006-7.

Lu, Y., Lu, Y., Hu, H., Xie, F., Wei, X. \& Fan, X. 2017. Structural Characterization of Lignin and Its Degradation Products with Spectroscopic Methods. Journal of Spectroscopy. 2017:1-15. DOI: 10.1155/2017/8951658.

Ludmila, H., Michal, J., Andrea, Š. \& Aleš, H. 2015. Lignin, potential products and their market value. Wood Research. 60(6):973-986.

Ma, Y., Zhao, X., Chen, X. \& Wang, Z. 2011. An approach to improve the application of acid-insoluble lignin from rice hull in phenol-formaldehyde resin. Colloids and Surfaces A: Physicochemical and Engineering Aspects. 377(1-3):284-289. DOI: 10.1016/j.colsurfa.2011.01.006.

Macfarlane, A.L., Mai, M. \& Kadla, J.F. 2014. Bio-based chemicals from biorefining: Lignin conversion and utilisation. In Advances in Biorefineries: Biomass and Waste Supply Chain Exploitation. 659-692. DOI: 10.1533/9780857097385.2.659.

Madad, N., Chebil, L., Sanchez, C. \& Ghoul, M. 2011. Effect of molecular weight distribution on chemical, structural and physicochemical properties of sodium lignosulfonates. Rasayan Journal of Chemistry. 4(1):189202.

Malutan, T., Nicu, R. \& Popa, V.I. 2008. Contribution to the study of hydroxymethylation of alkali lignin. BioResources. 3(1):13-20.

El Mansouri, N.-E., Qiaolong, Y. \& Huang, F. 2018. Preparation and Characterization of Phenol-Formaldehyde Resins Modified with Alkaline Rice Straw Lignin. BioResources. 13(4):8061-8075. DOI: 10.15376/biores.13.4.8061-8075.

El Mansouri, N.E., Farriol, X. \& Salvadó, J. 2006. Structural modification and characterization of lignosulfonate by a reaction in an alkaline medium for its incorporation into phenolic resins. Journal of Applied Polymer Science. 102(4):3286-3292. DOI: 10.1002/app.24744.

Marques, A.P., Evtuguin, D. V., Magina, S., Amado, F.M.L. \& Prates, A. 2009. Chemical composition of Spent liquors from acidic magnesium-based Sulphite pulping of Eucalyptus globulus. Journal of Wood Chemistry and Technology. 29(4):322-336. DOI: 10.1080/02773810903207754.

Martínez, J.D. \& Velásquez, J.A. 2013. Effect of six technical lignins on thermo-mechanical properties of novolac type phenolic resins. Macromolecular Symposia. 333(1):197-205. DOI: 10.1002/masy. 201300057.

Marton, J., Marton, T., Falkehag, S.I. \& Adler, E. 1966. Alkali-Catalyzed Reactions of Formaldehyde with Lignins. In Lignin Structure and Reactions. 125-144. DOI: 10.1021/ba-1966-0059.ch010.

Maruthamuthu, M. 2017. Novel bacterial enzymes for plant biomass degradation discovered by meta-omics approach. Rijksuniversiteit Groningen. Available: http://www.rug.nl/research/portal. [2020, April 09].

Matsushita, Y. 2015. Conversion of technical lignins to functional materials with retained polymeric properties. Journal of Wood Science. 61(3):230-250. DOI: 10.1007/s10086-015-1470-2.

Mou, H., Li, B. \& Fardim, P. 2014. Pretreatment of corn stover with the modified hydrotropic method to enhance enzymatic hydrolysis. Energy and Fuels. 28(7):4288-4293. DOI: 10.1021/ef5001634.

Moubarik, A., Grimi, N., Boussetta, N. \& Pizzi, A. 2013. Isolation and characterization of lignin from Moroccan sugar cane bagasse: Production of lignin-phenol-formaldehyde wood adhesive. Industrial Crops and Products. 45:296-302. DOI: 10.1016/j.indcrop.2012.12.040. 
Nadji, H., Diouf, P.N., Benaboura, A., Bedard, Y., Riedl, B. \& Stevanovic, T. 2009. Comparative study of lignins isolated from Alfa grass (Stipa tenacissima L.). Bioresource Technology. 100(14):3585-3592. DOI: 10.1016/j.biortech.2009.01.074.

Namane, M., Sithole, B.B. \& Ramjugernath, D. 2015. Centrifugal washing and recovery as an improved method for obtaining lignin precipitated from South African kraft mill black liquor. Journal of Scientific and Industrial Research. 74:571-576.

Naron, D.R., Collard, F.X., Tyhoda, L. \& Görgens, J.F. 2017. Characterisation of lignins from different sources by appropriate analytical methods: Introducing thermogravimetric analysis-thermal desorption-gas chromatography-mass spectroscopy. Industrial Crops and Products. 101:61-74. DOI: 10.1016/j.indcrop.2017.02.041.

Nsaful, F., Collard, F.X., Carrier, M., Görgens, J.F. \& Knoetze, J.H. 2015. Lignocellulose pyrolysis with condensable volatiles quantification by thermogravimetric analysis - Thermal desorption/gas chromatography-mass spectrometry method. Journal of Analytical and Applied Pyrolysis. 116:86-95. DOI: 10.1016/j.jaap.2015.10.002.

Pang, B., Yang, S., Fang, W., Yuan, T.Q., Argyropoulos, D.S. \& Sun, R.C. 2017. Structure-property relationships for technical lignins for the production of lignin-phenol-formaldehyde resins. Industrial Crops and Products. 108:316-326. DOI: 10.1016/j.indcrop.2017.07.009.

Park, Y., Doherty, W.O.S. \& Halley, P.J. 2008. Developing lignin-based resin coatings and composites. Industrial Crops and Products. 27(2):163-167. DOI: 10.1016/j.indcrop.2007.07.021.

Parto, S.G., Christensen, J.M., Pedersen, L.S., Hansen, A.B., Tjosås, F., Spiga, C., Damsgaard, C.D., Larsen, D.B., et al. 2019. Liquefaction of Lignosulfonate in Supercritical Ethanol Using Alumina-Supported NiMo Catalyst. Energy \& Fuels. 33(2):1196-1209. DOI: 10.1021/acs.energyfuels.8b03519.

Pelaez-Samaniego, M.R., Yadama, V., Lowell, E. \& Espinoza-Herrera, R. 2013. A review of wood thermal pretreatments to improve wood composite properties. Wood Science and Technology. 47(6):1285-1319. DOI: 10.1007/s00226-013-0574-3.

Pfungen, L. 2015. Lignin Phenol Formaldehyde Wood Adhesives. University of Natural Resources and Life Sciences. Available: https://abstracts.boku.ac.at/download.php?dataset_id=12146\&property_id=107.

Pizzi, A. 2006. Recent developments in eco-efficient bio-based adhesives for wood bonding: opportunities and issues. Journal of Adhesion Science and Technology. 20(8):829-846. DOI: 10.1163/156856106777638635.

Pizzi, A. 2017. Handbook of Adhesive Technology, Third Edition. Third Edit ed. CRC Press. DOI: 10.1201/9781315120942.

Podschun, J., Saake, B. \& Lehnen, R. 2015. Reactivity enhancement of organosolv lignin by phenolation for improved bio-based thermosets. European Polymer Journal. 67:1-11. DOI: 10.1016/j.eurpolymj.2015.03.029.

Podschun, J., Stücker, A., Saake, B. \& Lehnen, R. 2015. Structure-function relationships in the phenolation of lignins from different sources. ACS Sustainable Chemistry and Engineering. 3(10):2526-2532. DOI: 10.1021/acssuschemeng.5b00705.

Podschun, J., Stü, A., Buchholz, R.I., Heitmann, M., Schreiber, A., Saake, B. \& Lehnen, R. 2016. Phenolated Lignins as Reactive Precursors in Wood Veneer and Particleboard Adhesion. Ind. Eng. Chem. Res. 55:25. DOI: 10.1021/acs.iecr.6b00594.

Ragnar, M., Lindgren, C.T. \& Nilvebrant, N.O. 2000. pKa-values of guaiacyl and syringyl phenols related to lignin. Journal of Wood Chemistry and Technology. 20(3):277-305. DOI: 10.1080/02773810009349637.

Renedo, M.J. \& Fernández-Ferreras, J. 2016. Characterization and Behavior of Modified Calcium-HydroxideBased Sorbents in a Dry Desulfurization Process. Energy \& Fuels. 30(8):6350-6354. DOI: 10.1021/acs.energyfuels.6b01106. 
Rezanowich, A., Yean, W.Q. \& Goring, D.A.I. 1964. High resolution electron microscopy of sodium lignin sulfonate. Journal of Applied Polymer Science. 8(4):1801-1812. DOI: 10.1002/app.1964.070080429.

Ringena, O., Saake, B. \& Lehnen, R. 2005. Isolation and fractionation of lignosulfonates by amine extraction and ultrafiltration: A comparative study. Holzforschung. 2(4):59-63. DOI: 10.1515/HF.2005.066.

Rogošić, M., Mencer, H.J. \& Gomzi, Z. 1996. Polydispersity index and molecular weight distributions of polymers. European Polymer Journal. 32(11):1337-1344. DOI: 10.1016/S0014-3057(96)00091-2.

Rowell, R.M. 2005. Handbook of Wood Chemistry and Wood Composites. 2nd ed. ed. Boca Raton, FL: CRC Press. DOI: 10.1201/9780203492437.

Ruwoldt, J. 2020. A Critical Review of the Physicochemical Properties of Lignosulfonates: Chemical Structure and Behavior in Aqueous Solution, at Surfaces and Interfaces. Surfaces. 3(4):622-648. DOI: 10.3390/surfaces3040042.

Sameni, J., Krigstin, S., Rosa, D. dos S., Leao, A. \& Sain, M. 2014. Thermal characteristics of lignin residue from industrial processes. BioResources. 9(1):725-737. DOI: 10.15376/biores.9.1.725-737.

Sarkar, S. \& Adhikari, B. 2000. Lignin-modified phenolic resin: Synthesis optimization, adhesive strength, and thermal stability. Journal of Adhesion Science and Technology. 14(9):1179-1193. DOI: 10.1163/156856100743167.

Sawamura, K., Tobimatsu, Y., Kamitakahara, H. \& Takano, T. 2017. Lignin Functionalization through Chemical Demethylation: Preparation and Tannin-Like Properties of Demethylated Guaiacyl-Type Synthetic Lignins. ACS Sustainable Chemistry and Engineering. 5(6):5424-5431. DOI: 10.1021/acssuschemeng.7b00748.

Shao, L., Zhang, X., Chen, F. \& Xu, F. 2017. Fast pyrolysis of Kraft lignins fractionated by ultrafiltration. Journal of Analytical and Applied Pyrolysis. 128:27-34. DOI: 10.1016/j.jaap.2017.11.003.

Shrivastava, A. 2018. Polymerization. In Introduction to Plastics Engineering. Elsevier. 17-48. DOI: 10.1016/B978-0-323-39500-7.00002-2.

Siddiqui, H., Mahmood, N., Yuan, Z., Crapulli, F., Dessbesell, L., Rizkalla, A., Ray, A. \& Xu, C. 2017. Sustainable bio-based phenol-formaldehyde resoles using hydrolytically depolymerized kraft lignin. Molecules. 22(11):119. DOI: $10.3390 /$ molecules 22111850 .

Sixta, H. Ed. 2006. Handbook of Pulp. V. 1-2. Weinheim: Wiley. DOI: 10.1002/9783527619887.

Solt, P., van Herwijnen, H.W.G. \& Konnerth, J. 2019. Thermoplastic and moisture-dependent behavior of lignin phenol formaldehyde resins. Journal of Applied Polymer Science. 136(40):48011. DOI: 10.1002/app.48011.

Solt, P., Jääskeläinen, A.S., Lingenfelter, P., Konnerth, J. \& Van Herwijnen, H.W.G. 2019. Impact of molecular weight of kraft lignin on adhesive performance of lignin-based phenol-formaldehyde resins. Forest Products Journal. 68(4):365-371. DOI: 10.13073/FPJ-D-17-00079.

Song, Y., Wang, Z., Yan, N., Zhang, R. \& Li, J. 2016. Demethylation of wheat straw alkali lignin for application in phenol formaldehyde adhesives. Polymers. 8(6). DOI: 10.3390/polym8060209.

Stephanou, A. \& Pizzi, A. 1993. Rapid-curing lignin-based exterior wood adhesives: Part II: Esters acceleration mechanism and application to panel products. Holzforschung. 47(6):501-506. DOI: 10.1515/hfsg.1993.47.6.501.

Stücker, A., Schütt, F., Saake, B. \& Lehnen, R. 2016. Lignins from enzymatic hydrolysis and alkaline extraction of steam refined poplar wood: Utilization in lignin-phenol-formaldehyde resins. Industrial Crops and Products. 85:300-308. DOI: 10.1016/j.indcrop.2016.02.062.

Tachon, N., Benjelloun-Mlayah, B. \& Delmas, M. 2016. Organosolv wheat straw lignin as a phenol substitute for green phenolic resins. BioResources. 11(3):5797-5815. DOI: 10.15376/biores.11.3.5797-5815.

Taleb, F., Ammar, M., Mosbah, M. ben, Salem, R. ben \& Moussaoui, Y. 2020. Chemical modification of lignin 
derived from spent coffee grounds for methylene blue adsorption. Scientific Reports. 10(1):1-13. DOI: 10.1038/s41598-020-68047-6.

Taverna, M.E., Felissia, F., Area, M.C., Estenoz, D.A. \& Nicolau, V.V. 2019. Hydroxymethylation of technical lignins from South American sources with potential use in phenolic resins. Journal of Applied Polymer Science. 136(26):47712. DOI: 10.1002/app.47712.

Tejado, A., Peña, C., Labidi, J., Echeverria, J.M. \& Mondragon, I. 2007. Physico-chemical characterization of lignins from different sources for use in phenol-formaldehyde resin synthesis. Bioresource Technology. 98(8):1655-1663. DOI: 10.1016/j.biortech.2006.05.042.

Thébault, M., Kutuzova, L., Jury, S., Eicher, I., Zikulnig-Rusch, E.M. \& Kandelbauer, A. 2020. Effect of phenolation, lignin-type and degree of substitution on the properties of lignin-modified phenol-formaldehyde impregnation resins: Molecular weight distribution, wetting behavior, rheological properties and thermal curing profiles. Journal of Renewable Materials. 8(6):603-630. DOI: 10.32604/jrm.2020.09616.

Tian, X., Fang, Z., Smith, R.L., Wu, Z. \& Liu, M. 2016. Properties, Chemical Characteristics and Application of Lignin and Its Derivatives. Springer, Singapore. 3-33. DOI: 10.1007/978-981-10-1965-4_1.

Tolbert, A., Akinosho, H., Khunsupat, R., Naskar, A.K. \& Ragauskas, A.J. 2014. Characterization and analysis of the molecular weight of lignin for biorefining studies. Biofuels, Bioproducts and Biorefining. 8(6):836-856. DOI: 10.1002/bbb.1500.

Tonge, L. 2007. Thermochemical Properties of Methylol Phenol Monomers and Phenol Formaldehyde Resoles. Swinburne University of Technology. DOI: 10.1016/B978-0-7234-5558-5.00001-4.

Vanholme, R., Demedts, B., Morreel, K., Ralph, J. \& Boerjan, W. 2010. Lignin biosynthesis and structure. Plant Physiology. 153(3):895-905. DOI: 10.1104/pp.110.155119.

Venkatesagowda, B. \& Dekker, R.F.H. 2019. Enzymatic demethylation of Kraft lignin for lignin-based phenolformaldehyde resin applications. Biomass Conversion and Biorefinery. DOI: 10.1007/s13399-019-00407-3.

Vishtal, A. \& Kraslawski, A. 2011. Challenges in industrial applications of technical lignins. BioResources. 6(3):3547-3568. DOI: 10.15376/biores.6.3.3547-3568.

Vural, D., Smith, J.C. \& Petridis, L. 2018. Dynamics of the lignin glass transition. Physical Chemistry Chemical Physics. 20(31):20504-20512. DOI: 10.1039/c8cp03144d.

Wang, C. \& Venditti, R.A. 2015. UV Cross-Linkable Lignin Thermoplastic Graft Copolymers. ACS Sustainable Chemistry and Engineering. 3(8):1839-1845. DOI: 10.1021/acssuschemeng.5b00416.

Wang, G. \& Chen, H. 2014. Carbohydrate elimination of alkaline-extracted lignin liquor by steam explosion and its methylolation for substitution of phenolic adhesive. Industrial Crops and Products. 53:93-101. DOI: 10.1016/j.indcrop.2013.12.020.

Wang, H., Eberhardt, T.L., Wang, C., Gao, S. \& Pan, H. 2019. Demethylation of alkali lignin with halogen acids and its application to phenolic resins. Polymers. 11(11):1-16. DOI: 10.3390/polym11111771.

Wang, M., Zhao, Y. \& Li, J. 2018. Demethylation and other modifications of industrial softwood kraft lignin by laccase-mediators. Holzforschung. 72(5):357-365. DOI: 10.1515/hf-2017-0096.

Wool, R. \& Sun, X.S. 2005. Lignin Polymers and Composites. In Bio-Based Polymers and Composites. Elsevier. 551-598. DOI: 10.1016/b978-012763952-9/50017-4.

Wu, S. \& Zhan, H. 2001. Characteristics of demethylated wheat straw soda lignin and its utilization in ligninbased phenolic formaldehyde resins. Cellulose Chemistry and Technology. 35(3-4):253-262. Available: https://pascal-francis.inist.fr/vibad/index.php?action=getRecordDetail\&idt=14620522 [2020, June 04].

Xian, C.K., Othman, N., Harruddin, N., Nasruddin, N.A. \& Zing, Y.O. 2014. Extraction of Lignosulfonate using TOA-Kerosene-PVDF in Supported Liquid Membrane Process. Jurnal Teknologi. 2:59-63. 
Xu, Y., Guo, L., Zhang, H., Zhai, H. \& Ren, H. 2019. Research status, industrial application demand and prospects of phenolic resin. RSC Advances. 9(50):28924-28935. DOI: 10.1039/c9ra06487g.

Yaakob, M.N.A., Bin Roslan, R., Salim, N. \& Zakaria, S. 2021. Comparison of Phenol-Formaldehyde and LigninFormaldehyde Resin Adhesives for Wood Application. Materials Science Forum. 1025:307-311. DOI: 10.4028/www.scientific.net/msf.1025.307.

Yan, M. \& Yang, D. 2015. Adsorption mechanism of lignosulfonate at the air/liquid interface. Journal of the Brazilian Chemical Society. 26(3):555-561. DOI: 10.5935/0103-5053.20150010.

Yang, S., Wen, J.-L., Yuan, T.-Q. \& Sun, R.-C. 2014. Characterization and phenolation of biorefinery technical lignins for lignin-phenol-formaldehyde resin adhesive synthesis. RSC Adv. 4(101):57996-58004. DOI: 10.1039/C4RA09595B.

Yang, S., Zhang, Y., Yuan, T.Q. \& Sun, R.C. 2015. Lignin-phenol-formaldehyde resin adhesives prepared with biorefinery technical lignins. Journal of Applied Polymer Science. 132(36):1-8. DOI: 10.1002/app.42493.

Yang, W., Rallini, M., Natali, M., Kenny, J., Ma, P., Dong, W., Torre, L. \& Puglia, D. 2019. Preparation and properties of adhesives based on phenolic resin containing lignin micro and nanoparticles: A comparative study. Materials and Design. 161:55-63. DOI: 10.1016/j.matdes.2018.11.032.

Zhang, Z.C. 2013. Catalytic transformation of carbohydrates and lignin in ionic liquids. Wiley Interdisciplinary Reviews: Energy and Environment. 2(6):655-672. DOI: 10.1002/wene.67.

Zhang, H., Fu, S. \& Chen, Y. 2020. Basic understanding of the color distinction of lignin and the proper selection of lignin in color-depended utilizations. International Journal of Biological Macromolecules. 147:607-615. DOI: 10.1016/j.ijbiomac.2020.01.105.

Zhang, W., Ma, Y., Wang, C., Li, S., Zhang, M. \& Chu, F. 2013. Preparation and properties of lignin-phenolformaldehyde resins based on different biorefinery residues of agricultural biomass. Industrial Crops and Products. 43(1):326-333. DOI: 10.1016/j.indcrop.2012.07.037.

Zhao, L.W., Griggs, B.F., Chen, C.L., Gratzl, J.S. \& Hse, C.Y. 1994. Utilization of softwood kraft lignin as adhesive for the manufacture of reconstituted wood. Journal of Wood Chemistry and Technology. 14(1):127-145. DOI: 10.1080/02773819408003090.

Zhao, M., Jing, J., Zhu, Y., Yang, X., Wang, X. \& Wang, Z. 2016. Preparation and performance of lignin-phenolformaldehyde adhesives. International Journal of Adhesion and Adhesives. 64:163-167. DOI: 10.1016/j.ijadhadh.2015.10.010.

Zhou, H., Zhu, J.Y., Gleisner, R., Qiu, X., Horn, E. \& Negrón, J. 2016. Pilot-scale demonstration of SPORL for bioconversion of lodgepole pine to bioethanol and lignosulfonate. Holzforschung. 70(1):21-30. DOI: 10.1515/hf-2014-0332.

Zhou, M., Shi, H., Li, C., Sheng, X., Sun, Y., Hou, M., Niu, M. \& Pan, X. 2020. Depolymerization and Activation of Alkali Lignin by Solid Acid-Catalyzed Phenolation for Preparation of Lignin-Based Phenolic Foams. Industrial \& Engineering Chemistry Research. DOI: 10.1021/acs.iecr.0c01753. 


\section{ADDENDUM A - COMPARISON OF DIFFERENT PHENOLATION AND SYNTHESIS REACTION CONDITIONS FROM PREVIOUS REPORTS}

Table 20. Different reaction parameters of phenolation in alkaline conditions as used in previous reports.

\begin{tabular}{|c|c|c|c|c|c|}
\hline & (Hu et al., 2012) & (Yang et al., 2014) & (Zhao et al., 2016) & (Ghorbani et al., 2018) & (Gan \& Pan, 2019) \\
\hline Lignin type & Lignosulphonate & Steam explosion/Kraft & Steam explosion & Lignosulphonates & Kraft \\
\hline Substitution \% & 14.3 & 50 & $20-70$ & 14.3 & 25 \\
\hline $\mathrm{NaOH}$ concentration & pH 9-10 & pH 9-10 & 8 wt.\% & pH 9-10 & 5 wt. $\%$ \\
\hline Temperature $\left({ }^{\circ} \mathrm{C}\right)$ & $100-120$ & 95 & 90 & 120 & 160 \\
\hline Time (hours) & 1 & 1 & 1.5 & 2 & 2 \\
\hline
\end{tabular}

Table 21. Different reaction parameters of phenolation in acidic conditions as used in previous reports.

\begin{tabular}{|c|c|c|c|c|c|}
\hline & (Ma et al., 2011) & (Lee et al., in press) & (Podschun et al., 2016) & (Jiang et al., 2018) & (Thébault et al., 2020) \\
\hline Type & Acid insoluble rice husks & Lignosulphonate & Beech organosolv & Kraft/Enzymatic hydrolysis & Lignosulphonate/Kraft \\
\hline Substitution \% & 14.3 & 28.6 & 33 & $28.5-37.5$ & 54 \\
\hline $\begin{array}{l}\mathrm{H}_{2} \mathrm{SO}_{4} \\
\text { concentration }\end{array}$ & $1.5 \mathrm{vol} \%$ & 5 wt.\% & 6.3 wt. $\%$ & 5 wt.\% & $1.6 w t \%$ \\
\hline Temperature $\left({ }^{\circ} \mathrm{C}\right)$ & 80 & 130 & 110 & $90-110$ & $75-100$ \\
\hline Time (hours) & 4 & 1 & 0.333 & 2 & $1-3$ \\
\hline
\end{tabular}


Table 22. Different LPF synthesis parameters as used in previous reports.

\begin{tabular}{|c|c|c|c|c|c|c|c|c|c|c|c|c|}
\hline Reference & Lignin type & $\begin{array}{l}\text { Substitution } \\
\text { (wt\%) }\end{array}$ & $\begin{array}{l}\text { F/P } \\
\text { molar } \\
\text { ratio }\end{array}$ & $\begin{array}{c}\mathrm{NaOH} / \mathrm{P} \\
\text { molar ratio }\end{array}$ & $\begin{array}{l}\text { Addition } \\
\mathrm{T}\left({ }^{\circ} \mathrm{C}\right)\end{array}$ & $\begin{array}{c}\text { Resole } \\
\text { pH } \\
\text { value }\end{array}$ & $\begin{array}{l}\text { Conden } \\
\text { sation t } \\
\text { (h) }\end{array}$ & $\begin{array}{c}\text { Conden } \\
\text { sation } \mathrm{T} \\
\left({ }^{\circ} \mathrm{C}\right)\end{array}$ & $\begin{array}{c}\text { Pressing } \\
\text { time } \\
\text { (min) }\end{array}$ & $\begin{array}{c}\text { Pressing } \\
\mathrm{T}\left({ }^{\circ} \mathrm{C}\right)\end{array}$ & $\begin{array}{c}\text { Curing } \\
\text { P } \\
(\mathrm{MPa})\end{array}$ & $\begin{array}{c}\text { Spread } \\
\text { rate } \\
\left(\mathrm{g} / \mathrm{m}^{2}\right)\end{array}$ \\
\hline $\begin{array}{l}\text { (Kalami et } \\
\text { al., 2018) }\end{array}$ & $\begin{array}{l}\text { Various } \\
\text { pulping } \\
\text { lignins }\end{array}$ & $100 \%$ & 2 & & 65 & $9-11$ & 2 & 90 & $3-4$ & 180 & 1.4 & \\
\hline $\begin{array}{l}\text { (Yang et al., } \\
\text { 2015) }\end{array}$ & $\begin{array}{l}\text { Biorefinery } \\
\text { lignins }\end{array}$ & $50 \%$ & 1.8 & & & $9-10$ & 2 & $80-90$ & 1.5 & 145 & 1 & 150 \\
\hline $\begin{array}{l}\text { (Zhang et } \\
\text { al., 2013) }\end{array}$ & $\begin{array}{l}\text { Agricultural } \\
\text { biorefinery }\end{array}$ & $30 \%-50 \%$ & 2 & 0.2 & & $9-12$ & 1 & 80 & 7 & 135 & 1.2 & $125-150$ \\
\hline $\begin{array}{l}\text { (Sarkar \& } \\
\text { Adhikari, } \\
\text { 2000) }\end{array}$ & Black liquor & $20 \%-50 \%$ & $\begin{array}{c}1.7 \\
(1.7-3.5)\end{array}$ & $\begin{array}{c}\mathbf{0 . 2 2} \\
(0.15-0.95)\end{array}$ & $25,65,80$ & $9.5-10.5$ & 3 & $92-95$ & & & & \\
\hline
\end{tabular}

*Values in bold were found to be optimums. 
Table 22. Different LPF synthesis parameters as used in previous reports (Continued)

\begin{tabular}{|c|c|c|c|c|c|c|c|c|c|c|c|c|}
\hline Reference & Lignin type & $\begin{array}{l}\text { Substitution } \\
\text { [mass \%] }\end{array}$ & $\begin{array}{c}\mathrm{F} / \mathrm{P} \\
\text { molar } \\
\text { ratio }\end{array}$ & $\begin{array}{c}\mathrm{NaOH} / \mathrm{P} \\
\text { molar ratio }\end{array}$ & $\begin{array}{l}\text { Addition } \\
\mathrm{T}\left[{ }^{\circ} \mathrm{C}\right]\end{array}$ & $\begin{array}{c}\text { Resole } \\
\mathrm{pH} \\
\text { value }\end{array}$ & $\begin{array}{c}\text { Conden } \\
\text { sation t } \\
\text { [h] }\end{array}$ & $\begin{array}{c}\text { Conden } \\
\text { sation } \mathrm{T} \\
{\left[{ }^{\circ} \mathrm{C}\right]}\end{array}$ & $\begin{array}{l}\text { Pressing t } \\
\text { [min] }\end{array}$ & $\begin{array}{c}\text { Pressing } \\
\mathrm{T}\left[{ }^{\circ} \mathrm{C}\right]\end{array}$ & $\begin{array}{c}\text { Curing } \\
\mathbf{P} \\
{[\mathrm{MPa}]}\end{array}$ & $\begin{array}{c}\text { Spread } \\
\text { rate } \\
{\left[\mathrm{g} / \mathrm{m}^{2}\right]}\end{array}$ \\
\hline $\begin{array}{l}\text { (Siddiqui et } \\
\text { al., 2017) }\end{array}$ & $\begin{array}{l}\text { Softwood } \\
\text { Kraft lignin }\end{array}$ & $\begin{array}{c}\mathbf{5 0 \%} \\
(0 \%-75 \%)\end{array}$ & $\begin{array}{c}1.2,2.1 \\
\mathbf{3}\end{array}$ & & 60 & & 2 & 80 & & & & \\
\hline $\begin{array}{l}\text { (Moubarik } \\
\text { et al., } \\
2013 \text { ) }\end{array}$ & $\begin{array}{l}\text { Sugarcane } \\
\text { bagasse }\end{array}$ & $30 \%$ & 2.2 & & & & & 90 & 6 & 160 & 1.2 & 225 \\
\hline $\begin{array}{l}\text { (Govender, } \\
\text { Görgens, et } \\
\text { al., 2020) }\end{array}$ & $\begin{array}{l}\text { Kraft, soda, } \\
\text { lignosulpho } \\
\text { nate }\end{array}$ & $100 \%$ & 2.5 & 0.3 & 65 & & 1 & 85 & 10 & 150 & & 350 \\
\hline $\begin{array}{l}\text { (Yaakob et } \\
\text { al., 2021) }\end{array}$ & $\begin{array}{l}\text { Acetosolv } \\
\text { lignin }\end{array}$ & $100 \%$ & 2.2 & & 65 & & 4 & 85 & & & & \\
\hline
\end{tabular}

*Values in bold were found to be optimums. 


\section{ADDENDUM B - SHEAR STRENGTH RESULTS OF THE LPF RESINS}

Table 23. Shear strength results from the CCD runs with the S-A-P LPF100, LPF80 and LPF60 resins.

\begin{tabular}{ccc}
\hline $\begin{array}{c}\text { NaOH } \\
\text { (weight fraction) }\end{array}$ & $\begin{array}{c}\text { F/PL } \\
\text { (molar ratio) }\end{array}$ & $\begin{array}{c}\text { Shear strength (MPa) } \\
\text { S-A-P LPF100 }\end{array}$ \\
\hline 0.05 & 1 & 0.385 \\
0.05 & 3 & 0.470 \\
0.22 & 1 & 0.172 \\
0.22 & 3 & 0.469 \\
0.05 & 2 & 0.084 \\
0.135 & 3 & 0.661 \\
0.22 & 2 & 0.786 \\
0.135 & 1 & 0.693 \\
0.135 & 2 & 0.452 \\
0.135 & 2 & 0.443 \\
0.135 & 2 & 0.386 \\
0.135 & 2 & 0.438 \\
\hline \multicolumn{2}{c}{ Average of centre points } \\
Standard deviation of centre points & 0.430 \\
\hline
\end{tabular}

\begin{tabular}{ccccc}
\hline $\begin{array}{c}\text { NaOH } \\
\text { (weight fraction) }\end{array}$ & $\begin{array}{c}\text { F/PL } \\
\text { (molar ratio) }\end{array}$ & $\begin{array}{c}\text { Temperature } \\
\left({ }^{\circ} \mathbf{C}\right)\end{array}$ & \multicolumn{2}{c}{ Shear strength (MPa) } \\
\hline 0.05 & 1 & 80 & 0.930 & 0.544 \\
0.05 & 3 & 80 & 0.965 & 0.289 \\
0.05 & 3 & 110 & 0.412 & 0.647 \\
0.05 & 1 & 110 & 0.916 & 0.851 \\
0.22 & 1 & 80 & 1.089 & 0.522 \\
0.22 & 3 & 80 & 1.020 & 0.457 \\
0.22 & 3 & 110 & 0.272 & 0.333 \\
0.22 & 1 & 110 & 0.369 & 0.830 \\
0.05 & 2 & 95 & 0.822 & 0.802 \\
0.135 & 3 & 95 & 0.444 & 0.107 \\
0.22 & 2 & 95 & 0.855 & 0.423 \\
0.135 & 1 & 95 & 0.887 & 0.987 \\
0.135 & 2 & 80 & 0.633 & 0.349 \\
0.135 & 2 & 110 & 0.576 & 0.575 \\
0.135 & 2 & 95 & 0.511 & 0.411 \\
0.135 & 2 & 95 & 0.457 & 0.436 \\
0.135 & 2 & 95 & 0.608 & 0.444 \\
0.135 & 2 & 95 & 0.462 & 0.345 \\
0.135 & 2 & 95 & 0.609 & \\
\hline & Average of centre points & 0.529 & 0.409 \\
& & & 0.067 & 0.039 \\
\hline
\end{tabular}




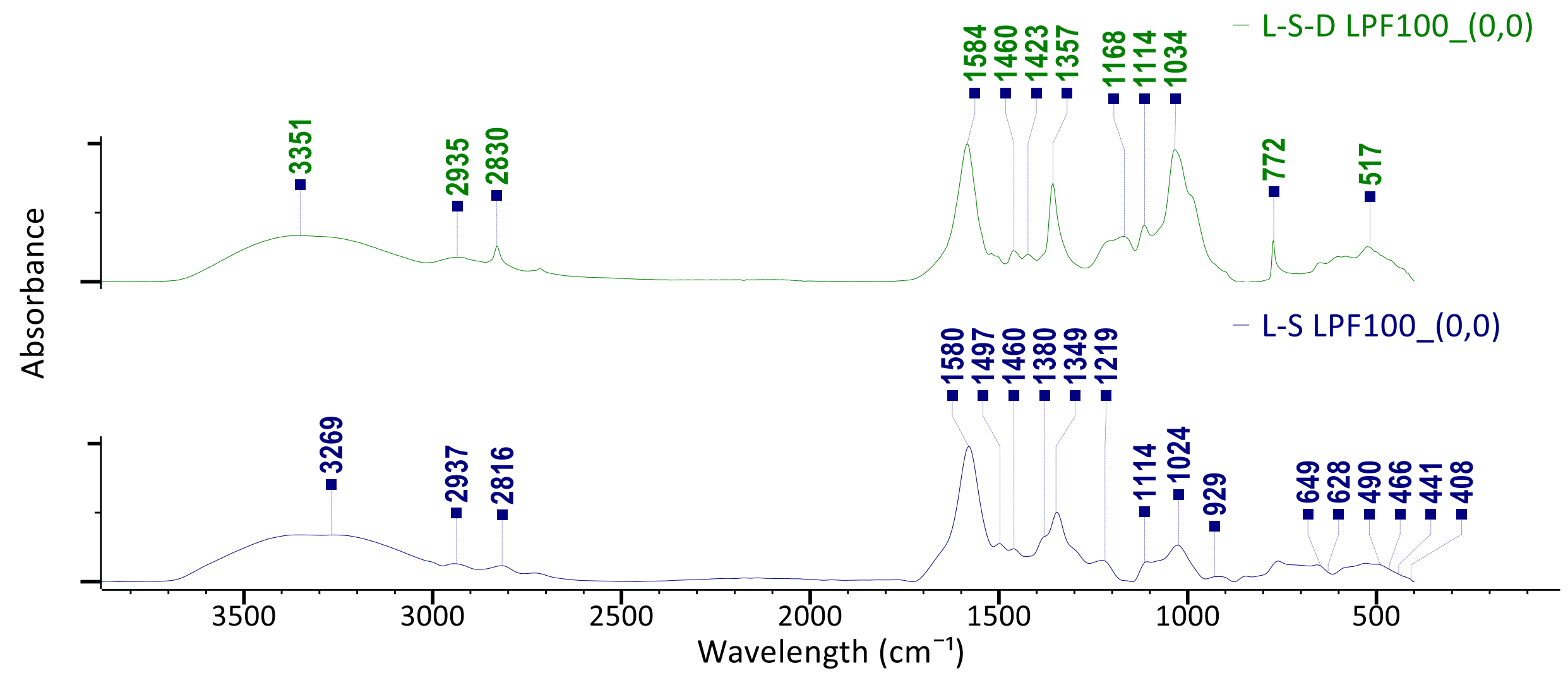

Figure 29. Mid-IR spectra of the LPF100 lignosulphonate resins at centre point conditions. 


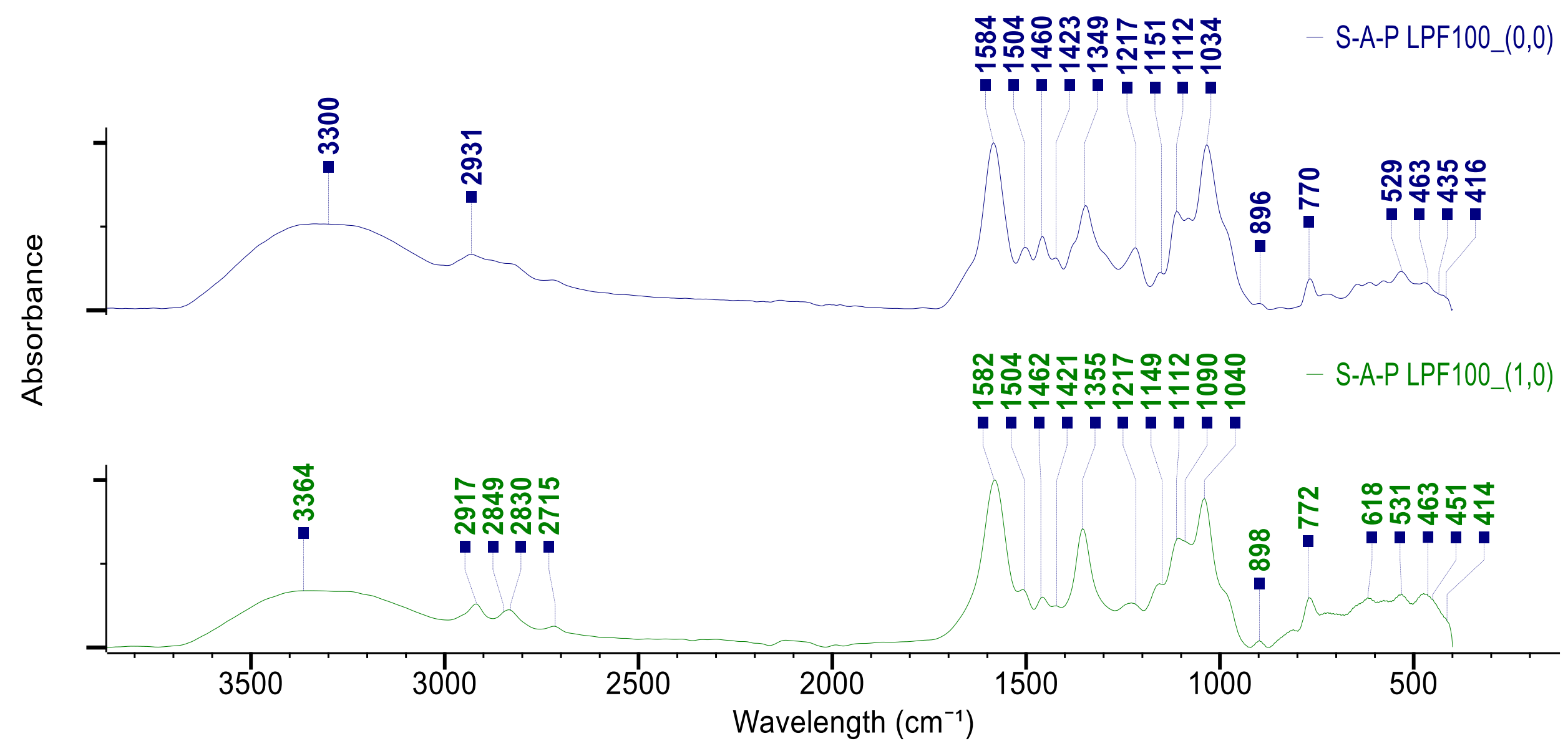

Figure 30. Mid-IR spectra of the S-A-P LPF100 resins of the centre point and highest shear samples. 


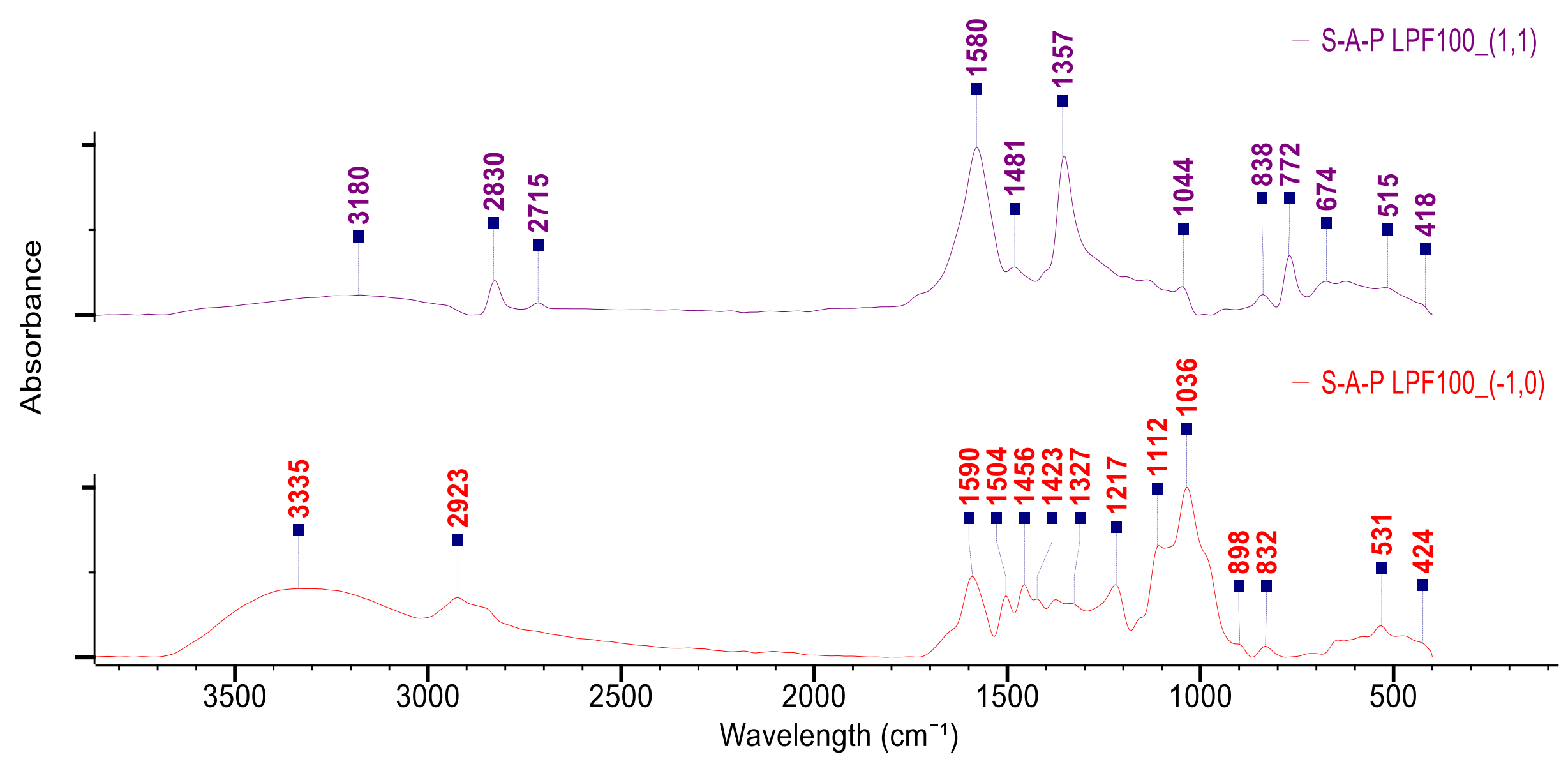

Figure 31. Mid-IR spectra of the S-A-P LPF100 resins with low shear strengths. 


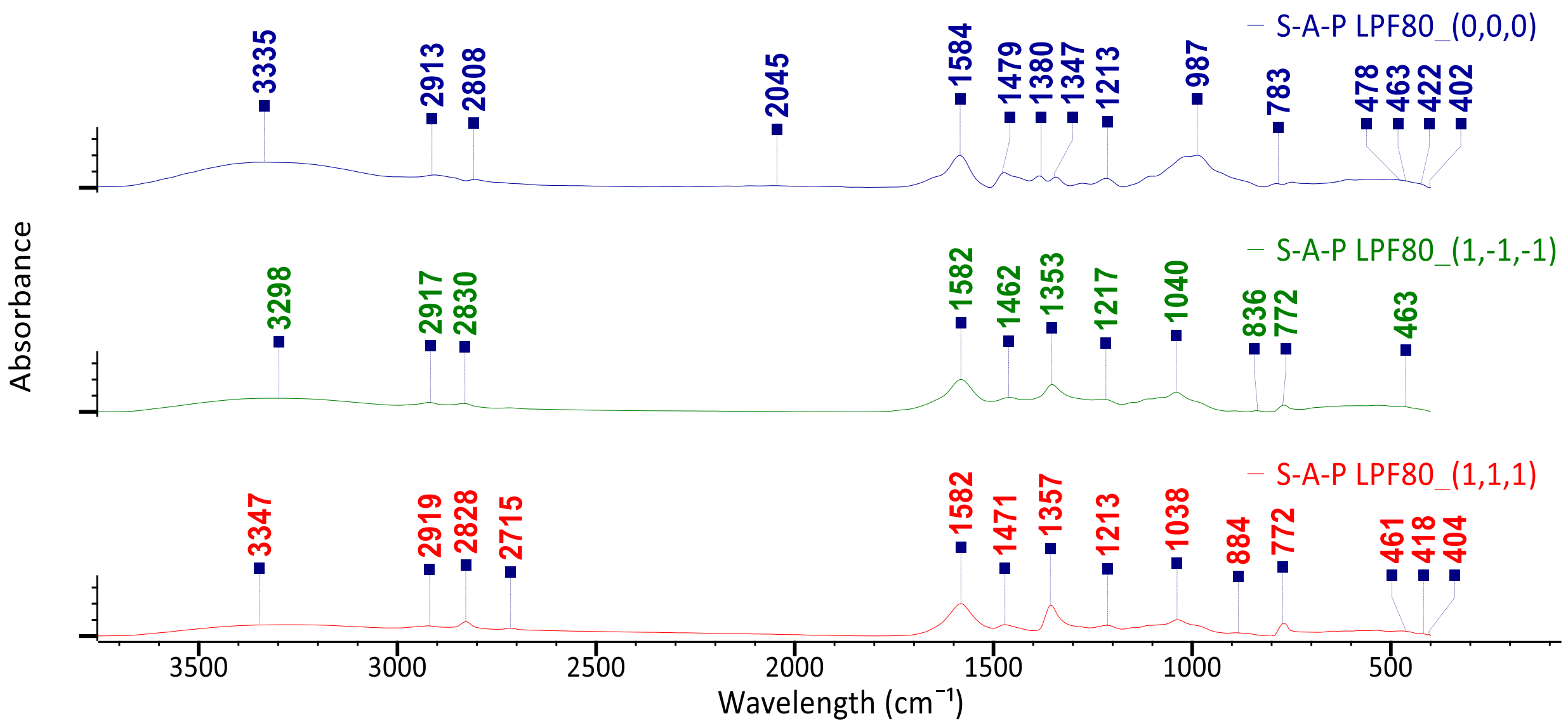

Figure 32. Mid-IR spectra of the S-A-P LPF80 resins of the centre point, highest shear, and lowest shear samples. 


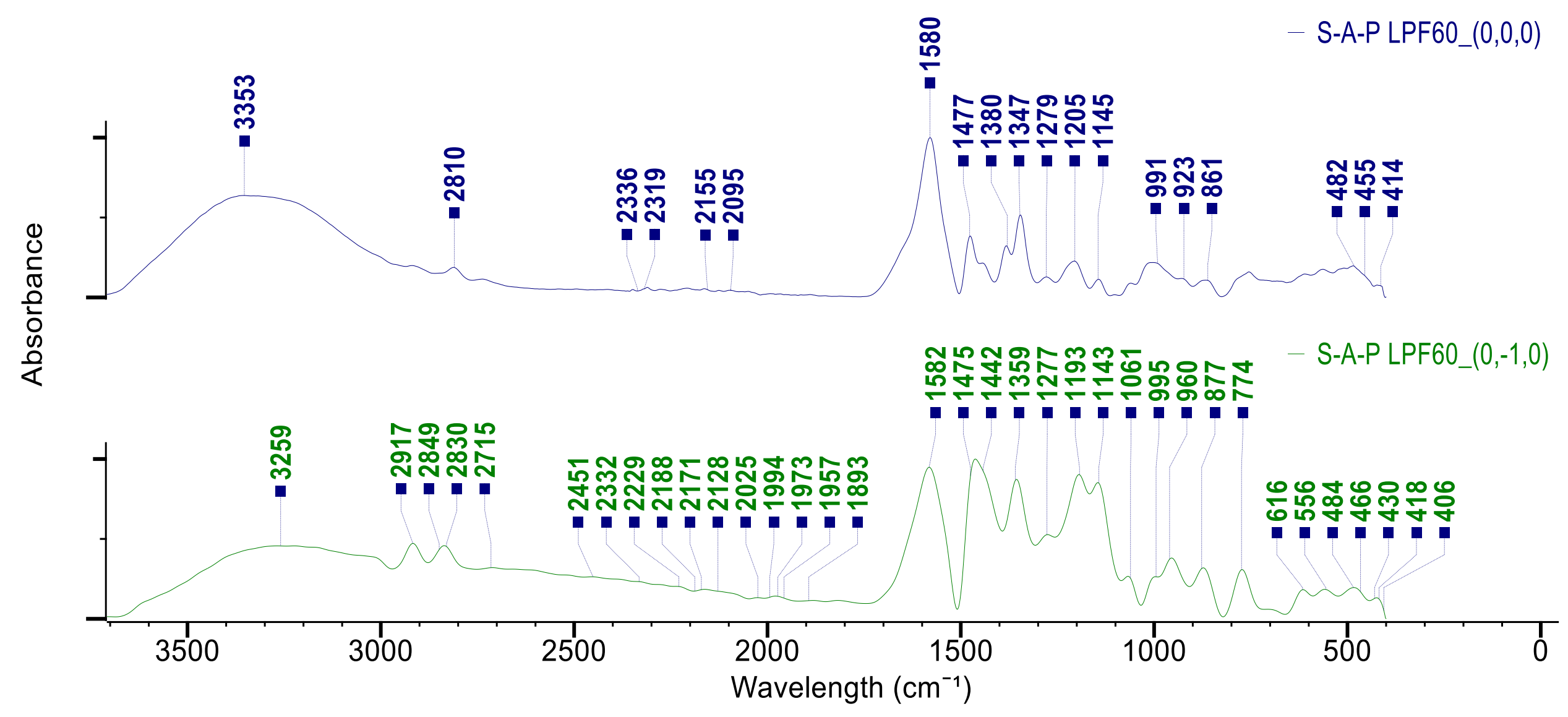

Figure 33. Mid-IR spectra of the S-A-P LPF60 resins of the centre point and highest shear samples. 


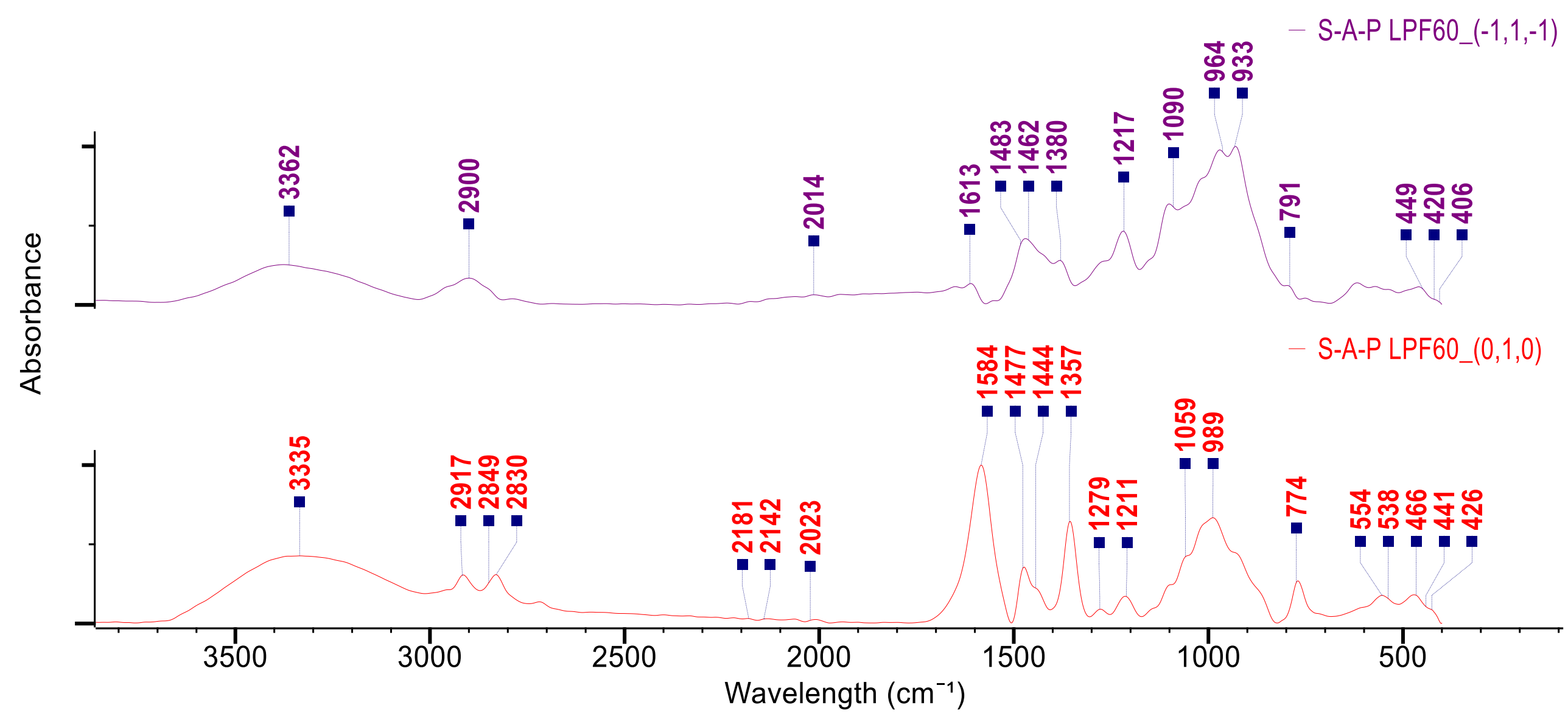

Figure 34. Mid-IR spectra of the S-A-P LPF60 resins with low shear strengths. 


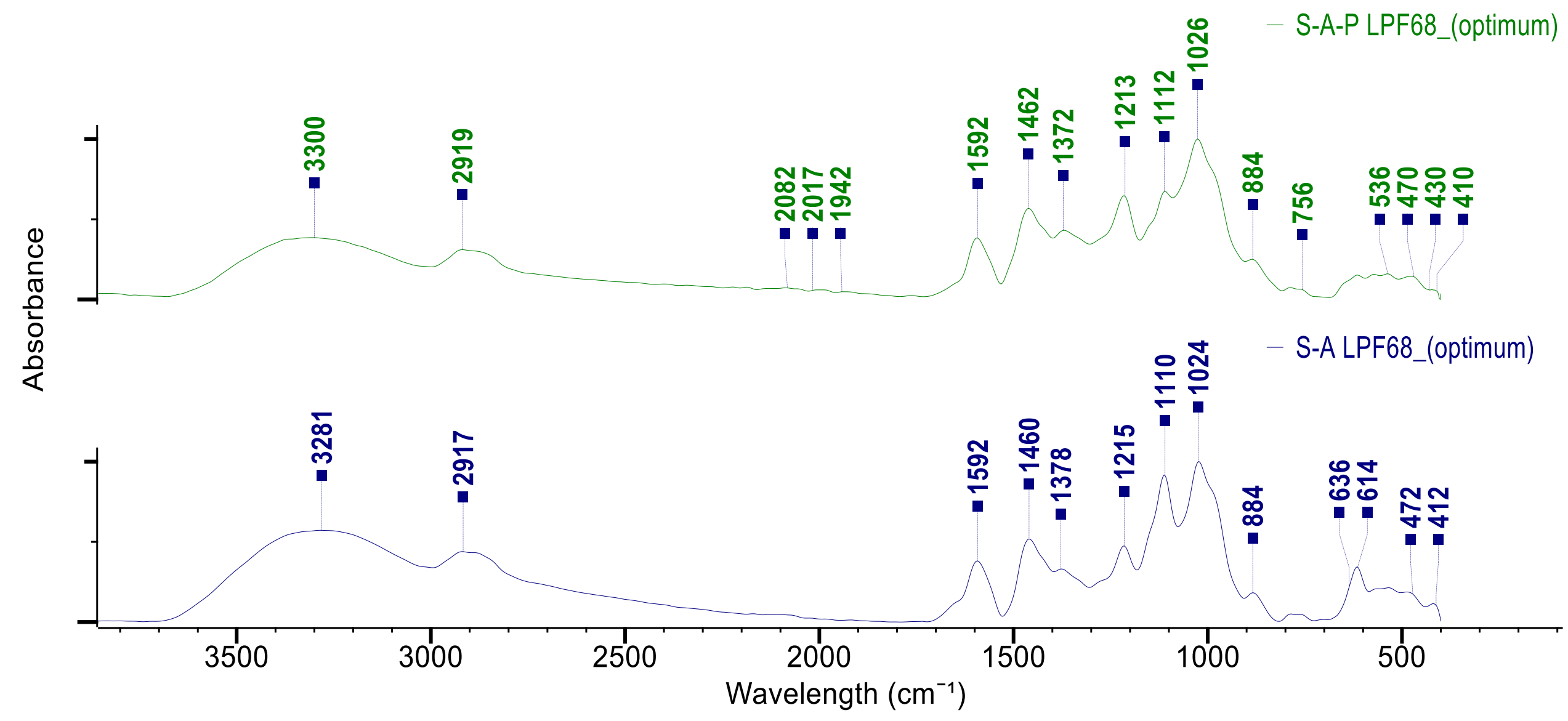

Figure 35. Mid-IR spectra of the S-A and S-A-P LPF68 resins at optimum conditions. 